\title{
OKAMOTO'S SPACE FOR THE FIRST PAINLEVÉ EQUATION IN BOUTROUX COORDINATES
}

\author{
J.J. DUISTERMAAT AND N. JOSHI
}

\begin{abstract}
We study the completeness and connectedness of asymptotic behaviours of solutions of the first Painlevé equation $\mathrm{d}^{2} y / \mathrm{d} x^{2}=6 y^{2}+x$, in the limit $x \rightarrow \infty, x \in \mathbb{C}$. This problem arises in various physical contexts including the critical behaviour near gradient catastrophe for the focusing nonlinear Schrödinger equation. We prove that the complex limit set of solutions is non-empty, compact and invariant under the flow of the limiting autonomous Hamiltonian system, that the infinity set of the vector field is a repellor for the dynamics and obtain new proofs for solutions near the equilibrium points of the autonomous flow. The results rely on a realization of Okamoto's space, i.e., the space of initial values compactified and regularized by embedding in $\mathbb{C P} 2$ through an explicit construction of nine blow-ups.
\end{abstract}

\section{INTRODUCTION}

In this paper, we consider the completeness and connectedness of the asymptotic behaviours of the first Painlevé equation

$$
\frac{\mathrm{d}^{2} y}{\mathrm{~d} x^{2}}=6 y^{2}+x
$$

in the limit $x \rightarrow \infty, x \in \mathbb{C}$. The first Painlevé equation arises in many physical contexts, as a reduction of the Korteweg-de Vries equation, in the double scaling limit of random matrix models and in the critical behaviour near the point of "gradient catastrophe" of the solution to the Cauchy problem for the focusing nonlinear Schrödinger equation [9].

The asymptotic limit $x \rightarrow \infty$ of Equation (1.1) was first studied in 1913 by Boutroux [2], who provided a transformation of variables that makes the asymptotic behaviours explicit. It is known that all solutions of (1.1) are meromorphic in $\mathbb{C}$ with double movable poles, i.e., with locations that change with initial conditions. Boutroux found that locally, in each patch near infinity, the general solutions are given to leading-order by elliptic functions. More detailed results about how the local asymptotic behaviours of solutions change slowly as $x$ moves near infinity were provided by Joshi and Kruskal [15, 16], who constructed a complex multiple-scales method to carry out asymptotic analysis along a large circle in the complex plane for the first and second Painlevé equations. Such local behaviours were used in the Riemann-Hilbert method, which was applied to deduce connections between behaviours valid along special directions approaching infinity (see the review by Kitaev [18]).

2000 Mathematics Subject Classification. 34M55; 58F30; 34C40.

Key words and phrases. Asymptotics, initial-value space, the first Painlevé equation.

Johannes (Hans) Jisse Duistermaat passed away unexpectedly in March 2010. He was affiliated with the Mathematical Institute, University of Utrecht, P.O. Box 80.010, 3508 TA Utrecht, The Netherlands (from 1974 until 2010). Between 2004 and 2010, his research was stimulated by a KNAW professorship.

The collaborative research reported in this paper began at the Isaac Newton Institute for Mathematical Sciences, Cambridge University, UK in May 2009. The first version of this paper was prepared in June 2009. We met again in Leuven, Belgium in July 2009 and continued our collaboration over email. Several successive updates of the paper were prepared by Hans with the last version being dated February 2010. While the research results reported here remain mostly unchanged from that version, minor errors have been corrected and the paper has been restructured to include an abstract and an introduction. Moreover, the detailed explicit calculations of the resolutions of singularities were moved into an appendix to ease the flow of the exposition. Some informal comments, more befitting a working document between two collaborators, have been removed. Nalini Joshi wishes to record her deep gratitude to Hans for his remarkable, revelatory, collaborative friendship. Her research was supported by the Australian Research Council grant \# DP0985615. 
In addition to the two-parameter solutions asymptotic to elliptic-function behaviours, Boutroux identified five one-parameter family of solutions asymptotic to algebraic power expansions in certain sectors of angle $4 \pi / 5$ in $\mathbb{C}$. He called them tronquée or truncated solutions. In each family of tronquée solutions, there is a unique solution whose algebraic expansion is valid in a sector of angle $8 \pi / 5$. Boutroux called these tritronquée or triply truncated solutions. In the literature, this term has come to be associated with the unique tritronquée solution that is real on the real line; each of the other four such solutions can be obtained from this one by a discrete symmetry of Equation (1.1) corresponding to rotating variables in $\mathbb{C}$. This real tritronquée solution appears as a distinguished solution in various physical problems (see, e.g., Dubrovin et al. 9]). In the form $Y_{t t}=6 Y^{2}-t$ (for $y(x)=Y(-t)$ ), Joshi and Kitaev [14] constructed a sequence of solutions that converge to the tritronquée solution on the whole positive real axis and proved that the tritronquée solution has no poles whatsoever on the positive real axis. O. and R. Costin [7] applied Borel-summation methods to deduce many complex properties of the tronquée solutions.

However, while it is known that the solution space of Equation (1.1) is connected, through Okamoto's 21] compactification and regularization of the space of initial-values, there has been no investigation (to our knowledge) of the completeness or connectedness of the known asymptotic behaviours of the solutions of this equation (or of any of the six Painlevé equations). We tackle this problem by undertaking asymptotic analysis in Okamoto's space. Our approach relies on explicit resolution of singularities in an asymptotic version of this space. We note that although we focus on the first Painlevé equation in this paper, our approach can be extended to the other Painlevé equations.

1.1. Outline of the paper. The paper is organized as follows. In $₫ 2$, we recast Equation (1.1) as a Hamiltonian system, provide a rescaling of it under Boutroux's transformation of variables, and summarize known properties of solutions. The resolution of singularities of this BoutrouxPainlevé system is provided explicitly in Appendix A where we carry out the sequence of changes of variables necessary first to compactify and then blow up the nine base points of the system in $\mathbb{C P} 2$. The construction shows that the vector field is infinite on the union $I:=\cup_{i=0}^{8} L_{j}$ of nine complex projective lines.

We show in $\S 3$ that the Boutroux-Painlevé vector field is regular and transverse to the last complex projective line, $L_{9}$, in $S_{9}$. It is shown here that the Taylor expansion of the flow around a point on this line provides us with the Laurent expansion of the solutions $y(x)$ near a pole. In $\$ 4$, we consider the vector field near the infinity set $I$ and show that this is a repellor for the flow. We also construct the limit set for each solution and show that it is a non-empty, compact and connected subset of $S_{9}$, which remains invariant under the autonomous flow. As a corollary, we prove that every solution of Equation (1.1) must have an infinite number of poles in the complex plane. Finally, in $\$ 5$. we consider the Boutroux-Painlevé system near the equilibria of the autonomous limit system and prove several results about tronquée solutions, ending with a determination of their sequence of poles near the boundaries of pole-free sectors, by using classical methods.

1.2. Notation. The Painlevé flow is denoted $\left(y_{1}, y_{2}\right)$, where $y=y_{1}, \mathrm{~d} y / \mathrm{d} x=y_{2}$. In Boutroux's coordinates, this flow is transformed to $\left(u_{1}, u_{2}\right)$, where $u_{1}(z)=x^{-1 / 2} y_{1}(x), u_{2}(z)=x^{-3 / 4} y_{2}(x)$, with $(4 / 5) x^{5 / 4}=z$. We embed the resulting Painlevé-Boutroux flow into $\mathbb{C P} 2$ by identifying the affine coordinates $\left(u_{1}, u_{2}\right)$ with homogeneous coordinates as follows

$$
\begin{aligned}
{\left[1: u_{1}: u_{2}\right] } & =\left[u_{1}^{-1}: 1: u_{1}^{-1} u_{2}\right]=:\left[u_{021}: 1: u_{022}\right] \\
& =\left[u_{2}^{-1}: u_{1} u_{2}^{-1}: 1\right]=:\left[u_{031}: u_{032}: 1\right]
\end{aligned}
$$

The line at infinity given respectively by $u_{021}=0, u_{031}=0$ is denoted by $L_{0}$. For $0 \leq i \leq 8$, corresponding to the $i$-th stage of the regularizing (blow-up) sequence, we denote a base point by $b_{i}$, the exceptional line attached to that base point by $L_{i+1}$ and the coordinates in the $j$-th chart 
of the $i$-th blowup by $\left(u_{i j 1}, u_{i j 2}\right)$. In each coordinate chart, the Jacobian of the coordinate change from $\left(u_{1}, u_{2}\right)$ to $\left(u_{i j 1}, u_{i j 2}\right)$ is denoted by

$$
w_{i j}=\frac{\partial u_{i j 1}}{\partial u_{1}} \frac{\partial u_{i j 2}}{\partial u_{2}}-\frac{\partial u_{i j 1}}{\partial u_{2}} \frac{\partial u_{i j 2}}{\partial u_{1}} .
$$

The last space $S_{9}$ constructed by this sequence of blow-ups is Okamoto's "space of initial values." The regularization of the limiting autonomous system obtained as $z \rightarrow \infty$ is the same as that of the related Weierstrass cubic curve. We add the superscript ell to the above notation for the base points and lines and a superscript 0 to the vector field to refer to the corresponding objects in the resulting anti-canonical pencil.

\section{Boutroux SCALING}

Equation (1.1) can be viewed, upon the substitutions $y=y_{1}, \mathrm{~d} y / \mathrm{d} x=y_{2}$, as a Hamiltonian system $\mathrm{d} y_{1} / \mathrm{d} x=\partial H / \partial y_{2}, \mathrm{~d} y_{2} / \mathrm{d} x=-\partial H / \partial y_{1}$ with an $x$-dependent Hamiltonian function

$$
H=H\left(x, y_{1}, y_{2}\right):=y_{2}^{2} / 2-2 y_{1}^{3}-x y_{1} .
$$

The function $H$ is a weighted homogeneous polynomial in the sense that if we substitute $x=\lambda^{4} \xi$, $y_{1}=\lambda^{2} u_{1}$, and $y_{2}=\lambda^{3} u_{2}$, then $H=\lambda^{6}\left(u_{2}^{2} / 2-2 u_{1}^{3}-\xi u_{1}\right)$. We have $\xi=1$ if and only if $x=\lambda^{4}$, when $\lambda=x^{1 / 4}, y_{1}=x^{1 / 2} u_{1}, u_{1}=x^{-1 / 2} y_{1}, y_{2}=x^{3 / 4} u_{2}$, and $u_{2}=x^{-3 / 4} y_{2}$. If $x=x(z)$ and a dot means differentiation with respect to $z$, then $\dot{u}_{1}=\dot{x}\left(-(1 / 2) x^{-1} u_{1}+x^{1 / 4} u_{2}\right)$. If we choose $(4 / 5) x^{5 / 4}=z$ then $\dot{x} x^{1 / 4}=1$, and the Painlevé system takes the form

$$
\begin{aligned}
& \dot{u}_{1}=u_{2}-2(5 z)^{-1} u_{1}, \\
& \dot{u}_{2}=6 u_{1}^{2}+1-3(5 z)^{-1} u_{2} .
\end{aligned}
$$

This is an order $z^{-1}$ perturbation of the Hamiltonian system with Hamiltonian function equal to the $z$-independent energy function

$$
\begin{aligned}
E & :=u_{2}^{2} / 2-2 u_{1}^{3}-u_{1}, \\
\text { where } \quad \dot{E} & =(5 z)^{-1}\left(2 u_{1}+12 u_{1}^{3}-3 u_{2}^{2}\right)=-(5 z)^{-1}\left(6 E+4 u_{1}\right) .
\end{aligned}
$$

It implies Boutroux's second order differential equation

$$
\ddot{u}_{1}=6 u_{1}^{2}+1-z^{-1} \dot{u}_{1}+4(5 z)^{-2} u_{1}
$$

for $u_{1}$. These transformations have been used by Boutroux [2] in order to investigate the asymptotic behavior of the solutions of the Painlevé equation when $x \rightarrow \infty$.

Because $\int_{z_{0}}^{\infty} z^{-1} \mathrm{~d} z=\infty$, we cannot straightforwardly conclude that solutions of the Boutroux system (2.2) converge to solutions of the autonomous Hamiltonian system when $z \rightarrow \infty$. Actually they don't: we will see that each solution of $(2.2)$ converges to different solutions of the autonomous limit system, depending on the path along which $z$ runs to infinity.

Remark 2.1. If $a$ and $b$ are nonzero complex constant complex numbers, then the substitutions $y(x)=a \eta(\xi), \xi=b x$ turn (1.1) into the differential equation $\mathrm{d}^{2} \eta / \mathrm{d} \xi^{2}=\alpha \eta^{2}+\beta \xi$, where $\alpha=6 a / b^{2}$ and $\beta=1 / a b^{3}$, or equivalently $b=(6 / \alpha \beta)^{1 / 5}$ and $a=\alpha b^{2} / 6$.

Boutroux [2, p. 311] took the first Painlevé equation with the constants $\alpha=6$ and $\beta=-6$, or equivalently $b=(-1 / 6)^{1 / 5}$ and $a=b^{2}$, as his point of departure, and applied the substitutions $X=(4 / 5) \xi^{5 / 4}$ and $\eta=\xi^{1 / 2} Y$ in order to arrive at the differential equation $\mathrm{d}^{2} Y / \mathrm{d} X^{2}=6 Y^{6}-$ $6-(1 / X) \mathrm{d} Y / \mathrm{d} X+\left(4 /(5 X)^{2}\right) Y$. Therefore the translation from Boutroux's notation to ours is $X=(-1 / 6)^{1 / 4} z, Y=(-1 / 6)^{-1 / 2} u=(-1 / 6)^{-1 / 2} u_{1}$, and $Y^{\prime}=(-1 / 6)^{-3 / 4} \dot{u}=(-1 / 6)^{-3 / 4}\left(u_{2}-\right.$ $\left.2(5 z)^{-1} u_{1}\right)$. An expression which plays a central role in Boutroux [2, $\left.\S 7-11\right]$ is

$$
\left(Y^{\prime}\right)^{2}-4 Y^{3}+12 Y=(-1 / 6)^{-3 / 2}\left(\dot{u}^{2}-4 u^{3}-2 u\right) \text {. }
$$


Joshi and Kruskal [15], [16] took the first Painlevé equation with the constants $\alpha=3 / 2$ and $\beta=-3 / 2$, or equivalently $b=(-8 / 3)^{1 / 5}$ and $a=b^{2} / 4$, as their point of departure, and applied the substitutions $Z=(4 / 5) \xi^{5 / 4}$ and $\eta=\xi^{1 / 2} U$, where they actually wrote $z$ and $u$ instead of $Z$ and $U$, respectively. Therefore the translation from their $z$ and $u$ to ours is $Z=(-8 / 3)^{1 / 4} z$, $U=4(-8 / 3)^{-1 / 2} u= \pm \mathrm{i} \sqrt{6} u_{1}$, and $\mathrm{d} U / \mathrm{d} Z=4(-8 / 3)^{-3 / 4} \dot{u}=4(-8 / 3)^{-4 / 3}\left(u_{2}-2(5 z)^{-1} u_{1}\right)$. A central role is played in [15], [16] by the function

$$
\mathcal{E}:=\left((\mathrm{d} U / \mathrm{d} Z)^{2}-U^{3}+3 U\right) / 2=2^{-1 / 2}(-3)^{3 / 2}\left(\dot{u}^{2} / 2-2 u^{3}-u\right) .
$$

The functions $\left(Y^{\prime}\right)^{2}-4 Y^{3}+12 Y$ and $\mathcal{E}$ are closely related to the energy function $E$ in $[2.3$, as

$$
\dot{u}^{2} / 2-2 u^{3}-u=E-2(5 z)^{-1} u_{1} u_{2}+2(5 z)^{-2} u_{1}^{2} .
$$

Remark 2.2. The Boutroux substitutions $x=((5 / 4) z)^{4 / 5}$ with inverse $z=(4 / 5) x^{5 / 4}$, and $y(x)=$ $x^{1 / 2} u(z)=x^{1 / 2} u\left((4 / 5) x^{5 / 4}\right)=((5 / 4) z)^{2 / 5} u(z)$ with inverse $u(z)=x^{-1 / 2} y(x)$ are singular at $x=0$ and correspondingly $z=0$. These substitutions introduce multi-valuedness of the solutions $u(z)$ of the Boutroux-Painlevé equation (2.4) when $z$ runs around the origin in the complex plane, where the solutions $y(x)$ of the Painlevé equation (1.1) are single-valued.

More precisely, every local solution $y(x)$ of (1.1) extends to a single-valued meromorphic function on the whole complex $x$-plane, where the poles are of order two and have leading coefficient equal to 1. This is the Painlevé property in its strongest form; see [10, Remark 1.1] for some remarks on its proofs in the literature.

The equation $u(z)=((5 / 4) z)^{-2 / 5} y\left(((5 / 4) z)^{4 / 5}\right)$, in combination with the single-valuedness of $y(x)$, implies that the analytic continuation of $u(z)$ along the path $z \mathrm{e}^{\mathrm{i} \theta}$, avoiding the poles of $u(z)$, returns to its opposite if $\theta \in \mathbb{R}$ runs from 0 to $5 / 4$ times $2 \pi$. This may be expressed by the formula

$$
u_{1}\left(z e^{5 \pi \mathrm{i} / 2}\right)=-u_{1}(z), \quad u_{2}\left(z e^{5 \pi \mathrm{i} / 2}\right)=\mathrm{i} u_{2}(z),
$$

where the second equation follows from the first, in view of the first equation in (2.2). This observation has been used in Joshi and Kruskal [15, Sec. 5] as a consistency check for their asymptotic results for $u(z)$ for large $|z|$.

Remark 2.3. Each solution $y(x)$ of the Painlevé equation (1.1) has a convergent Laurent expansion

$$
y(x)=\sum_{n=n_{0}}^{\infty} y_{n} x^{n}
$$

for $0<|x|<<1$, when $u(z)=((5 / 4) z)^{-2 / 5} y(x)=((5 / 4) z)^{-2 / 5} y\left(((5 / 4) z)^{4 / 5}\right)$ implies the convergent power series

$$
u(z)=\sum_{n=n_{0}}^{\infty} y_{n}((5 / 4) z)^{(-2+4 n) / 5}
$$

for $0<|z|<<1$. We have the following cases.

i) $y(0)$ is finite, when $n_{0}=0$. The Painlevé equation $y^{\prime \prime}=6 y^{2}+x$ is equivalent to the recursive equations

$$
\begin{aligned}
n(n-1) y_{n} & =6 \sum_{m=0}^{n-2} y_{n-2-m} y_{m} \text { for } n \geq 2, n \neq 3, \\
y_{3} & =2 y_{0} y_{1}+\underset{4}{1 / 6}
\end{aligned}
$$


for the coefficients $y_{n}$. The mapping which assigns to the solution $y(x)$ the complex numbers $y_{0}=y(0)$ and $y_{1}=y^{\prime}(0)$ is bijective from the set of all regular solutions $y(x)$ near $x=0$ onto $\mathbb{C}^{2}$. Subcases:

ia) $y_{0}=y_{1}=0$, when (2.7) for $n=2$ yields that $y_{2}=0$, whereas 2.8$)$ implies that $y_{3}=1 / 6$. An induction on $n$ yields that $y_{n}=0$ unless $n \in 3+5 \mathbb{Z}$, as $n-2-m=3+5 k$ and $m=3+5 l$ imply that $n=3+5(k+l+1)$. Because $-2+4(3+5 j)=10(2 j+1)$, it follows that $u(z)=\sum_{j=0}^{\infty} y_{3+5 j}((5 / 4) z)^{2(2 j+1)}$. In particular this solution $u(z)$ is single-valued.

ib) $y_{0}=0$ and $y_{1} \neq 0$, when $u(z)=y_{1}((5 / 4) z)^{2 / 5}+\mathrm{O}\left(z^{2}\right)$ as $z \rightarrow 0$.

ic) $y_{0} \neq 0$, when $u(z)=y_{0}((5 / 4) z)^{-2 / 5}+\mathrm{O}\left(z^{2 / 5}\right)$ as $z \rightarrow 0$.

ii) $y(x)$ has a pole at $x=0$, when $n_{0}=-2, y_{-2}=1, y_{-1}=y_{0}=y_{1}=y_{2}=0$, and $y_{3}=-1 / 6$. The mapping which assigns to the solution $y(x)$ the coefficient $y_{4}$ is bijective from the set of solutions with a pole at $x=0$ onto $\mathbb{C}$, see for instance [10, the text following (11.3)]. For $n \geq 5$ the Painlevé equation $y^{\prime \prime}=6 y^{2}+x$ implies the recursive equations

$$
(n(n-1)-12) y_{n}=6 \sum_{m=3}^{n-2} y_{n-2-m} y_{m} \text {. }
$$

Subcases:

iia) $y_{4}=0$. As in ia) it follows from (2.9) by induction on $n$ that $y_{n}=0$ unless $n \in 3+5 \mathbb{Z}$, and it follows that $u(z)=\sum_{j=-1}^{\infty} y_{3+5 j}((5 / 4) z)^{2(2 j+1)}$. In particular this solution $u(z)$ is single-valued.

iib) $y_{4} \neq 0$, when $u(z)=((5 / 4) z)^{-2}-(1 / 6)((5 / 4) z)^{2}+y_{4}((5 / 4) z)^{14 / 5}+\mathrm{O}\left(z^{18 / 5}\right)$ as $z \rightarrow 0$.

It follows that the solution $u(z)$ of the Boutroux-Painlevé equation (2.4) is not single-valued, unless we are in the cases ia) or iia). That is, the solution $y(x)$ of (1.1) is either equal to the unique regular solution near $x=0$ for which $y(0)=y^{\prime}(0)=0$, or $y(x)$ is the unique solution of (1.1) with a pole at $x=0$ such that $y_{4}=0$.

If $y(x)$ is a solution of (1.1), and $a \in \mathbb{C}$ is a fifth root of unity, that is, $a^{5}=1$, then $x \mapsto a^{-1} y\left(a^{2} x\right)$ is also a solution of (1.1). The solutions $y(x)$ in ia) and iia), corresponding to the single-valued solutions $u(z)$ of $(2.4)$, are exactly the solutions which are invariant under this five-fold symmetry, that is, which satisfy $y(x)=a^{-1} y\left(a^{2} x\right)$ for every fifth root of unity $a$, as this means that in the Laurent expansion of $y(x)$ only the powers $x^{j}$ appear such that $2 j-1 \in 5 \mathbb{Z} \Leftrightarrow 2 j-1 \in 5(2 \mathbb{Z}+1)$ $\Leftrightarrow j \in 5 Z+3$. The solutions $y(x)$ in ia) and iia) appear in Boutroux [2, p. 336, 337].

Remark 2.4. If $y(x)$ is a solution of the Painlevé equation (1.1), and $a \in \mathbb{C}$ is a fifth root of unity, that is, $a^{5}=1$, then $x \mapsto a^{-1} y\left(a^{2} x\right)$ is also a solution of (1.1).

The solutions $y(x)$ in ia) and iia) of Remark 2.3. corresponding to the single-valued solutions $u(z)$ of the Boutroux-Painlevé equation (2.4), are exactly the solutions which are invariant under this five-fold symmetry, that is which satisfy $y(x)=a^{-1} y\left(a^{2} x\right)$ for every fifth root of unity $a$, as this means that in the Laurent expansion of $y(x)$ only the powers $x^{j}$ appear such that $2 j-1 \in 5 \mathbb{Z}$ $\Leftrightarrow 2 j-1 \in 5(2 \mathbb{Z}+1) \Leftrightarrow j \in 5 Z+3$. This explains why Boutroux [2, p. 336, 337] called the solutions $y(x)$ in ia) and iia) of Remark 2.3 the symmetric solutions.

\section{The POles in OKAMoto's SPACE}

In this section, we consider the Boutroux-Painlevé vector field in Okamoto's space $S_{9}$ constructed explicitly in Appendix A. This construction shows that the vector field has no base points in $S_{9}$, is infinite along the configuration $I:=\bigcup_{i=0}^{8} L_{i}^{(9-i)}$ of nine complex projective lines, and regular in $S_{9} \backslash I$. For this reason we will call the set $I$ the infinity set of the vector field. 
The change of coordinates to the coordinate chart $\left(u_{911}, u_{912}\right)$ is shown in Appendix A to be

$$
\begin{aligned}
u_{911}(z) & =u_{1}(z)^{-9} u_{2}(z)\left(-32 u_{1}(z)^{7} u_{2}(z)-4 u_{1}(z)^{3} u_{2}(z)^{5}+u_{2}(z)^{7}+256(5 z)^{-1} u_{1}(z)^{8}\right) \\
u_{912}(z) & =u_{1}(z) u_{2}(z)^{-1} \\
u_{1}(z) & =u_{912}(z)^{-2}\left(4+32 u_{912}(z)^{4}+u_{911}(z) u_{912}(z)^{6}-256(5 z)^{-1} u_{912}(z)^{5}\right)^{-1} \\
u_{2}(z) & =u_{912}(z)^{-3}\left(4+32 u_{912}(z)^{4}+u_{911}(z) u_{912}(z)^{6}-256(5 z)^{-1} u_{912}(z)^{5}\right)^{-1}
\end{aligned}
$$

The set of points in $S_{9} \backslash I$ which project to $L_{0}$, the set where $\left(u_{1}, u_{2}\right)$ is infinite, is equal to $L_{9} \backslash I$. Because $L_{9} \cap I=L_{9} \cap L_{8}^{(1)}$ consists of one point, $L_{9} \backslash I$ is isomorphic to the affine complex plane. The regular vector field in $S_{9} \backslash I$ is nonzero at and transversal to $L_{9} \backslash I$. A solution crosses the complex line $L_{9} \backslash L_{8}^{(1)}$ at the time $z=\zeta$, if and only if $u(z)=u_{1}(z)$ becomes infinite as $z \rightarrow \zeta$. The whole set $L_{9} \backslash I$ is visible in the coordinate chart $\left(u_{911}, u_{912}\right)$, where it is the line $u_{912}=0$, parametrized by $u_{911} \in \mathbb{C}$. Because $u(z)=u_{1}(z)$ given by Equation $(3.1)$ is a rational expression in $z, u_{911}(z)$, and $u_{922}(z)$, and the solution $z \mapsto\left(u_{911}(z), u_{912}(z)\right)$ of the regular non-autonomous system is a complex analytic function in a neighborhood of $z=\zeta$ with $u_{912}(\zeta)=0$ and $a:=u_{911}(\zeta) \in \mathbb{C}$, it follows that the solution $z \mapsto u(z)$ of the Boutroux-Painlevé equation is a meromorphic function in a neighborhood of $z=\zeta$, with a pole of order two. For this reason the line $L_{9} \backslash I$ is called the pole line.

Consider the vector field $\left(\dot{u}_{911}, \dot{u}_{912}\right)$ which is given in Section A.10 of Appendix A. We recall it here for ease of reference by the reader:

$$
\begin{aligned}
\dot{u}_{911}= & \left(4+32 u_{912}{ }^{4}+u_{911} u_{912}{ }^{6}-256(5 z)^{-1} u_{912}\right)^{-1} \\
& \times\left[u _ { 9 1 2 } \left(-2^{11}-2^{6} \cdot 5 u_{911} u_{912}{ }^{2}+2^{13} \cdot 7 u_{912}{ }^{4}-3^{2} u_{911}{ }^{2} u_{912}{ }^{4}\right.\right. \\
& +2^{12} u_{911} u_{912}{ }^{6}+2^{16} \cdot 3 u_{912^{8}}+2^{3} \cdot 3^{2} u_{911}{ }^{2} u_{912}{ }^{8} \\
& \left.+2^{12} \cdot 5 u_{911} u_{912}{ }^{10}+2^{6} \cdot 11 u_{911}{ }^{2} u_{912}{ }^{12}+2^{3} u_{911}{ }^{3} u_{912}{ }^{14}\right) \\
& -2(5 z)^{-1}\left(2^{2} \cdot 3 u_{911}-2^{12} \cdot 3^{2} u_{912}{ }^{2}-2^{5} \cdot 3^{2} \cdot 7 u_{911} u_{912}{ }^{4}\right. \\
& +2^{15} \cdot 3 \cdot 5 u_{912}{ }^{6}+3 u_{911^{2}} u_{912}{ }^{6}+2^{10} \cdot 17 u_{911} u_{912}{ }^{8} \\
& \left.+2^{17} \cdot 19 u_{912}{ }^{10}+2^{13} \cdot 3 \cdot 7 u_{911} u_{912}{ }^{12}+2^{7} \cdot 23 u_{911} u_{912}{ }^{14}\right) \\
& +2^{9}(5 z)^{-2} u_{912}{ }^{3}\left(-2^{6} \cdot 3 \cdot 5+3 u_{911} u_{912}{ }^{2}\right. \\
& \left.+2^{13} u_{912}{ }^{4}+2^{14} \cdot 5 u_{912}{ }^{8}+2^{8} \cdot 11 u_{911} u_{912}{ }^{10}\right) \\
& \left.-2^{24} \cdot 7(5 z)^{-3} u_{912}{ }^{12}\right], \\
= & -\left(4+32 u_{912}{ }^{4}+u_{911} u_{912}{ }^{6}-256(5 z)^{-1} u_{912}\right)^{-1} \\
& \times\left[2-2^{4} u_{912}{ }^{4}-u_{911} u_{912}{ }^{6}+2^{8} u_{912}{ }^{8}+2^{3} u_{911} u_{912}{ }^{10}\right. \\
& +2^{10} u_{912}{ }^{12}+2^{6} u_{911} u_{912}{ }^{14}+u_{911}{ }^{2} u_{912}{ }^{16} \\
& -(5 z)^{-1} u_{912}\left(2^{2}-2^{5} \cdot 7 u_{912}{ }^{4}+u_{911} u_{912}{ }^{6}\right. \\
& \left.+2^{11} u_{912}{ }^{8}+2^{14} u_{912}{ }^{12}+2^{9} u_{911} u_{912}{ }^{14}\right) \\
& \left.+2^{8}(5 z)^{-2} u_{912}{ }^{6}\left(1+2^{8} u_{912}{ }^{8}\right)\right] .
\end{aligned}
$$

It follows from the equation for $\dot{u}_{912}$ that the coefficients of $(z-\zeta)^{i}$ in the Taylor expansion at $z=\zeta$ of $u_{912}(z)$ do not depend on $a$ for $1 \leq i \leq 6$, when 3.1 shows that the coefficients of $(z-\zeta)^{j}$ in the Laurent expansion at $z=\zeta$ of $u(z)$ do not depend on $a$ for $-2 \leq j \leq 3$. Substitution of the Taylor expansion at $z=\zeta$ of order $i$ of $u_{912}(z)$ in the formula for $\dot{u}_{912}$ yields the Taylor expansion at $z=\zeta$ of order $i$ of $\dot{u}_{912}(z)$ of order $i$, hence the Taylor expansion at $z=\zeta$ of order $i+1$ of $u_{912}(z)$, as long as $i \leq 5$. Then substitution of $u_{911}(\zeta)=a$ in the formula for $\dot{u}_{912}$ yields the coefficient of $(z-\zeta)^{6}$ in the Taylor expansion at $z=\zeta$ of $\dot{u}_{912}(z)$, hence of $(z-\zeta)^{7}$ in the Taylor expansion at $z=\zeta$ of $u_{912}(z)$, when (3.1) yields the coefficients of $(z-\zeta)^{j}$ in the Laurent expansion at $z=\zeta$ of 
$u(z)$ for $-2 \leq j \leq 4$. This yields

$$
\begin{aligned}
u_{911}(z)= & a+\mathrm{O}(z-\zeta), \\
u_{912}(z)= & -\frac{1}{2}(z-\zeta)-\frac{1}{2^{2} \cdot 5 \cdot \zeta}(z-\zeta)^{2}+\frac{3}{2^{2} \cdot 5^{2} \cdot \zeta^{2}}(z-\zeta)^{3}-\frac{3 \cdot 7}{2^{3} \cdot 5^{3} \cdot \zeta^{3}}(z-\zeta)^{4} \\
& +\left(\frac{3 \cdot 7 \cdot 19}{2^{3} \cdot 5^{5} \cdot \zeta^{4}}+\frac{1}{2 \cdot 5}\right)(z-\zeta)^{5}-\left(\frac{3 \cdot 7 \cdot 19}{2 \cdot 5^{6} \cdot \zeta^{5}}-\frac{41}{2^{2} \cdot 3 \cdot 5^{2} \cdot \zeta}\right)(z-\zeta)^{6} \\
& +\left(\frac{3 \cdot 19 \cdot 29}{2 \cdot 5^{7} \cdot \zeta^{6}}-\frac{41}{2 \cdot 3 \cdot 5^{3} \cdot \zeta^{2}}+\frac{3 a}{2^{9} \cdot 7}\right)(z-\zeta)^{7}+\mathrm{O}\left((z-\zeta)^{8}\right), \\
u(z)= & (z-\zeta)^{-2}-\frac{1}{5 \cdot \zeta}(z-\zeta)^{-1}+\frac{3}{2^{2} \cdot 5 \cdot \zeta^{2}}-\frac{31}{2 \cdot 5^{3} \cdot \zeta^{3}}(z-\zeta) \\
& +\left(\frac{19 \cdot 283}{2^{4} \cdot 5^{5} \cdot \zeta^{4}}-\frac{1}{2 \cdot 5}\right)(z-\zeta)^{2}-\left(\frac{3 \cdot 11 \cdot 727}{2^{4} \cdot 5^{6} \cdot \zeta^{5}}+\frac{11}{2 \cdot 4 \cdot 5^{2} \cdot \zeta}\right)(z-\zeta)^{3} \\
& +\left(\frac{197 \cdot 443}{2^{6} \cdot 5^{6} \cdot \zeta^{6}}+\frac{29}{2^{3} \cdot 3 \cdot 5^{2} \cdot \zeta^{2}}-\frac{a}{2^{8} \cdot 7}\right)(z-\zeta)^{4}+\mathrm{O}\left((z-\zeta)^{5}\right) .
\end{aligned}
$$

The anticanonical pencil has a base point at the point ${ }^{(1)} b_{8}^{\text {ell }}$ determined by the equations $u_{921}=$ $256(5 z)^{-1}, u_{922}=0$ which is the lift to $S_{9}$ of the point $b_{8}^{\text {ell }}$ of the anticanonical pencil in $S_{8}$. The blowing up of $S_{8}$ in the point $b_{8}$, which is not the base point of the anticanonical pencil, causes $E w_{92}$ to be infinite along $L_{9} \backslash L_{8}^{(1)}$, the line determined by the equation $u_{921}=0$. In turn this forces the energy function $E(z)$ to have a pole at the point $z=\zeta$ where the solution $u(z)$ of the Boutroux-Painlevé equation has a pole.

The equations for $w_{91}, E w_{91}$, and $\dot{E} w_{91}$ (given in Section A.10 imply in combination with the Taylor expansion for $u_{911}(z)$ and $u_{912}(z)$ in $(3.2)$ that $E=-2^{2}(5 \zeta)^{-1}(z-\zeta)^{-1}+2^{-7} a-$ $22(5 \zeta)^{-2}+\mathrm{O}(z-\zeta)$ and $\dot{E}=-2^{2}(5 \zeta)^{-1}(z-\zeta)^{-2}\left(1+\mathrm{O}\left((z-\zeta)^{2}\right)\right)$. Combination of these asymptotic expansions for $E$ and $\dot{E}$ leads to

$$
E(z)=-2^{2}(5 \zeta)^{-1}(z-\zeta)^{-1}+2^{-7} a-22(5 \zeta)^{-2}+\mathrm{O}((z-\zeta) / \zeta),
$$

where the remainder term is uniform for bounded $\zeta^{-1}$ and $a$. It follows that the energy $E(z)$, although it has a pole of order one at $z=\zeta$, is close to $2^{-7} a$ if $|z-\zeta|$ is large compared to $1 /|\zeta|$, and $E(z)=2^{-7} a+\mathrm{O}\left(\zeta^{-1}\right)$ if $z-\zeta$ and $(z-\zeta)^{-1}$ are bounded. That is, for large $|z|, E(z)$ is well approximated by $2^{-7} a$ as soon as $z$ leaves the disc centered at $z=\zeta$ with radius of small order $1 /|\zeta|$, where the approximation improves when $|z-\zeta|$ increases to order one.

\section{The solutions near the Set Where the VeCtor Field is INFinite}

In this section, we consider the vector field near the infinity set $I$ and show that it is repelling for the flow. We also construct the limit set for each solution and show that it is a non-empty, compact and connected subset of $S_{9}$ that remains invariant under the autonomous flow. As a corollary, we prove that every solution of Equation (1.1) must have an infinite number of poles in the complex plane.

Let $\mathcal{S}$ denote the fiber bundle of the surfaces $S_{9}=S_{9}(z), z \in \mathbb{C} \backslash\{0\}$, in which the time-dependent Painlevé vector field $v_{z}$, in the Boutroux scaling, defines a regular (= holomorphic) one-dimensional vector subbundle $\mathcal{P}$ of $\mathcal{S}$. For each $z \in \mathbb{C} \backslash\{0\}$, let $I(z):=\bigcup_{i=0}^{8} L_{i}^{(9-i)}(z)$ be the infinity set, the set of all points in $S_{9}(z)$ where $v_{z}$ is infinite, that is, where $\mathcal{P}$ is "vertical" (or tangent to the fiber). If $\mathcal{I}$ denotes the union in $\mathcal{S}$ of all $I(z), z \in \mathbb{C} \backslash\{0\}$, then $\mathcal{S} \backslash \mathcal{I}$ is Okamoto's "space of initial conditions", fibered by the surfaces $S_{9}(z) \backslash I(z)$, the open subset of $\mathcal{S}$ of all points in $\mathcal{S}$ where $\mathcal{P}$ is transversal to the fibers, and therefore defines a regular infinitesimal connection in the bundle of the $S_{9}(z) \backslash I(z), z \in \mathbb{C} \backslash\{0\}$. Instead of using the coordinate-invariant description of a bundle of 
surfaces with a connection, we will analyse the asymptotic behavior, for $|z| \rightarrow \infty$, of the solutions of the Painlevé equation in the Boutroux scaling, by studying the $z$-dependent vector field in the coordinate systems introduced in Section 2. The solution curve in $\mathcal{S}$ will we denoted by $\gamma=\gamma(z)$, whereas the corresponding solution of the Boutroux-Painlevé differential equation is denoted by $u(z)$. Note that $u(z)$ is equal to the first coordinate $u_{1}(z)$ of $\gamma(z)$ in the $\left(u_{1}, u_{2}\right)$ coordinate system, the 01-coordinate system.

In this section we begin with an asymptotic description of the solutions near the locus $\mathcal{I}$ where the vector field is infinite. In the notation we often drop the dependence on $z$ of the surfaces $S_{9}(z)$. All order estimates will be uniform in $z$ for $z$ bounded away from zero. Near the part $I \backslash L_{8}^{(1)}=\bigcup_{i=0}^{6} L_{i}^{(9-i)} \cup\left(L_{7}^{(2)} \backslash L_{8}^{(1)}\right)$ of $I$ we will use the function $1 / E$, where $E$ is the energy, as an indicator for the distance to $I$, whereas near the remaining part $L_{8}^{(1)}$ of $I$ we switch to $w_{92}$ in the 92 -coordinate system. See the first statement in Lemma 4.2 . The function $1 / E$ is no longer useful as an indicator function near $L_{8}^{(1)}$ because $L_{8}^{(1)}$ contains the lift $\left(b_{8}^{\text {ell }}\right)^{(1)}$ to $S_{9}$ of the base point $b_{8}^{\text {ell }}$ of the anticanonical pencil, and $E$ takes all finite values near $\left(b_{8}^{\text {ell }}\right)^{(1)}$. One of the points of the proof is that $w_{92}$ is approximately constant when the solution runs closely along $L_{8}^{(1)}$.

Lemma 4.1. Let

$$
I^{6}:=\bigcup_{i=0}^{6} L_{i}^{(9-i)} .
$$

For every $\epsilon>0$ there exists a neighborhood $U$ of $I^{6}$ in $S_{9}$ such that $|(\dot{E} / E) /(-6 / 5 z)-1|<\epsilon$ in $U$ and for all $z \in \mathbb{C} \backslash\{0\}$. For every compact subset $K$ of $L_{7}^{(2)} \backslash L_{8}^{(1)}$ there exists a neighborhood $V$ of $K$ in $S_{9}$ and a constant $C>0$ such that $|(\dot{E} / E) z| \leq C$ in $V$ and for all $z \in \mathbb{C} \backslash\{0\}$.

Proof. Because $I^{6}$ is compact, it suffices to prove that every point of it has a neighborhood in $S_{9}$ in which the estimate holds. The quantity $r:=(5 z(\dot{E} / E)+6) / 8=-2 u_{1} / E$ is equal to

$$
\begin{aligned}
& r_{02}=u_{021}^{2} /\left(4+2 u_{021}{ }^{2}-u_{021} u_{022}{ }^{2}\right), \\
& r_{03}=u_{031}{ }^{2} u_{032} /\left(-u_{031}+2 u_{031}{ }^{2} u_{032}+4 u_{032}{ }^{3}\right) \text {, } \\
& r_{11}=u_{111}^{2} u_{112}{ }^{2} /\left(-u_{111}+4 u_{112}{ }^{2}+2 u_{111}{ }^{2} u_{112}{ }^{2}\right) \text {, } \\
& r_{12}=u_{121}{ }^{2} u_{122} /\left(-1+2 u_{121}{ }^{2} u_{122}+4 u_{121}{ }^{2} u_{122}{ }^{3}\right) \text {, } \\
& r_{21}=u_{211}{ }^{2} u_{212}{ }^{3} /\left(-u_{211}+4 u_{212}+2 u_{211}{ }^{2} u_{212}{ }^{3}\right) \text {, } \\
& r_{22}=u_{221}{ }^{3} u_{222}{ }^{2} /\left(-1+4 u_{221} u_{222}{ }^{2}+2 u_{221}{ }^{3} u_{222}{ }^{2}\right) \text {, } \\
& r_{31}=u_{311^{2}} u_{312}{ }^{4} /\left(4-u_{311}+2 u_{311}{ }^{2} u_{312}{ }^{4}\right) \text {, } \\
& r_{32}=u_{321}{ }^{4} u_{322}{ }^{3} /\left(-1+4 u_{322}+2 u_{321}{ }^{4} u_{322}{ }^{3}\right), \\
& r_{41}=u_{412}{ }^{3}\left(4+u_{411} u_{412}\right)^{2} /\left(-u_{411}+2 u_{412}{ }^{3}\left(4+u_{411} u_{412}\right)^{2}\right) \text {, } \\
& r_{42}=u_{421}{ }^{3}\left(4+u_{421}\right)^{2} u_{422}{ }^{4} /\left(-1+2 u_{421}{ }^{3}\left(4+u_{421}\right)^{2} u_{422}{ }^{4}\right) \text {, } \\
& r_{51}=u_{512}{ }^{2}\left(4+u_{511} u_{512}{ }^{2}\right)^{2} /\left(-u_{511}+2 u_{512}{ }^{2}\left(4+u_{511} u_{512}\right)^{2}\right) \text {, } \\
& r_{52}=u_{521}{ }^{2} u_{522}{ }^{3}\left(4+u_{521}{ }^{2} u_{522}\right)^{2} /\left(-1+2 u_{521}{ }^{2} u_{522}{ }^{3}\left(4+u_{521}{ }^{2} u_{522}\right)^{2}\right) \text {, } \\
& r_{61}=u_{612}\left(4+u_{611} u_{612}\right)^{2} /\left(-u_{611}+2 u_{612}\left(4+u_{611} u_{612}{ }^{3}\right)^{2}\right), \\
& r_{62}=u_{621} u_{622}{ }^{2}\left(4+u_{621}{ }^{3} u_{622}{ }^{2}\right)^{2} /\left(-1+2 u_{612} u_{622}{ }^{2}\left(4+u_{621}{ }^{3} u_{622}{ }^{2}\right)^{2}\right) \text {, } \\
& r_{71}=\left(4+u_{711} u_{712}\right)^{2} /\left(-u_{711}+2\left(4+u_{711} u_{712}\right)^{2}\right), \\
& r_{72}=u_{722}\left(4+u_{721}{ }^{4} u_{722}{ }^{3}\right)^{2} /\left(-1+2 u_{722}\left(4+u_{721}{ }^{4} u_{722}{ }^{3}\right)^{2}\right)
\end{aligned}
$$

in the coordinate charts which cover $I^{6}$. The part $L_{0}^{(9)} \backslash L_{3}^{(6)}$ of $I^{6}$ is equal to the line $u_{021}=0$ on which $r_{02}=0$. The part $L_{1}^{(8)} \backslash L_{2}^{(7)}$ of $I^{6}$ is equal to the line $u_{121}=0$ on which $r_{12}=0$. The part 
$L_{2}^{(7)} \backslash L_{3}^{(6)}$ of $I^{6}$ is equal to the line $u_{221}=0$ on which $r_{22}=0$. The part $L_{3}^{(6)} \backslash\left(L_{4}^{(5)} \cup L_{2}^{(1)}\right)$ of $I^{6}$ is equal to the part $u_{311} \neq 4$ on the line $u_{312}=0$ on which $r_{31}=0$. The part $L_{3}^{(6)} \backslash\left(L_{4}^{(5)} \cup L_{0}^{(9)}\right)$ of $I^{6}$ is equal to the part $u_{322} \neq 1 / 4$ on the line $u_{321}=0$ on which $r_{32}=0$. The part $L_{4}^{(5)} \backslash L_{5}^{(4)}$ of $I^{6}$ is equal to the line $u_{421}=0$ on which $r_{42}=0$. The part $L_{5}^{(4)} \backslash L_{6}^{(3)}$ of $I^{6}$ is equal to the line $u_{521}=0$ on which $r_{52}=0$. The part $L_{6}^{(3)} \backslash L_{7}^{(2)}$ of $I^{6}$ is equal to the line $u_{621}=0$ on which $r_{62}=0$. The part $L_{6}^{(3)} \backslash L_{5}^{(4)}$ of $I^{6}$ is equal to the line $u_{722}=0$ on which $r_{72}=0$. This covers all of $I^{6}$, and the proof of the first statement in the lemma is complete.

For the second statement we observe that $L_{7}^{(2)} \backslash\left(L_{6}^{(3)} \cup L_{8}^{(1)}\right)$ is the line $u_{712}=0, u_{711} \neq 32$ on which $r_{71}=16 /\left(u_{711}-32\right)^{2}$, whereas $u_{721}=0, u_{722} \neq 1 / 32$, on which $r_{72}=16 u_{722} /\left(32 u_{722}-1\right)^{2}$, is an open neighborhood of $L_{7}^{(2)} \cap L_{6}^{(3)}$ in $L_{7}^{(2)}$. Note that $r$ becomes infinite when approaching $L_{7}^{(2)} \cap L_{6}^{(3)}$ on $L_{7}^{(2)}$, which is why $L_{7}^{(2)}$ cannot be included in the first statement of the lemma.

The function $|d|$ in the following lemma will be used as a measure for the distance to the infinity set $I$ of the vector field.

Lemma 4.2. Suppose $z$ is bounded away from zero. Let $q:=2 E$. There exists a continuous complex valued function $d$ on a neighborhood of $I$ in $S_{9}$ such that $d=q^{-1}$ in a neighborhood in $S_{9}$ of $I \backslash L_{8}^{(1)}, d=w_{92}$ in a neighborhood in $S_{9}$ of the remaining part $L_{8}^{(1)} \backslash L_{7}^{(2)}$ of $I$, and $q d \rightarrow 1$, $d / w_{92} \rightarrow 1$ when approaching $L_{8}^{(1)} \backslash L_{7}^{(2)}$.

If the solution at the complex time $z$ is sufficiently close to a point of $L_{8}^{(1)} \backslash L_{7}^{(2)}$ (parametrized by coordinate $\left.u_{921}\right)$, then there exists a unique $\zeta \in \mathbb{C}$ such that $|z-\zeta|=\mathrm{O}\left(|d(z)|\left|u_{921}(z)\right|\right)$, where $d(z)$ is small and $\left|u_{921}(z)\right|$ is bounded, and $u_{921}(\zeta)=0$, that is, the solution of the Boutroux-Painlevé equation has a pole at $z=\zeta$. In the sequel we write $\delta:=d(\zeta)=w_{92}(\zeta)=2^{6} u_{922}(\zeta)$, and consider $\delta \rightarrow 0$. We have $d(z) / \delta \sim 1$. For large finite $R_{8} \in \mathbb{R}_{>0}$, the connected component of $\zeta$ in $\mathbb{C}$ of the set of all $z \in \mathbb{C}$ such that $\left|u_{921}(z)\right| \leq R_{8}$ is an approximate disc $D_{8}$ with center at $\zeta$ and radius $\sim 2^{-5}|\delta| R_{8}$, and $z \mapsto u_{921}(z)$ is a complex analytic diffeomorphism from $D_{8}$ onto $\left\{u \in \mathbb{C}|| u \mid \leq R_{8}\right\}$.

For $i$ decreasing from 7 to 4 we use the coordinate $u_{(i+1) 21}$ in order to parametrize $L_{i}^{(9-i)} \backslash$ $L_{i-1}^{(10-i)}$, where $u_{(i+1) 21}=0$ corresponds to the intersection point of $L_{i}^{(9-1)}$ with $L_{i+1}^{(8-i)}$. The point on $L_{i+1}^{(8-i)} \backslash L_{i}^{(9-i)}$ with coordinate $u_{(i+2) 21}$ runs to the same intersection point when $\left|u_{(i+2) 21}\right| \rightarrow \infty$. For large finite $R_{i} \in \mathbb{R}_{>0}$, the connected component of $\zeta$ in $\mathbb{C}$ of the set of all $z \in \mathbb{C}$ such that the solution at the complex time $z$ is close to $L_{i}^{(9-i)} \backslash L_{i-1}^{(10-i)}$, with $\left|u_{(i+1) 21}(z)\right| \leq R_{i}$, but not close to $L_{i+1}^{(8-i)}$, is the complement of $D_{i+1}$ in an approximate disc $D_{i}$ with center at $\zeta$ and radius $\sim\left(2^{3-i}|\delta| R_{i}\right)^{1 /(9-i)}$, where we note that $|\delta|^{1 /(9-i)} /|\delta|^{1 /(9-(i+1))}=|\delta|^{-1 /(9-i)(8-i)}>>1$. More precisely, $z \mapsto u_{(i+1) 21}$ defines a $(9-i)$-fold covering from the annular domain $D_{i} \backslash D_{i+1}$ onto the complement in $\left\{u \in \mathbb{C}|| u \mid \leq R_{i}\right\}$ of an approximate disc with center at the origin and small radius $\sim\left(2^{-6}|\delta| R_{i+1}{ }^{9-i}\right)^{1 /(8-i)}$, where $u_{(i+1) 21}(z) \sim-2^{i-3} \delta(z-\zeta)^{9-i}$.

For all $z \in D_{4}$, the largest approximate disc, we have $|z-\zeta|<<|\zeta|$ and $d(z) / \delta \sim 1$.

Proof. Recall that $L_{8}^{(1)} \backslash L_{7}^{(2)}$ is determined by the equation $u_{922}=0$ and is parametrized by $u_{921} \in \mathbb{C}$. Moreover, $L_{9}$ minus one point not on $L_{8}^{(1)}$ corresponds to $u_{921}=0$ and is parametrized by $u_{922}$. For the study of the solutions near the part $L_{8}^{(1)} \backslash L_{7}^{(2)}$ of $I$, we use the coordinates 
$\left(u_{921}, u_{922}\right)$. Asymptotically for $u_{922} \rightarrow 0$ and bounded $u_{921}, z^{-1}$ we have

$$
\begin{aligned}
\dot{u}_{921} & \sim-2^{-1} u_{922}{ }^{-1} \\
w_{92} & \sim 2^{6} u_{922} \\
\dot{w}_{92} / w_{92} & =6(5 z)^{-1}+\mathrm{O}\left(u_{922}{ }^{2}\right)=6(5 z)^{-1}+\mathrm{O}\left(w_{92}{ }^{2}\right) \\
q w_{92} & \sim 1-2^{8}(5 z)^{-1} u_{921}{ }^{-1} .
\end{aligned}
$$

It follows from 4.3 that, as long as the solution is close to a given large compact subset of $L_{8}^{(1)} \backslash L_{7}^{(2)}, w_{92}(z)=(z / \zeta)^{6 / 5} w_{92}(\zeta)(1+\mathrm{o}(1))$, where $z / \zeta \sim 1$ if and only if $|z-\zeta|<<|\zeta|$. In view of (4.2), in this situation, $u_{922}$ is approximately equal to a small constant, when (4.1) yields that $u_{921}(z) \sim u_{921}(\zeta)-2^{-1} u_{922}{ }^{-1}(z-\zeta)$, and it follows that $u_{921}(z)$, the affine coordinate on $L_{8}^{(1)} \backslash L_{7}^{(2)}$, fills an approximate disc centered at $u_{921}(\zeta)$ with radius $\sim R$ if $z$ runs over an approximate disc centered at $\zeta$ with radius $\sim 2\left|u_{922}\right| R$. Therefore, if $\left|u_{922}(\zeta)\right|<<1 /|\zeta|$, the solution at complex times $z$ in a $D$ centered at $\zeta$ with radius $\sim 2\left|u_{922}\right| R$ has the properties that along it $u_{922}(z) / u_{922}(\zeta) \sim 1$ and that $z \mapsto u_{921}(z)$ is a complex analytic diffeomorphism from $D$ onto an approximate disc centered at $u_{921}(\zeta)$ with radius $\sim R$. If $R$ is sufficiently large, we have $0 \in u_{921}(D)$, that is, the solution of the Boutroux-Painlevé equation has a pole at a unique point in $D$. After having established this fact, we can arrange that $u_{921}(\zeta)=0$, that is, the center $\zeta$ of $D$ is equal to the pole point. As long as $|z-\zeta|<<|\zeta|$, we have $d(z) / d(\zeta) \sim 1$, i.e., $2^{6} u_{922}(z) / \delta \sim w_{92}(z) / \delta \sim 1$ and $u_{921}(z) \sim-2^{-1} u_{922}{ }^{-1}(z-\zeta) \sim-2^{5} \delta^{-1}(z-\zeta)$, where for a large finite $R_{8} \in \mathbb{R}_{>0}$ the equation $\left|u_{921}(z)\right|=R_{8}$ corresponds to $|z-\zeta| \sim 2^{-5}|\delta| R_{8}$, which is still small compared to $|\zeta|$ if $|\delta|$ is sufficently small. It follows that the connected component $D_{8}$ of $\zeta$ of the set of all $z \in \mathbb{C}$ such that $\left|u_{921}(z)\right| \leq R_{8}$ is an approximate disc with center at $\zeta$ and small radius $\sim 2^{-5}|\delta| R_{8}$. More precisely, $z \mapsto u_{921}(z)$ is a complex analytic diffeomorphism from $D_{8}$ onto $\left\{u \in \mathbb{C}|| u \mid \leq R_{8}\right\}$, and $d(z) / \delta \sim 1$ for all $z \in D_{8}$. The function $q(z)$ has a simple pole at $z=\zeta$, but it follows from (4.4) that $q(z) w_{92}(z) \sim 1$ as soon as $1>>\left|z^{-1} u_{921}(z)^{-1}\right| \sim\left|\zeta^{-1} 2 u_{922}(\zeta)(z-\zeta)^{-1}\right|=2^{-5}|\delta| /|\zeta(z-\zeta)|$, that is, when $|z-\zeta|>>2^{-5}|\delta| /|\zeta|$. As the approximate radius of $D_{8}$ is $2^{-5}|\delta| R_{8}>>2^{-5}|\delta| /|\zeta|$ because $R_{8}>1 /|\zeta|$, we have $q(z) w_{92}(z) \sim 1$ for $z \in D_{8} \backslash D_{9}$, where $D_{9}$ is a disc centered at $\zeta$ with small radius compared to the radius of $D_{8}$.

The set $L_{7}^{(2)} \backslash L_{6}^{(3)}$ is visible in the coordinate system $\left(u_{821}, u_{822}\right)$, where it corresponds to the equation $u_{822}=0$ and is parametrized by $u_{821} \in \mathbb{C}$. The set $L_{8}^{(1)}$ minus one point corresponds to $u_{821}=0$ and is parametrized by $u_{822} \in \mathbb{C}$. It follows from the equations which express $\left(u_{811}, u_{812}\right)$ and $\left(u_{821}, u_{822}\right)$ in terms of $\left(u_{711}, u_{712}\right)$ that $u_{822}=1 / u_{811}$. Also, $u_{811}=u_{921}-2^{8}(5 z)^{-1}$ which implies that $u_{921} \rightarrow \infty$ if and only if $u_{822} \rightarrow 0$. That is, the point near $L_{8}^{(1)}$ approaches the intersection point with $L_{7}^{(2)}$, when 4.4 implies that $q w_{92} \rightarrow 1$. Therefore the functions $q^{-1}$ and $w_{92}$ can be glued together by means of a continuous interpolation to a continuous function $d$ as asserted in the lemma.

Asymptotically for $u_{822} \rightarrow 0$ and bounded $u_{821}$ and $z^{-1}$, we have

$$
\begin{aligned}
\dot{u}_{821} & \sim-u_{822}{ }^{-1}, \\
\dot{u}_{822} & \sim 2^{-1} u_{821}{ }^{-1}, \\
w_{82} & \sim 2^{6} u_{821} u_{822}{ }^{2}, \\
q w_{82} & \sim 1, \\
\dot{q} / q=\dot{E} / E & \sim-6(5 z)^{-1}-2^{7}(5 z)^{-1} u_{821}{ }^{-1} .
\end{aligned}
$$


It follows from 4.9 and 4.6 that $\dot{q} / q \sim-6(5 z)^{-1}-2^{8}(5 z)^{-1} \dot{u}_{822}$, hence

$$
\begin{aligned}
\log \left(q\left(z_{1}\right) / q\left(z_{0}\right)\right) \sim & \log \left(\left(z_{1} / z_{0}\right)^{-6 / 5}\right) \\
& -\left(2^{8} / 5\right)\left(z_{1}^{-1} u_{822}\left(z_{1}\right)-z_{0}{ }^{-1} u_{822}\left(z_{0}\right)+\int_{z_{0}}^{z_{1}} z^{-2} u_{822}(z) \mathrm{d} z\right) .
\end{aligned}
$$

Therefore $q\left(z_{1}\right) / q\left(z_{0}\right) \sim 1$, if for all $z$ on the segment from $z_{0}$ to $z_{1}$ we have $\left|z-z_{0}\right|<<\left|z_{0}\right|$ and $\left|u_{822}(z)\right|<<\left|z_{0}\right|$. We choose $z_{0}$ on the boundary of $D_{8}$, when $d\left(z_{0}\right)^{-1} \delta \sim q\left(z_{0}\right) \delta \sim q\left(z_{0}\right) w_{92}\left(z_{0}\right) \sim$ 1 , and $\left|u_{921}\left(z_{0}\right)\right|=R_{8}$ implies that $\left|u_{822}\left(z_{0}\right)\right| \sim R_{8}{ }^{-1}<<1$. Furthermore, (4.7) and 4.8) imply that $\left|u_{821}\left(z_{0}\right)\right| \sim 2^{-6}\left|w_{82}\left(z_{0}\right)\right|\left|u_{822}\left(z_{0}\right)\right|^{-2} \sim 2^{-6}|\delta| R_{8}{ }^{-2}$, which is small when $|\delta|$ is sufficiently small. Because $D_{8}$ is an approximate disc with center at $\zeta$ and small radius $\sim 2^{-5}|\delta| R_{8}$, and $R_{8}>>|\zeta|^{-1}$, we have that $\left|u_{921}(z)\right| \geq R_{8}>>1$ hence $\left|u_{822}(z)\right|<<1$ if $z=\zeta+r\left(z_{0}-\zeta\right), r \geq 1$, and $\left|z-z_{0}\right| /\left|z_{0}\right|=(r-1)\left|1-\zeta / z_{0}\right|<<1$ if $r-1$ is small compared to the large number $1 /\left|1-\zeta / z_{0}\right|$.

Then equations (4.8), 4.7), and $q \sim \delta^{-1}$ yield $u_{822}{ }^{-1} \sim\left(\delta^{-1} 2^{6} u_{821}\right)^{1 / 2}$, which in combination with 4.5 leads to $2 \mathrm{~d}\left(u_{821}{ }^{1 / 2}\right) / \mathrm{d} z=-2^{3} \delta^{-1 / 2}$, hence $u_{821}(z)^{1 / 2} \sim u_{821}\left(z_{0}\right)^{1 / 2}-2^{2} \delta^{-1 / 2}\left(z-z_{0}\right)$, and therefore $u_{821}(z) \sim 2^{4} \delta^{-1}\left(z-z_{0}\right)^{2}$ if $\left|z-z_{0}\right|>>\left|u_{821}\left(z_{0}\right)\right|^{1 / 2}$. For large finite $R_{7} \in \mathbb{R}_{>0}$ the equation $\left|u_{821}(z)\right|=R_{7}$ corresponds to $\left|z-z_{0}\right| \sim\left(2^{-4}|\delta| R_{7}\right)^{1 / 2}$, which is still small compared to $\left|z_{0}\right| \sim|\zeta|$, and therefore $|z-\zeta| \leq\left|z-z_{0}\right|+\left|z_{0}-\zeta\right|<<|\zeta|$. This proves the statements about the behavior of the solution near $L_{7}^{(2)} \backslash L_{6}^{(3)}$.

The statements for $4 \leq i \leq 6$ about the behavior of the solutions near the part $L_{i}^{(9-i)} \backslash L_{i-1}^{(10-i)}$ of $I$ will be proved by induction over decreasing $i$. The set $L_{i}^{(9-i)} \backslash L_{i-1}^{(10-i)}$ is visible in the coordinate system $\left(u_{(i+1) 21}, u_{(i+1) 22}\right)$, where it corresponds to the equation $u_{(i+1) 22}=0$ and is parametrized by $u_{(i+1) 21} \in \mathbb{C}$. The set $L_{i+1}^{(8-i)}$ minus one point corresponds to $u_{(i+1) 21}=0$ and is parametrized by $u_{(i+1) 22} \in \mathbb{C}$. It follows from the equations which express $\left(u_{(i+1) 11}, u_{(i+1) 12}\right)$ and $\left(u_{(i+1) 21}, u_{(i+1) 22}\right)$ in terms of $\left(u_{i 11}, u_{i 12}\right)$ that $u_{(i+1) 22}=1 / u_{(i+1) 11}, u_{711}=u_{821}+32, u_{611}=u_{721}$, and $u_{511}=u_{621}$. This shows that $u_{(i+2) 21} \rightarrow \infty$ if and only if $u_{(i+1) 22} \rightarrow 0$, that is, the point near $L_{i+1}^{(8-i)}$ approaches the intersection point with $L_{i}^{(9-i)}$.

Asymptotically for $u_{(i+1) 22} \rightarrow 0$ and bounded $u_{(i+1) 21}$ and $z^{-1}$, we have

$$
\begin{aligned}
\dot{u}_{(i+1) 21} & \sim-(9-i) 2^{-1} u_{(i+1) 22}{ }^{-1}, \\
w_{(i+1) 2} & \sim 2^{6} u_{(i+1) 21^{8-i}} u_{(i+1) 22}{ }^{9-i}, \\
q w_{(i+1) 2} & \sim 1, \\
\dot{q} / q=\dot{E} / E & \sim-6(5 z)^{-1} .
\end{aligned}
$$

Assume that $\left|u_{(i+2) 21}\left(z_{0}\right)\right|=R_{i+1}>>1$, where the induction hypothesis yields that $\mid z_{0}$ $\zeta|<<| \zeta \mid$ and $1 /\left(q\left(z_{0}\right) \delta\right) \sim d\left(z_{0}\right) / \delta \sim 1$. It follows from 4.11, 4.12, and $\left|u_{(i+1) 22}\left(z_{0}\right)\right| \sim$ $1 /\left|u_{(i+2) 21}\left(z_{0}\right)\right|$, that

$$
\left|u_{(i+1) 21}\left(z_{0}\right)\right|^{8-i} \sim 2^{-6}\left|q\left(z_{0}\right)\right|^{-1}\left|u_{(i+1) 22}\right|^{i-9} \sim 2^{-6}|\delta| R_{i+1}{ }^{9-i},
$$

which is small if $|\delta|$ is sufficiently small.

It follows from 4.13 that $q(z) / \delta \sim q(z) / q\left(z_{0}\right) \sim 1$ along a solution near $L_{i}^{(9-i)} \backslash L_{i-1}^{(10-i)}$, as long as $\left|z-z_{0}\right|<<\left|z_{0}\right|$. Then (4.12) and (4.11) yield

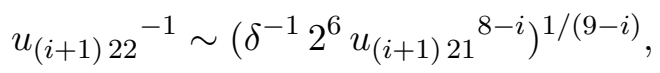

which in combination with 4.10 leads to

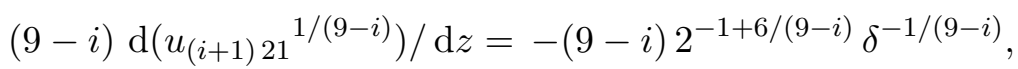


hence

$$
u_{(i+1) 21}(z)^{1 /(9-i)} \sim u_{(i+1) 21}\left(z_{0}\right)^{1 /(9-i)}-2^{(i-3) /(9-i)} \delta^{-1 /(9-i)}\left(z-z_{0}\right),
$$

and therefore $u_{(i+1) 21}(z) \sim 2^{i-3} \delta^{-1}\left(z-z_{0}\right)^{9-i}$ if $\left|z-z_{0}\right|>>\left|u_{(i+1) 21}\left(z_{0}\right)\right|^{1 /(9-i)}$. For large finite $R_{i} \in \mathbb{R}_{>0}$ the equation $\left|u_{(i+1) 21}(z)\right|=R_{i}$ corresponds to $\left|z-z_{0}\right| \sim\left(2^{3-i}|\delta| R_{i}\right)^{1 /(9-i)}$, which is still small compared to $\left|z_{0}\right| \sim|\zeta|$, and therefore $|z-\zeta| \leq\left|z-z_{0}\right|+\left|z_{0}-\zeta\right|<<|\zeta|$.

The following corollary implies that the infinity set $I$ of the vector field is repelling. This in turn implies that every solution which starts in Okamoto's space $S_{9} \backslash I$ remains there for all complex nonzero times.

Corollary 4.3. For every $\epsilon_{1}>0,0<\epsilon_{2}<6 / 5$, and $0<\epsilon_{3}<1$, there exists a $\delta \in \mathbb{R}_{>0}$ such that for every solution we have that if $\left|z_{0}\right| \geq \epsilon_{1}$ and $\left|d\left(z_{0}\right)\right|<\delta$, we have the following conclusions. Let $\rho$ denote the supremum of all $r>\left|z_{0}\right|$ such that $|d(z)|<\delta$ whenever $\left|z_{0}\right| \leq|z| \leq r$. Then

i) $\rho$ is bounded above by the inequality $\delta \geq\left|d\left(z_{0}\right)\right|\left(\rho /\left|z_{0}\right|\right)^{6 / 5-\epsilon_{2}}\left(1-\epsilon_{3}\right)$.

ii) If $\left|z_{0}\right| \leq|z| \leq \rho$, then $d(z)=d\left(z_{0}\right)\left(z / z_{0}\right)^{6 / 5+\varepsilon_{2}(z)}\left(1+\varepsilon_{3}(z)\right)$, where $\left|\varepsilon_{2}(z)\right| \leq \epsilon_{2}$ and $\left|\varepsilon_{3}(z)\right| \leq \epsilon_{3}$.

iii) If $|z| \geq \rho$ then $|d(z)| \geq \delta\left(1-\epsilon_{3}\right)$.

Proof. It follows from Lemma 4.2 that for every solution close to $I$ the set of all not too small complex times $z$ such that the solution is not near $I^{6}$ is a union of approximate discs of radius of order $|d|^{1 / 2}$ where the distance between the discs is at least of order $|d|^{1 / 3}$, where $|d|^{1 / 3} /|d|^{1 / 2}>>1$. Therefore, if the solution is near $I^{6}$ at the complex times $z_{0}$ and $z_{1}$, and is near $I$ for all complex times $z$ such that $|z|$ is between $\left|z_{0}\right|$ and $\left|z_{1}\right|$, there is a path $\gamma$ from $z_{0}$ to $z_{1}$ such that for all $z$ on $\gamma$ we have that the solution at time $z$ is near $I^{6}$, and $\gamma$ is $\mathrm{C}^{1}$ close to the path $[0,1] \ni t \mapsto e^{l_{0}+t\left(l_{1}-l_{0}\right)}$, where $l_{i}=\log z_{i}$. Then Lemma 4.1 implies that $q\left(z_{1}\right)=q\left(z_{0}\right)\left(z_{1} / z_{0}\right)^{-6 / 5+\mathrm{o}(1)}(1+\mathrm{o}(1))$, hence $d(z)=d\left(z_{0}\right)\left(z / z_{0}\right)^{6 / 5+\mathrm{o}(1)}(1+\mathrm{o}(1))$. Because Lemma 4.2 implies that the ratio between the values of $d$ remains close to 1 if the solution stays close to $L_{7}^{(2)} \cup L_{8}^{(1)}$, it follows that $d\left(z_{1}\right)=$ $d\left(z_{0}\right)\left(z_{1} / z_{0}\right)^{6 / 5+\mathrm{o}(1)}(1+\mathrm{o}(1))$ if the solution is close to $I$ at all complex times $z$ such that $|z|$ is between $\left|z_{0}\right|$ and $\left|z_{1}\right|$. The corollary follows from these estimates.

Remark 4.4. The substitutions

$$
\begin{aligned}
& u_{1}(z)=((5 / 4) z)^{-2 / 5} y_{1}\left(((5 / 4) z)^{4 / 5}\right), \\
& u_{2}(z)=((5 / 4) z)^{-3 / 5} y_{2}\left(((5 / 4) z)^{4 / 5}\right)
\end{aligned}
$$

in the beginning of Section 2 lead in combination with $(2.3)$ to

$$
\begin{aligned}
E(z)= & 2^{-1}((5 / 4) z)^{-6 / 5} y_{2}\left(((5 / 4) z)^{4 / 5}\right)^{2} \\
& -2((5 / 4) z)^{-6 / 5} y_{1}\left(((5 / 4) z)^{4 / 5}\right)^{3}-((5 / 4) z)^{-2 / 5} y_{1}\left(((5 / 4) z)^{4 / 5}\right) .
\end{aligned}
$$

Because the solution $\left(y_{1}(x), y_{2}(x)\right)$ of the Painlevé system is single valued, we have the analytic continuation formula

$$
E\left(z \mathrm{e}^{5 \pi \mathrm{i} / 2}\right)=-E(z)
$$

analogous to 2.6 . Because also $\left(z \mathrm{e}^{5 \pi \mathrm{i} / 2}\right)^{-6 / 5}=-z^{-6 / 5}$, the asymptotic formula $E(z) / E\left(z_{0}\right) \sim$ $\left(z / z_{0}\right)^{-6 / 5}$ along solutions close to the part $I \backslash\left(L_{8}^{(1)} \cup L_{7}^{(2)}\right)$ of the inifinity set $I$ is consistent with (4.14.

Because the substitutions of coordinates in Section 2 depend in a polynomial way on $z^{-1}$, the bundle of the complex projective algebraic surfaces $S_{9}(z), z \in \mathbb{C} \backslash\{0\}$ extends to a complex analytic family $\mathcal{S}_{9}=S_{9}(z), z \in \mathbb{P}^{1} \backslash\{0\}$, where the complex projective line $\mathbb{P}^{1}$ is identified with the Riemann sphere $\mathbb{C} \cup\{\infty\}$. The surface $S_{9}(\infty)$ over the point $\infty \in \mathbb{P}^{1}$ is obtained by blowing up $\mathbb{P}^{2}$ nine times as in the definition of $S_{9}(z)$, where in the formulas for the base point $b_{8}(z)$ and the coordinate 
systems $\left(u_{911}, u_{912}\right)$ and $\left(u_{921}, u_{922}\right)$ the coefficient $1 / z$ is replaced by zero. Because $b_{8}(\infty)=b_{8}^{\text {ell }}$, the base point of the anticanonical pencil defined by $w$ and $E w$, the limit surface $S_{9}(\infty)$ is equal to the rational elliptic surface obtained by blowing up the base points of the anticanonical pencil. The Boutroux-Painlevé vector field converges for $z \rightarrow \infty$ to the vector field of the autonomous Hamiltonian system $\dot{u}_{1}=u_{2}, \dot{u}_{2}=6 u_{1}{ }^{2}+1$ with Hamiltonian function equal to the energy function $E$ in 2.3$)$. The function $u_{1}(z)$ satisfies the Weierstrass equation $\left(\dot{u}_{1}\right)^{2}-4 u_{1}^{3}-2 u_{1}=2 E$, which is why in the sequel we will use the function $q:=2 E$ instead of the energy function $E$. The function $q$ defines the elliptic fibration $q: S_{9}(\infty) \rightarrow \mathbb{P}^{1}$, where the fiber $I(\infty)=q^{-1}(\{\infty\})=\lim _{z \rightarrow \infty} I(z)$ over $q=\infty$ is a singular fiber of Kodaira type $\mathrm{II}^{*}$. The vector field of the autonomous Hamiltonian system is regular in the limit fiber $S_{9}(\infty) \backslash I(\infty)$ of Okamoto's space of initial conditions, and infinite on $I(\infty)$. The function $q$ is constant on its solution curves, and each non-singular fiber is an elliptic curve where the time parameter of the solution leads to an identification of the fiber with $\mathbb{C} / P(q)$, where $P(q)$ denotes the period lattice of the flow at the level $q$. The -1 curve $L_{9}(\infty)$ which appears at the last, the ninth blowup is a global holomorphic section for the elliptic fibration. Starting at the complex time $z=0$ on the unique intersection point of the level curve with $L_{9}(\infty)$, the period lattice $P(q)$ is equal to the set of all $z \in \mathbb{C}$ such that the solution of the autonomous Hamiltonian system hits $L_{9}(\infty)$. In view of the Weierstrass equation $\left(\dot{u}_{1}\right)^{2}-4 u_{1}^{3}-2 u_{1}=q$, and the fact that $u_{1}(z)$ has a pole at $z=\zeta$ if and only if $\zeta \in P(q)$, the $u_{1}$-coordinate of this solution is equal to the Weierstrass $\wp$ function of the lattice $P(q)$, and we recover the fact that hitting $L_{9}(\infty)$ corresponds to $u_{1}$ having a pole. The equilibrium points of the autonomous Hamiltonian system are the points in the affine $\left(u_{1}, u_{2}\right)$-charts determined by the equations $u_{2}=0$ and $6 u_{1}^{2}+1=0$. The corresponding singular values are $q=-4 u_{1}(-1 / 6)-2 u_{1}=-(4 / 3) u_{1}= \pm \mathrm{i} \sqrt{8 / 27}$. For each of these two finite singular values of $q$ we have a singular fiber of Kodaira type $\mathrm{I}_{1}$. Therefore the configuration of the singular fibers of the rational elliptic surface $S_{9}(\infty)$ is II $+\mathrm{I}_{1}+\mathrm{I}_{1}$, the second item in Persson's list [23, pp. 7-14] of configurations of singular fibers of rational elliptic surfaces. It also occurs on p. 121 in the classification of Schmickler-Hirzebruch [24] of all elliptic fibrations over $\mathbb{P}^{1}$ with at most three singular fibers.

The following definition is a complex version of the concept of limit sets in dynamical systems.

Definition 4.5. For every solution $\mathbb{C} \backslash\{0\} \ni z \mapsto U(z) \in S_{9}(z) \backslash I(z)$, let $\Omega_{U}$ denote the set of all $s \in S_{9}(\infty) \backslash I(\infty)$ such that there exists a sequence $z_{j} \in \mathbb{C}$ with the property that $z_{j} \rightarrow \infty$ and $U\left(z_{j}\right) \rightarrow s$ as $j \rightarrow \infty$. The subset $\Omega_{U}$ of $S_{9}(\infty) \backslash I(\infty)$ is called the limit set of the solution $U$.

Corollary 4.6 below is analogous to Coddington and Levinson [4, Th. 1.1 and 1.2 in Ch.16].

Corollary 4.6. There exists a compact subset $K$ of $S_{9}(\infty) \backslash I(\infty)$ such that for every solution $U$ the limit set $\Omega_{U}$ is contained in $K$. The limit set $\Omega_{U}$ is a non-empty, compact and connected subset of $K$, invariant under the flow of the autonomous Hamiltonian system on $S_{9}(\infty) \backslash I(\infty)$. For every neighborhood $A$ of $\Omega_{U}$ in $\mathcal{S}_{9}$ there exists an $r>0$ such that $U(z) \in A$ for every $z \in \mathbb{C}$ such that $|z|>r$. If $z_{j}$ is any sequence in $\mathbb{C} \backslash\{0\}$ such that $z_{j} \rightarrow \infty$ as $j \rightarrow \infty$, then there is a subsequence $j=j(k) \rightarrow \infty$ as $k \rightarrow \infty$ and an $s \in \Omega_{U}$ such that $U\left(z_{j(k)}\right) \rightarrow s$ as $k \rightarrow \infty$. Finally, for every solution $U$ the limit set $\Omega_{U}$ is invariant under the transformation $T$ of $S_{9}(\infty)$ which in the coordinate system $\left(u_{1}, u_{2}\right)$ is given by $\left(u_{1}, u_{2}\right) \mapsto\left(-u_{1}\right.$, i $\left.u_{2}\right)$, when $q \mapsto-q$ and $E \mapsto-E$.

Proof. For any $\delta, r \in \mathbb{R}_{>0}$, let $K_{\delta, r}$ denote the set of all $s \in S_{9}(z)$ such that $|z| \geq r$ and $|d(s)| \geq \delta$. Because $\mathcal{S}_{9}$ is a complex analytic family over $\mathbb{P}^{1} \backslash\{0\}$ of compact surfaces $S_{9}(z), z \in \mathbb{P}^{1} \backslash\{0\}, K_{\delta, r}$ is a compact subset $\mathcal{S}_{9}$. Furthermore $K_{\delta, r}$ is disjoint from union of the infinity sets $I(z), z \in \mathbb{P}^{1} \backslash\{0\}$, and therefore $K_{\delta, r}$ is a compact subset of Okamoto's space $\mathcal{S}_{9} \backslash \mathcal{S}_{9, \infty}$, where the latter is viewed as a complex analytic family of non-compact surfaces over $\mathbb{P}^{1} \backslash\{0\}$. When $r \uparrow \infty$, the sets $K_{\delta, r}$ shrink to the set $K_{\delta, \infty}$ of all $s \in S_{9}(\infty)$ such that $|d(s)| \geq \delta$, which is a compact subset of $S_{9}(\infty) \backslash I(\infty)$. 
It follows from Corollary 4.3 that there exists $\delta \in \mathbb{R}_{>0}$ such that for every solution $U$ there exists $r_{0} \in \mathbb{R}_{>0}$ with the property that $U(z) \in K_{\delta, r_{0}}$ for every $z \in \mathbb{C}$ such that $|z| \geq r_{0}$. In the sequel, let $r \geq r_{0}$, when it follows from the definition of $K_{\delta, r}$ that $U(z) \in K_{\delta, r}$ whenever $|z| \geq r$. Let $Z_{r}:=\{z \in \mathbb{C}|| z \mid \geq r\}$ and let $\Omega_{U, r}$ denote the closure of $U\left(Z_{r}\right)$ in $\mathcal{S}_{9}$. Because $Z_{r}$ is connected and $U$ is continuous, $U\left(Z_{r}\right)$ is connected, hence its closure $\Omega_{U, r}$ is connected. Because $U\left(Z_{r}\right)$ is contained in the compact subset $K_{\delta, r}$, its closure $\Omega_{U, r}$ is contained in $K_{\delta, r}$, and therefore $\Omega_{U, r}$ is a non-empty compact and connected subset of $\mathcal{S}_{9} \backslash \mathcal{S}_{9, \infty}$. Because the intersection of a decreasing sequence of non-empty compact and connected sets is non-empty, compact, and connected, and the sets $\Omega_{U, r}$ decrease to $\Omega_{U}$ as $r \uparrow \infty$, it follows that $\Omega_{U}$ is a non-empty, compact and connected subset of $\mathcal{S}_{9}$. Because $\Omega_{U, r} \subset K_{\delta, r}$ for all $r \geq r_{0}$, and the sets $K_{\delta, r}$ shrink to the compact subset $K_{\delta, \infty}$ of $S_{9}(\infty) \backslash I(\infty)$ as $r \uparrow \infty$, it follows that $\Omega_{U} \subset K_{\delta, \infty}$. This proves the first statement in the corollary with $K=K_{\delta, \infty}$. Because $\Omega_{U}$ is the intersection of the decreasing family of compact sets $\Omega_{U, r}$, there exists for every neighborhood $A$ of $\Omega_{U}$ in $\mathcal{S}_{9}$ an $r>0$ such that $\Omega_{U, r} \subset A$, hence $U(z) \in A$ for every $z \in \mathbb{C}$ such that $|z| \geq r$. If $z_{j}$ is any sequence in $\mathbb{C} \backslash\{0\}$ such that $\left|z_{j}\right| \rightarrow \infty$, then the compactness of $K_{\delta, r}$, in combination with $U\left(Z_{r}\right) \subset K_{\delta, r}$, implies that there is a subsequence $j=j(k) \rightarrow \infty$ as $k \rightarrow \infty$ and an $s \in K_{\delta, r}$ such that $U\left(z_{j(k)}\right) \rightarrow s$ as $k \rightarrow \infty$, when it follows from the definition of $\Omega_{U}$ that $s \in \Omega_{U}$.

We next prove that $\Omega_{U}$ is invariant under the flow $\Phi^{t}$ of the autonomous system Hamiltonian system. Let $s \in \Omega_{U}$, when there is a sequence $z_{j} \in \mathbb{C} \backslash\{0\}$ such that $z_{j} \rightarrow \infty$ and $U\left(z_{j}\right) \rightarrow s$ as $j \rightarrow \infty$. Because the $z$-dependent vector field of the Boutroux-Painlevé system converges in $\mathrm{C}^{1}$ to the vector field of the autonomous Hamiltonian system as $z \rightarrow \infty$, it follows from the continuous dependence on initial data and parameters for first order ordinary differential equations, see for instance Coddington and Levinson [4, Th. 7.4 in Ch. 1], that the distance between $U\left(z_{j}+t\right)$ and $\Phi^{t}\left(U\left(z_{j}\right)\right)$ converges to zero as $j \rightarrow \infty$. Because $\Phi^{t}\left(U\left(z_{j}\right)\right) \rightarrow \Phi^{t}(s)$ and $z_{j} \rightarrow \infty$ as $j \rightarrow \infty$, it follows that $U\left(z_{j}+t\right) \rightarrow \Phi^{t}(s)$ and $z_{j}+t \rightarrow \infty$ as $j \rightarrow \infty$, hence $\Phi^{t}(s) \in \Omega_{U}$.

The invariance of $\Omega_{U}$ under the transformation $T$ follows from (2.6) and (2.3).

Corollary 4.7. Every solution of the first Painlevé equation has infinitely many poles.

Proof. Let $u(z)$ be a solution of the Boutroux-Painlevé equation with only finitely many poles, $U(z)$ the corresponding solution of the system in $S_{9} \backslash S_{9, \infty}$, and $\Omega_{U}$ the limit set of $U$. According to Corollary 4.6, $\Omega_{U}$ is a compact subset of $S_{9} \backslash S_{9, \infty}$. If $\Omega_{U}$ intersects the pole line $L_{9}$ in a point $p$, then there exist $z$ with $|z|$ arbitrarily large such that $U(z)$ is arbitrarily close to $p$, when the transversality of the vector field to the pole line implies that $U(\zeta) \in L_{9}$ for a unique $\zeta$ near $z$, which means that $u(z)$ has a pole at $z=\zeta$. As this would imply that $u(z)$ has infinitely many poles, it follows that $\Omega_{U}$ is a compact subset of $S_{9} \backslash\left(S_{9, \infty} \cup L_{9}\right)$. However, $S_{9, \infty} \cup L_{9}$ is equal to the set of all points in $S_{9}$ which lie over the line $L_{0}$ at infinity in the complex projective plane, and therefore $S_{9} \backslash\left(S_{9, \infty} \cup L_{9}\right)$ is the affine $\left(u_{1}, u_{2}\right)$ coordinate chart, of which $\Omega_{U}$ is a compact subset, which implies that $u_{1}(z)=u(z)$ and $u_{2}(z)$ remain bounded for large $|z|$. In view of the theorem on removable singularities it follows that $u_{1}(z)$ and $u_{2}(z)$ are equal to holomorphic functions of $1 / z$ in a neighborhood of $1 / z=0$, which in turn implies that there are complex numbers $u_{1}(\infty), u_{2}(\infty)$ such that $u_{1}(z) \rightarrow u_{1}(\infty)$ and $u_{2}(z) \rightarrow u_{2}(\infty)$ as $|z| \rightarrow \infty$. In other words, $\Omega_{U}=\left\{\left(u_{1}(\infty), u_{2}(\infty)\right)\right\}$. Because the limit set $\Omega_{U}$ is invariant under the autonomous Hamiltonian system and contains only one point, this point is an equilibrium point of the autonomous Hamiltonian system. That is, $u_{2}(\infty)=0$ and $u_{1}(\infty)$ is equal to one of the two zeros $c$ of $u \mapsto 6 u^{2}+1$. According to the last statement in Corollary 4.6, $(-c, 0) \in \Omega_{U}$ if $(c, 0) \in \Omega_{U}$, where $(-c, 0) \neq(c, 0)$ because $c \neq 0$. This contradiction with $\Omega_{U}=\{(c, 0)\}$ completes the proof.

In Lemma 5.18 more information will be given about the asymptotic distribution for large $|\xi|$ of the poles $\xi$ of the solutions $y(x)$ of the first Painlevé equation. In the remainder of this section we discuss, for the solutions $U(z)$ close to the infinity set $I$, the asymptotic behavior of the set of $z$ 
such that $U(z)$ is close to $L_{i}^{(9-i)}$ for $0 \leq i \leq 3$, extending the description for $4 \leq i \leq 8$ in Lemma 4.2

As in Lemma 4.2, one finds concentric approximate discs $D_{1}, D_{2}$, and approximate discs $D_{0}$ of small radii such that the connected component of the set of all $z \in \mathbb{C}$ such that the solution in $S_{9}$ is close to $L_{1}^{(8)} \backslash L_{2}^{(7)}, L_{2}^{(7)} \backslash\left(L_{1}^{(8)} \cup L_{3}^{(6)}\right)$, and $L_{0}^{(9)} \backslash L_{3}^{(6)}$ is equal to $D_{1}, D_{2} \backslash D_{1}$, and $D_{0}$, respectively.

More precisely, $L_{1}^{(8)} \backslash L_{2}^{(7)}$ is visible in $\left(u_{121}, u_{122}\right)$ chart, where it is defined by $u_{121}=0$ and parametrized by $u_{122}$. We have $\dot{u}_{122} \sim u_{121}{ }^{-1}=w_{12}{ }^{-1 / 2} \sim q^{1 / 2}=d^{-1 / 2}$, where $\dot{q} / q=\dot{E} / E \sim$ $-6(5 z)^{-1}$, hence $d$ is approximately constant. Therefore each connected component of the set of all $z \in \mathbb{C}$ such that $\left|u_{122}(z)\right| \leq R_{1}$ is an approximate disc $D_{1}$ of radius $\sim|d|^{1 / 2} R_{1}$, and $z \mapsto u_{122}(z)$ is a complex analytic diffeomorphism from $D_{1}$ onto $\left\{u \in \mathbb{C}|| u \mid \leq R_{1}\right\}$. Furthermore, $L_{2}^{(7)} \backslash L_{3}^{(6)}$ is visible in $\left(u_{221}, u_{222}\right)$ chart, where it is defined by $u_{221}=0$ and parametrized by $u_{222}$, whereas the part of $L_{1}^{(8)}$ in this chart is defined by $u_{222}=0$ and parametrized by $u_{221}=u_{111}=u_{031} u_{032}{ }^{-1}=u_{122}{ }^{-1}$, hence $\left|u_{122}\right|>R_{1}$ corresponds to $\left|u_{221}\right|<R_{1}{ }^{-1}$. We have $\dot{u}_{222} \sim 2 u_{221}{ }^{-1}=2 w_{22}{ }^{-1 / 4} u_{222}{ }^{1 / 2}$, where $w_{22} \sim q^{-1}=d$ and $\dot{q} / q=\dot{E} / E \sim-6(5 z)^{-1}$ yields that $\left(u_{222}{ }^{1 / 2}\right)^{\bullet} \sim d^{-1 / 4}$ with approximately constant $d$, hence $u_{222}(z) \sim\left(u_{222}\left(z_{0}\right)^{1 / 2}+d^{-1 / 4}\left(z-z_{0}\right)\right)^{2}$. Let $R_{2}$ be a large finite positive real number. As $\left|u_{221}\right|<R_{1}^{-1}$ corresponds to $\left|u_{222}\right| \sim|d|^{1 / 2}\left|u_{221}\right|^{-2}>|d|^{1 / 2} R_{1}^{2}$, and $|d|^{1 / 4} /|d|^{1 / 2}>>$ 1, the mapping $z \mapsto u_{222}(z)$ is a twofold covering from the complement of $D_{1}$ in an approximate disc $D_{2}$ of radius $\sim|d|^{1 / 4} R_{2}{ }^{1 / 2}$ onto $\left\{\left.u \in \mathbb{C}|| d\right|^{1 / 2} R_{1}{ }^{2}<|u| \leq R_{2}\right\}$. Finally $L_{0}^{(9)} \backslash L_{3}^{(6)}$ is visible in the $\left(u_{021}, u_{022}\right)$ chart, where it is defined by $u_{021}=0$ and parametrized by $u_{022}$. We have $\dot{u}_{022} \sim 6 u_{021}{ }^{-1}=-6 w_{02}{ }^{-1 / 3}$, where $w_{02} \sim 4 q^{-1}=4 d$, and $\dot{q} / q=\dot{E} / E \sim-6(5 z)^{-1}$ yields that $d$ is approximately constant, with $\dot{u}_{022} \sim-2^{1 / 3} 3 d^{-1 / 3}$. Let $R_{0}$ be a large finite positive real constant. Then each connected component of the set of all $z \in \mathbb{C}$ such that $\left|u_{122}(z)\right| \leq R_{0}$ is an approximate disc $D_{0}$ of radius $\sim 2^{-1 / 3} 3^{-1}|d|^{1 / 3} R_{0}$, and $z \mapsto u_{122}(z)$ is a complex analytic diffeomorphism from $D_{0}$ onto $\left\{u \in \mathbb{C}|| u \mid \leq R_{0}\right\}$.

In order to understand the location of the concentric discs $D_{8} \subset D_{7} \subset D_{6} \subset D_{5} \subset D_{4}$, the concentric discs $D_{1} \subset D_{2}$ and the discs $D_{0}$ in the complex $z$-plane, we first describe the situation for the solutions of the autonomous Hamiltonian system, which have a similar behavior near the infinity set $I$, but in addition has the function $q=2 E$ as a constant of motion. Recall that for each $q \in \mathbb{C} \backslash\{ \pm \mathrm{i} \sqrt{8 / 27}\}$ the level set in $S_{9}(\infty)$ of the function $q$ is an elliptic curve $C_{q}$ such that $z \mapsto \Phi^{z}(\sigma(q))$ defines an isomorphism from $\mathbb{C} / P(q)$ onto $C_{q}$. Here $\Phi^{z}$ denotes the flow of the autonomous Hamiltonian system defined by the function $E=q / 2, \sigma(q)$ is the unique point in $C_{q} \cap L_{9}(\infty)$, and $P(q)$ is the period lattice of the flow at the level $q$. In the $\left(u_{1}, u_{2}\right)$ coordinate system, the first coordinate $u(z)=u_{1}\left(\Phi^{z}(\sigma(q))\right)$ of $\Phi^{z}(\sigma(q))$ is the solution of the autonomous differential equation $\mathrm{d}^{2} u / \mathrm{d} z^{2}=6 u^{2}+1$ such that $\dot{u}^{2}-4 u^{3}-2 u=q$ and $u(z)$ has a pole at $z \in P(q)$, and no other poles. Therefore $u(z)=\wp_{P(q)}(z)$, the Weierstrass $\wp$ function defined by the lattice $P(q)$.

Locally the period lattice has a $\mathbb{Z}$-basis $p_{1}(q), p_{2}(q)$ depending in a complex analytic fashion on $q$. The period functions $p(q)=p_{i}(q)$ satisfy the homogeneous linear second order differential equation

$$
\frac{\mathrm{d}^{2} p}{\mathrm{~d} q^{2}}+\frac{54 q}{8+27 q^{2}} \frac{\mathrm{d} p}{\mathrm{~d} q}+\frac{15}{4\left(8+27 q^{2}\right)} p=0
$$

as an application of Bruns [3, p. 237, 238] to $g_{2}(q)=-2$ and $g_{3}(q)=-q$.

Lemma 4.8. For large $|q|$ the period lattice $P(q)$ has a $\mathbb{Z}$-basis of the form

$$
\begin{gathered}
p_{1}(q)=q^{-1 / 6} a(1 / q)+q^{-5 / 6} b(1 / q), \\
p_{2}(q)=q^{-1 / 6} \mathrm{e}^{2 \pi \mathrm{i} / 6} a(1 / q)+q^{-5 / 6} \mathrm{e}^{-2 \pi \mathrm{i} / 6} b(1 / q), \\
15
\end{gathered}
$$


where $a$ and $b$ are complex analytic functions on an open neighborhood of the origin in the complex plane such that $a(0)=-\mathrm{i} 2^{-1} \pi^{-1} \Gamma(1 / 3)^{3}$ and $b(0)=\mathrm{i} 2^{4} 3^{-3 / 2} \pi^{2} \Gamma(1 / 3)^{-3}$. Here $\Gamma$ denotes Euler's Gamma function. The differential equation (4.15) in combination with the explicit values of $a(0)$ and $b(0)$ leads to a successive determination of the coefficients in the Taylor expansions at the origin of the functions $a$ and $b$.

For $0 \leq i \leq 8$ and $i \neq 3$ the point $\Phi^{z}(\sigma(q))$ is near $L_{i}^{(9-i)}$ if and only if $z$ belongs to the aforementioned approximate discs $D_{i}$ with $\delta=q^{-1}$. The centers of the concentric discs $D_{8} \subset D_{7} \subset$ $D_{6} \subset D_{5} \subset D_{4}$ are at the points of the period lattice $P(q)$ that are the pole points of the solution $u(z)=\wp_{P(q}(z)$ of the autonomous differential equation $\ddot{u}=6 u^{2}+1$. The centers of the concentric discs $D_{1} \subset D_{2}$ are at the zeros of $u(z)=\wp_{P(q)}(z)$. The zeros of $u(z)$ are close of order smaller than $|q|^{-1 / 6}$ to the points $\left(p_{1}(q)+p_{2}(q)\right) / 3$ and $2\left(p_{1}(q)+p_{2}(q)\right) / 3$ modulo $P(q)$. The centers of the discs $D_{0}$ are at the zeros of the derivative $\dot{u}(z)=\mathrm{d} \wp_{P(q)}(z) / \mathrm{d} z$, and close of order smaller than $q^{-1 / 6}$ to the points $p_{1}(q) / 2, p_{2}(q) / 2$, and $\left(p_{1}(q)+p_{2}(q)\right) / 2$ modulo $P(q)$.

Proof. Any period along a closed path $\gamma_{q}$ on the curve $\dot{u}^{2}=4 u^{3}+2 u+q$ is equal to

$$
p=\oint\left(4 u^{3}+2 u+q\right)^{-1 / 2} \mathrm{~d} u=4^{-1 / 3} q^{1 / 3} q^{-1 / 2} \int_{\gamma}\left(v^{3}+2 \cdot 4^{-1 / 3} q^{-2 / 3} v+1\right)^{-1 / 2} \mathrm{~d} v .
$$

Asymptotically for $q \rightarrow \infty$ we have

$$
\begin{aligned}
\left(v^{3}+2 \cdot 4^{-1 / 3} q^{-2 / 3} v+1\right)^{-1 / 2} & =\left(v^{3}+1\right)^{-1 / 2}\left(1+2 \cdot 4^{-1 / 3} q^{-2 / 3} v /\left(v^{3}+1\right)\right)^{-1 / 2} \\
& =\left(v^{3}+1\right)^{-1 / 2}-4^{-1 / 3} q^{-2 / 3}\left(v^{3}+1\right)^{-3 / 2} v+\mathrm{O}\left(q^{-4 / 3}\right) .
\end{aligned}
$$

Furthermore $\mathrm{d}\left(v^{3}+1\right)^{-1 / 2} / \mathrm{d} v=-\frac{1}{2}\left(v^{3}+1\right)^{-3 / 2} 3 v^{2}$, and therefore an integration by parts yields

$$
\oint\left(v^{3}+1\right)^{-3 / 2} v \mathrm{~d} v=-(2 / 3) \oint\left(\left(v^{3}+1\right)^{-1 / 2}\right)^{\prime} v^{-1} \mathrm{~d} v=-(2 / 3) \oint\left(v^{3}+1\right)^{-1 / 2} v^{-2} \mathrm{~d} v .
$$

Therefore $p(q)=q^{-1 / 6} \alpha+q^{-5 / 6} \beta+\mathrm{O}\left(q^{-3 / 2}\right)$, where

$$
\alpha=2^{-2 / 3} \oint\left(v^{3}+1\right)^{-1 / 2} \mathrm{~d} v \text { and } \beta=2^{-1 / 3} 3^{-1} \oint\left(v^{3}+1\right)^{-1 / 2} v^{-2} \mathrm{~d} v,
$$

where the integration is over a closed path on the elliptic curve $\dot{v}^{2}=v^{3}+1$ homotopic to $\gamma_{q}$. Here the elliptic curve is the compact one obtained by adding one point at infinity to the curve $\dot{v}^{2}=v^{3}+1$ in the affine $(v, \dot{v})$ plane. In the next computations we use the well-known formulas

$$
\begin{aligned}
\Gamma(p) & :=\int_{0}^{\infty} \mathrm{e}^{-t} t^{p-1} \mathrm{~d} t \\
\Gamma(p+1) & =p \Gamma(p) \\
\Gamma\left(\frac{1}{2}\right) & =\pi^{1 / 2} \\
\mathrm{~B}\left(p_{1}, p_{2}\right) & :=\int_{0}^{1} t^{p_{1}-1}(1-t)^{p_{2}-1} \mathrm{~d} t \\
& =\int_{0}^{\infty} s^{p_{1}-1}(s+1)^{-p_{1}-p_{2}} \mathrm{~d} s=\frac{\Gamma\left(p_{1}\right) \Gamma\left(p_{2}\right)}{\Gamma\left(p_{1}+p_{2}\right)} \\
\Gamma(2 p) & =2^{2 p-1} \pi^{-1 / 2} \Gamma(p) \Gamma\left(p+\frac{1}{2}\right), \quad \text { and } \\
\Gamma(p) \Gamma(1-p) & =\frac{\pi}{\sin (\pi p)}
\end{aligned}
$$


for Euler's Gamma function, where 4.20), 4.21), and 4.22 are Euler's Beta function, Legendre's duplication formula, and the reflection formula for the Gamma function, respectively. The second identity in 4.20 follows from the substitution of variables $t=s /(s+1)$

As our first loop we take the closed path which doubly covers the real $v$-interval from $-\infty$ to -1 . The substitution of variables $v=-(s+1)^{1 / 3}$ then yields in view of 4.20 and the other identities for the Gamma function that $\alpha= \pm \mathrm{i} 2^{1 / 3} 3^{-1} \mathrm{~B}(1 / 2,1 / 6)= \pm \mathrm{i} 2^{-1} \pi^{-1} \Gamma(1 / 3)^{3}$ and $\beta=\mp \mathrm{i} 2^{2 / 3} 3^{-2} \mathrm{~B}(1 / 2,5 / 6)=\mp \mathrm{i} 2^{4} 3^{-3 / 2} \pi^{2} \Gamma(1 / 3)^{-3}$.

As our second loop we take the closed path in $\dot{v}^{2}=v^{3}+1$ which doubly covers the real $v$-interval from -1 to 0 followed by the straight line in the $v$-plane from 0 to $\mathrm{e}^{-2 \pi \mathrm{i} / 6}$, where the substitution of variables $w=\mathrm{e}^{2 \pi \mathrm{i} / 3} v$ shows that the integral of $\left(w^{3}+1\right)^{-1 / 2} \mathrm{~d} w$ over the the second interval is equal to minus the integral of $\left(v^{3}+1\right)^{-1 / 2} \mathrm{~d} v$ over the first interval. The first and the second loop in $\dot{v}^{2}=v^{3}+1$ intersect each other once, at the point $(v, \dot{v})=(-1,0)$, where the intersection is transversal, and therefore the intersection number of the first loop with the second loop is equal to \pm 1 , where the sign depends on the choices of the orientations of the loops. as the elliptic curve is a real two-dimensional torus, its first homology group is isomorphic to $\mathbb{Z}^{2}$, when the fact that the intersection number of the two loops is \pm 1 implies that the homology classes of the two loops form a $\mathbb{Z}$-basis of the first homology group of the elliptic curve. This implies in turn that the corresponding periods, asymptotically equal to the integrals of $4^{-1 / 3} q^{-1 / 6} \dot{v}^{-1} \mathrm{~d} v$ over these loops, form a $\mathbb{Z}$-basis of the period lattice $P(q)$.

The substitution of variables $v=-s^{1 / 3}$ yields that the integral of $\left(v^{3}+1\right)^{-1 / 2} \mathrm{~d} v$ over the real $v$-interval from -1 to 0 is equal to $3^{-1} \int_{0}^{1}(1-s)^{-1 / 2} s^{-2 / 3} \mathrm{~d} s=3^{-1} \mathrm{~B}(1 / 2,1 / 3)$, and therefore the integral over the second loop leads to $\alpha=\left(1-\mathrm{e}^{2 \pi \mathrm{i} / 3}\right) 2^{1 / 3} 3^{-1} \mathrm{~B}(1 / 2,1 / 3)$. As

$$
\frac{\mathrm{B}(1 / 2,1 / 3)}{\mathrm{B}(1 / 2,1 / 6)}=\frac{\Gamma(1 / 2) \Gamma(1 / 3) \Gamma(2 / 3)}{\Gamma(1 / 2) \Gamma(1 / 6) \Gamma(5 / 6)}=\frac{\sin (\pi / 6)}{\sin (\pi / 3)}=3^{-1 / 2}
$$

and $\left(1-\mathrm{e}^{2 \pi \mathrm{i} / 3}\right) 3^{-1 / 2}=-\mathrm{i} \mathrm{e}^{2 \pi \mathrm{i} / 6}$, we arrive at the conclusion that, if in the above \pm we choose the minus sign, the second period is asymptotically equal to $\mathrm{e}^{2 \pi \mathrm{i} / 6}$ times the first period.

Because the differential equation (4.15) for the periods has $q=\infty$ as a regular singular point, and the two solutions $\lambda=-1 / 6$ and $\lambda=-5 / 6$ of its indicial equation $\lambda(\lambda-1)+2 \lambda+5 / 36=0$ do not differ by an integer, each solution of 4.15 is of the form $q^{-1 / 6} a(1 / q)+q^{-5 / 6} b(1 / q)$, where $a$ and $b$ are complex analytic functions on a neighborhood of the origin, and the solution is uniquely determined by $a(0)$ and $b(0)$. See for instance Coddington and Levinson [4, Ch. 4, Sec. 4]. It follows that our first period fits the description for $p_{1}(q)$. The analytic continuation of $p_{1}(q)$, when the small $1 / q$ runs around the origin once, is equal to $p_{2}(q)$. Because $p_{2}(q)$ asymptotically agrees with our second period, it is equal to it. Therefore the periods $p_{1}(q)$ and $p_{2}(q)$ described in the lemma form a $\mathbb{Z}$-basis of the period lattice $P(q)$. This completes the proof of the first paragraph in the lemma.

For the second paragraph we observe that we took as the center of the discs $D_{8}$ the pole points. The line $L_{1}^{(8)} \backslash L_{2}^{(7)}$ is visible in the $\left(u_{121}, u_{122}\right)$ chart, where it is defined by $u_{121}=0$ and is parametrized by $u_{122}$. As $u_{121}=u_{2}{ }^{-1}$ and $u_{122}=u_{1}$, it follows that the centers of the discs $D_{1}$, which correspond to $u_{122}=0$, correspond to the zeros of $u(z)=\wp_{P(q)}(z)$. The line $L_{0}^{(9)} \backslash L_{3}^{(6)}$ is visible in the $\left(u_{021}, u_{022}\right)$ chart, where it is defined by $u_{021}=0$ and parametrized by $u_{022}$. As $u_{021}=u_{1}{ }^{-1}$ and $u_{022}=u_{1}{ }^{-1} u_{2}$, it follows that the centers of the discs $D_{0}$ correspond to the zeros of the derivative $u_{2}(z)=\dot{u}(z)=\mathrm{d} \wp_{P(q)}(z) / \mathrm{d} z$ of the solution $u(z)=\wp_{P(q)}(z)$ of the autonomous differential equation $\ddot{u}=6 u^{2}+1$.

For large $|q|$ and $z$ not in one of the aforementioned small discs $D_{i}$, the solution of the autonomous Hamiltonian system in $S_{9} \backslash I$ is close to the part $L_{3}^{(6)}$ of $I$. In the $\left(u_{311}, u_{312}\right)$ chart, $L_{3}^{(6)}$ is defined by $u_{312}=0$ and parametrized by $u_{311}$. The base point $\left(u_{311}, u_{312}\right)=(4,0)$ corresponds to 
$L_{3}^{(6)} \cap L_{4}^{(5)}$, the origin $\left(u_{311}, u_{312}\right)=(0,0)$ to $L_{3}^{(6)} \cap L_{0}^{(6)}$, whereas $\left(u_{311}, u_{312}\right)=(\infty, 0)$ corresponds to $\left(u_{321}, u_{322}\right)=(0,0)$ hence to $L_{3}^{(6)} \cap L_{2}^{(7)}$. We have $u_{311}(z)=u(z)^{-3} \dot{u}(z)^{2}$ and $\dot{u}^{2}-4 u^{3}-2 u=q=$ constant, which suggests the rescaling $z=z_{0}+q^{-1 / 6} t$ and $u(z)=q^{1 / 3} v\left(q^{1 / 6}\left(z-z_{0}\right)\right)$. Then $u_{311}=v^{-3}\left(v^{\prime}\right)^{2}, v^{\prime \prime}=6 v^{2}+q^{-2 / 3}$, and $\left(v^{\prime}\right)^{2}=4 v^{3}+1+2 q^{-2 / 3} v$, hence $u_{311}=4+v^{-3}+2 q^{-2 / 3} v^{-2}$. In the limit $q=\infty$ this leads to $u_{311}(t)=v(t)^{-3} v^{\prime}(t)^{2}=4+v(t)^{-3}$. The equation $\left(v^{\prime}\right)^{2}=4 v^{3}+1$ has a regular hexagonal period lattice $P$, and we arrange that $v(t)=\wp(t)$ is the solution with its poles at the points of $P$. As the poles have order two, it follows that the mapping $\mathbb{C} / P \rightarrow L_{3}^{(6)}: t+P \mapsto$ $u_{311}(t)$ is a sixfold branched covering, where near the point $t+P=0+P$ the mapping behaves as $u_{311}(t)-4 \sim t^{6}$. At this ramification point $t+P$ all the six branches come together, where the image point $u_{311}=4$ corresponds to the centers of the discs $D_{8}$. There are three ramification points $t$ where $v \in \mathbb{C} \backslash\{0\}, v^{\prime}=0$, at each of which $u_{311}^{\prime}=0$ and $u_{311}^{\prime \prime}=2 v^{-3} v^{\prime \prime}=12 v^{-1} \neq 0$, which means that at each of these ramification points two branches come together. Both ramification points ly over $u_{311}=0$, corresponding to the centers of the discs $D_{0}$. The only other ramification points $t$ occur when $v=0$. There are two of these and at each one three branches come together. Both these ramification points ly over $u_{311}=\infty$, corresponding to the centers of the discs $D_{1}$.

The group of deck transformations of the aforementioned branched covering is isomorphic to $\mathbb{Z} / 6 \mathbb{Z}$ and generated by $T: z+P \mapsto \mathrm{e}^{2 \pi \mathrm{i} / 6} z+P$, which is an automorphism of $\mathbb{C} / P$ because $P$ has a $\mathbb{Z}$-basis consisting of $p_{1} \in \mathbb{C} \backslash\{0\}$ and $p_{2}=\mathrm{e}^{2 \pi \mathrm{i} / 6} p_{1}$. The ramification points of order 6 are the fixed points in $\mathbb{C} / P$ of $T$, which is the single point $0+P$. The ramification points of order 3 are the fixed points of $T^{2}$ which are no fixed points of $T$, which are the two points $\left(p_{1}+p_{2}\right) / 3+P$ and $2\left(p_{1}+p_{2}\right) / 3+P$. The ramification points of order 2 are the fixed points of $T^{3}: t+P \mapsto-t+P$ which are no fixed points of $T$, which are the three points $p_{1} / 2+P, p_{2} / 2+P$, and $\left(p_{1}+p_{2}\right) / 2+P$.

Figure 4.1 exhibits the complex times $z$ for which the solutions of the autonomous Hamiltonian system for large $q$ are near the various irreducible components of the singular fiber $I(\infty)$ over $q=\infty$. The solid dots represent the concentric discs $D_{i}, 4 \leq i \leq 8$, centered at the points of the period lattice $P(q)$ where $u_{1}$ has a pole, with respective radii $\sim|q|^{-1 /(9-i)} r_{i}$ for large finite $r_{i}$, where the distance between the points of $P(q)$ the period lattice is of order $|q|^{-1 / 6} \gg|q|^{-1 / 5} \gg \ldots \gg|q|^{-1}$. The solution is near $L_{8}^{(1)}$ when $z \in D_{8}$, and near $L_{i}^{(9-i)} \backslash L_{i+1}^{(10-i)}$ when $z \in D_{i} \backslash D_{i+1}$ for $4 \leq i \leq 7$. The double circles represent the concentric disks $D_{1}$ and $D_{2}$, centered at the zeros of $u_{1}$, approximately equal to the points $\left(p_{1}(q)+p_{2}(q)\right) / 3$ and $2\left(p_{1}(q)+p_{2}(q)\right) / 3$ modulo $P(q)$, and of radius $\sim|q|^{-1 / 2} r_{1}$ and $\sim|q|^{-1 / 4} r_{2}$ for large finite $r_{i}$, respectively. The solution is near $L_{1}^{(8)}$ when $z \in D_{1}$, and near $L_{2}^{(7)} \backslash L_{1}^{(8)}$ when $z \in D_{2} \backslash D_{1}$. The single circles represent the concentric discs $D_{0}$ centered at the zeros of $u_{2}=\dot{u}_{1}$, approximately equal to the points $p_{1}(q) / 2, p_{2}(q) / 2,\left(p_{1}(q)+p_{2}(q)\right) / 2$ modulo $P(q)$ with radius $\sim|q|^{-1 / 3} r$ for a large finite $r$. The solution is near $L_{0}^{(9)}$ when $z \in D_{0}$. When $z$ is in the complement of all the aforementioned discs, which happens for most of the complex times, the solution is near the component $L_{3}^{(6)}$ of $I(\infty)$.

Figure 4.2 is a contour plot of the absolute value of the Weierstrass $\wp$ function defined by the regular hexagonal lattice generated by 1 and $\frac{1}{2}+\frac{1}{2} \sqrt{3}$ i. The areas close to the lattice points $=$ the pole points of $\wp(z)$ should have been blacker than black, because the second order poles are very big. Instead the computer program has left these areas blank. The solid level curve is the one of the level at the zeros of $\wp^{\prime}(z)$, the saddle points of $|\wp(z)|$. For our $u(z)=\wp_{P(q)}(z)$ the lattice $P(z)$ is asymptotically equal to $q^{-1 / 6}$ times a standard regular hexagonal lattice $P_{0}$, and therefore $u(z) \sim q^{1 / 3} \wp_{P_{0}}(z)$. It follows that the pits in the mountain landscape of $|u(z)|$ in which the zeros of $u(z)$ ly are separated from each other by mountain ridges in which the heights of the passes, situated at the zeros of $\mathrm{d} u(z) / \mathrm{d} z$, are of large order $|q|^{1 / 3}$. 
The following lemma implies that the solutions near the part $L_{3}^{(6)} \backslash\left(L_{0}^{(9)} \cup L_{2}^{(7)} \cup L_{4}^{(5)}\right)$ of $I$ of the non-autonomous Boutroux-Painlevé system closely follow the solutions of the autonomous Hamiltonian system.

Lemma 4.9. Let $K$ be a compact subset of $L_{3}^{(6)} \backslash\left(L_{0}^{(9)} \cup L_{2}^{(7)} \cup L_{4}^{(5)}\right)$ and $R \in \mathbb{R}_{>0}$. Then there exists a neighborhood $U$ of $K$ in $S_{9}$ and a constant $C$ such that the distance between $x(z)$ and $x_{0}(z)$ is $\leq C\left|z_{0}\right|^{-1}|q|^{-1 / 6}$ if $\left|z_{0}\right| \geq C,\left|z-z_{0}\right| \leq R, x_{0}$ is a solution of the autonomous Hamiltonian system such that $x_{0}\left(z_{0}\right) \in U$ and $x_{0}(z) \in U$, and $x$ is the solution of the Boutroux-Painlevé system such that $x\left(z_{0}\right)=x_{0}\left(z_{0}\right)$.

Proof. $L_{3}^{(6)} \backslash\left(L_{0}^{(9)} \cup L_{2}^{(7)} \cup L_{4}^{(5)}\right)$ is visible in the $\left(u_{311}, u_{312}\right)$ chart, where it is determined by $u_{312}=0$ and parametrized by $u_{311}$. As $u_{311}=\infty, u_{311}=0$, and $u_{311}=4$ correspond to $L_{2}^{(9)}, L_{0}^{(9)}$, and $L_{4}^{(5)}$, respectively, we keep $u_{311}$ bounded and bounded away from 0 and 4 . We multiply the vector field by the scalar factor $q^{-1 / 6}=u_{311}{ }^{1 / 2} u_{312}\left(u_{311}-4-2 u_{311^{2}} u_{312}\right)^{-1 / 6}$, which amounts to a time reparametrization along each solution curve. Lemma 4.8 implies that the distance between the periods of the solutions of the autonomous system is of order $|q|^{-1 / 6}$. Lemma 4.1 implies that, along the solutions near $L_{3}^{(6)}$, we have $q(z)=q\left(z_{0}\right)\left(z / z_{0}\right)^{-6 / 5+\mathrm{o}(1)}$, which is asymptotically constant for $\left|z-z_{0}\right|<<\left|z_{0}\right|$. This leads to the time rescaled system

$$
\begin{aligned}
\mathrm{d} u_{311} / \mathrm{d} t= & u_{311}{ }^{1 / 2}\left(12-3 u_{311}+2 u_{311}{ }^{2} u_{312}{ }^{4}\right)\left(u_{311}-4-2 u_{311}{ }^{2} u_{312}{ }^{4}\right)^{-1 / 6} \\
\mathrm{~d} u_{312} / \mathrm{d} t= & -3 u_{311}{ }^{1 / 2}\left(u_{311}-4\right)^{5 / 6}, \\
& +(5 z)^{-1 / 2} u_{311}{ }^{1 / 2} u_{312}{ }^{2}\left(u_{311}-4-2 u_{311}{ }^{2} u_{312}{ }^{4}\right)^{-1 / 6} .
\end{aligned}
$$

Actually, we have six vector fields, one for each of the sixth roots of $1 / q$, corresponding to the fact that the curves $q$ equal to a large constant pass six times near $L_{3}^{(6)} \backslash\left(L_{0}^{(9)} \cup L_{2}^{(7)} \cup L_{4}^{(5)}\right)$. The autonomous Hamiltonian system is obtained from 4.23 by deleting the $z$-dependent term from the equation for $\mathrm{d} u_{312} / \mathrm{d} t$. As $u_{312} \sim q^{-1 / 6} u_{311}{ }^{-1 / 2}\left(u_{311}-4\right)^{1 / 6}$, the difference between the two vector fields is $\sim(5 z)^{-1} q^{-1 / 3} u_{311}^{-1 / 2}\left(u_{311}-4\right)^{1 / 6}=\mathrm{O}\left(z^{-1} q^{-1 / 3}\right)$, and therefore $\left|x(z)-x_{0}(z)\right|=$ $\mathrm{O}\left(q^{-1 / 3} z^{-1}\left(t-t_{0}\right)\right)$, where $t-t_{0}=\mathrm{O}\left(q^{1 / 6}\left(z-z_{0}\right)\right)$.

It follows from the first equation in 4.23 that, for the solutions near $L_{3}^{(6)} \backslash\left(L_{0}^{(9)} \cup L_{2}^{(7)} \cup L_{4}^{(5)}\right)$ of both the non-autonomous and the autonomous system, the $t$-times needed to go from a position with first coordinate $u_{311}$ to a position near $L_{3}^{(6)} \cap L_{0}^{(9)}, L_{3}^{(6)} \cap L_{2}^{(7)}$, and $L_{3}^{(6)} \cap L_{4}^{(5)}$ are asymptotically equal to the integral of $-3^{-1} U^{-1 / 2}(U-4)^{-5 / 6}$ over the $U$-interval from $u_{311}$ to $0, \infty$, and 4 , respectively. Therefore the distances between the discs $D_{i}$ for $x$ and the discs $D_{i}$ for $x_{0}$ are of smaller order than $|q|^{1 / 6}$. As the distances between the discs for $x_{0}$ are of order $|q|^{1 / 6}$, where near $L_{3}^{(6)}$ we have $q=d$, see Lemma 4.2, the discs for $x$ have asymptotically the same relative position as the discs for $x_{0}$. Therefore Lemma 4.9 leads to a description of the solutions of the Boutroux-Painlevé system near $I$ which closely resembles the description of the the solutions of the autonomous Hamiltonian system near $S_{9, \infty}$. Lemma 4.1 implies for the solutions of the Boutroux-Painlevé system near $L_{3}^{(6)}$ we have $q(z) \sim q\left(z_{0}\right)\left(z / z_{0}\right)^{-6 / 5+\mathrm{o}(1)}$ hence the order $|q(z)|^{-1 / 6} \sim\left|q\left(z_{0}\right)\right|^{-1 / 6}\left(|z| /\left|z_{0}\right|\right)^{1 / 5+\mathrm{o}(1)}$ of the distances between the discs increases slowly with growing $|z| \geq\left|z_{0}\right|>>1$, until the solution has left the neighborhood of $I$ in $S_{9}$ where the estimates hold.

Remark 4.10. Boutroux [2, bottom of p. 310] claimed that the quantity $\left(Y^{\prime}\right)^{2}-4 Y^{3}+12 Y$, see Remark 2.1, remains bounded when $X$ runs to infinity along any path with bounded argument not passing through any pole point of $Y(X)$. As the function $q$ has a simple pole at every pole point of $u(z)$, where $u_{1}(z) u_{2}(z)$ and $u_{1}(z)^{2}$ have poles of order 5 and 4 , respectively, it follows from (2.5) that $\left(Y^{\prime}\right)^{2}-4 Y^{3}+12 Y$ has a pole of order 5 at every pole point of $Y(X)$, and therefore the 
claim of Boutroux can only be valid if the final $X$ stays sufficiently far away from the pole points of $Y(X)$. Boutroux referred for the proof to [2, §10], which in turn refers back to the estimates on [2. p. 296, 297]. No proof is given for the existence of paths along which estimates of the form [2, (17) on p. 296] hold, and of which the concatenation is the desired path in the $X$-plane running to infinity. In particular no analysis is given of the existence of paths along which estimates of the form [2, (17) on p. 296] hold when $|D|$ is large. Boutroux did not give an explicit 6/5 power law as in Corollary 4.3, although the last term in the right hand side of [2, (36) on p. 321] contains a factor $X^{-6 / 5}$ which Boutroux used in order to argue that the quantity $\left(Y^{\prime}\right)^{2}-4 Y^{3}+12 Y$ remains bounded.

The asymptotic formula of Joshi and Kruskal [16, (5.18) with $c=6 / 5$ ] implies that the solution $\mathcal{E}$ of the averaged equation for their quantity $E$, which corresponds to our energy function $E$, satisfies $\mathcal{E}(z) \sim \mathcal{E}\left(z_{0}\right)\left(z / z_{0}\right)^{-6 / 5+\mathrm{o}(1)}$ for large $|\mathcal{E}|$. Joshi and Kruskal [16] did not provide estimates for $E$ in terms of $\mathcal{E}$. It follows from (3.3) that $E$ has a pole of order one at the pole points $z=\zeta$, and Lemma 4.9 together with the paragraph preceding it imply that for the solutions near the infinity set the pole points form an approximate regular hexagonal lattice with distance between the pole points of order $|E|^{-1 / 6}$. Therefore we cannot have $E(z) \sim E\left(z_{0}\right)\left(z / z_{0}\right)^{-6 / 5+\mathrm{o}(1)}$ for all large $|E|$. However, Lemma 4.2 and Corollary 4.3 imply this estimate if $z$ stays away from the pole points at a distance of larger order than $|d|$, when $|d| \sim|2 E|^{-1}<<|E|^{-1 / 6}$.

\section{NEAR THE EQUILIBRIA OF THE LIMIT SYSTEM}

In this section, we consider the Boutroux-Painlevé system near the equilibria of its autonomous limit and prove several results about the solutions near these equilibria. These are the solutions called tronquée by Boutroux that are asymptotically free of poles near infinity in certain sectors. We use classical methods to determine these properties and end with a determination of their sequences of poles near the boundaries of pole-free sectors.

The limit system

$$
\begin{aligned}
& \dot{u}_{1}=u_{2}, \\
& \dot{u}_{2}=6 u_{1}^{2}+1,
\end{aligned}
$$

obtained from 2.2 by substituting $z=\infty$, has two equilibrium points, determined by $u_{1}=\epsilon \mathrm{i} / \sqrt{6}$, $u_{2}=0$, where $\epsilon \in\{-1,1\}$. The linearization of the vector field at these equilibrium points is given by $\left(\delta u_{1}, \delta u_{2}\right) \mapsto\left(\delta u_{2}, 12 u_{1} \delta u_{1}\right)=\left(\delta u_{2}, \epsilon 2 \sqrt{6}\right.$ i $\left.\delta u_{1}\right)$, which has the eigenvalues

$$
\lambda_{ \pm}= \pm(24)^{1 / 4} \mathrm{e}^{\pi \mathrm{i}(1 / 2-\epsilon / 4)}
$$

with the corresponding eigenspaces determined by $\delta u_{2}=\lambda_{ \pm} \delta u_{1}$.

Because the vector field in $S_{9}(\infty) \backslash I(\infty)$ has no zeros on the pole line $L_{9}(\infty)$, and $S_{9}(\infty) \backslash$ $\left(I(\infty) \cup L_{9}(\infty)\right)$ is equal to the coordinate neighborhood where $u_{1}$ and $u_{2}$ are finite, these are the only equilibrium points of the autonomous Hamiltonian system in $S_{9}(\infty) \backslash I(\infty)$. The values of the function $q:=2 E$ in $(2.3)$ at the equilibrium points are equal to $-\epsilon(2 / 3)^{3 / 2} \mathrm{i}$, and the curves $q=-\epsilon(2 / 3)^{3 / 2} \mathrm{i}$ are the only singular level curves of the function $q$ in $S_{9}(\infty) \backslash I(\infty)$. Both these singular fibers of the elliptic fibration $q: S_{9}(\infty) \rightarrow \mathbb{P}^{1}$ are of Kodaira type $\mathrm{I}_{1}$. The fiber $q=\infty$, equal to the infinity set $I(\infty)$ of the Hamiltonian vector field defined by the Hamiltonian function $E=q / 2$, is of Kodaira type $\mathrm{II}^{*}$. The configuration of the singular fibers of the elliptic fibration $q: S_{9}(\infty) \rightarrow \mathbb{P}^{1}$ is $\mathrm{II}^{*}+\mathrm{I}_{1}+\mathrm{I}_{1}$.

5.1. Perturbation of a system with a hyperbolic equilibrium point. With the substitution $t=\lambda_{+} z$ and an affine change of coordinates $\left(u_{1}, u_{2}\right) \mapsto\left(p^{+}, p^{-}\right)$which maps $(\epsilon \mathrm{i} / \sqrt{6}, 0)$ to $(0,0)$ and the eigenvectors of the linearization of $(5.1)$ at $(\epsilon \mathrm{i} / \sqrt{6}, 0)$ for the eigenvalues $\lambda_{+}$and $\lambda_{-}$to $(1,0)$ and $(0,1)$, respectively, the system 2.2$)$ is transformed to a system

$$
\mathrm{d} p / \mathrm{d} t=v\left(t^{-1}, p\right)
$$


such that the right hand side satisfies the conditions in Lemma 5.1 below. In the remainder of this section we discuss arbitrary systems (5.3) which satisfy the conditions in Lemma 5.1. In the lemmas 5.2 - 5.11, we describe the solutions $p(t)$ of 5.3 which remain bounded for all $t$ in an unbounded domain in the complex plane, where the domain is increased step by step.

The conclusions of the lemmas 5.2 - 5.11 follow from O. and R. Costin [7, Th. 1 and 2], which deals with systems (5.3) in arbitrary dimensions $n$, where $v$ is complex analytic on an open neighborhood of $(0,0)$ in $\mathbb{C} \times \mathbb{C}^{n}, v(0,0)=0$, and less special assumptions are made on $L_{0}:=\partial v(0, p) /\left.\partial p\right|_{p=0}$. The proof of [7, Th. 1] uses Borel summation. Our proofs, a sequence of variations on the method of Cotton, are more classical, where our step by step approach may be helpful in understanding all the aspects of the final picture. As we only need the case that $n=2$ and $L_{0}$ has the eigenvalues \pm 1 , and a wide generalization would require more elaborate notations and proofs, we did not attempt to write down the latter. In the next paragraph we summarize the results of the lemmas $5.2-5.11$.

In Lemma 5.2 we prove that the solutions $p(t)$ of $(5.3)$ which remain small on half lines $t_{0}+\mathbb{R}_{\geq 0}$ form a one-parameter family parametrized by the complex number $p\left(t_{0}\right)^{-}$. Lemma 5.3 yields unique solutions $p_{\uparrow}(t)$ and $p_{\downarrow}(t)$ which remain small on horizontal axes in the upper and lower complex half plane, respectively. In Lemma 5.5 it is established that for every $\epsilon>0$ there exists an $r>0$ such that the $p_{\uparrow}(t)$ have a common complex analytic extension to the domain defined by the inequalities $|t|>r$ and $-\pi / 2+\epsilon<\arg t<3 \pi / 2-\epsilon$, and that this common extension has an asymptotic expansion in strictly negative powers of $t$ as $|t| \rightarrow \infty$. Lemma 5.6 states that there exists an $\alpha \in \mathbb{C}$ such that for each solution $p(t)$ in Lemma 5.2 there exist $\eta, r>0$ such that $p(t)$ has a complex analytic extension to a small solution of (5.3) on the domain $R_{\eta, r}$ defined by $|t|>r,-\pi<\arg t<\pi$, and $|\tau(t)|<\eta$, where $\tau(t):=\mathrm{e}^{-t} t^{\alpha}$. Furthermore, there exist unique $C \in \mathbb{C}$ and $d_{j} \in \mathbb{C}^{2}, j \in \mathbb{Z}_{\geq 0}$, such that on every subdomain $\Sigma$ where $\mathrm{e}^{-t}$ is of smaller order than every negative power of $t$ as $|t| \rightarrow \infty$ the function $p(t)-p_{\uparrow}(t)$ has the asymptotic expansion $p(t)-p_{\uparrow}(t) \sim C \mathrm{e}^{-t} t^{\alpha} \sum_{j=0}^{\infty} t^{-j} d_{j}$. Here the $d_{j}$ do not depend on the choice of the solution $p(t)$ in Lemma 5.2. With a similar argument as in the proof of Lemma 5.6, we obtain that in a subdomain where $C_{1}|t|^{-\epsilon_{1}}<\left|\mathrm{e}^{-t}\right|<C_{2}|t|^{-\epsilon_{2}}$ for strictly positive $C_{1}, C_{2}, \epsilon_{1}, \epsilon_{2}$, we have an asymptotic expansion of the form $p(t) \sim \sum_{h, i \in \mathbb{Z}_{\geq 0}} \tau(t)^{h} t^{-i} p_{h, i}$ as $|t| \rightarrow \infty$. Here the coefficients $p_{h, i}$ satisfy $p_{h, i}=C^{h} c_{h, i}$, where the coefficients $c_{h, i}$ do not depend on the choice of the solution $p(t)$ in Lemma 5.3. Lemma 5.8 states that for each $i$ the series $F_{i}(\tau):=\sum_{h \geq 0} \tau^{h} p_{h, i}$ converges for sufficiently small $|\tau|$, when Lemma 5.11 implies that we have an asymptotic expansion of the form $p(t) \sim \sum_{i \geq 0} F_{i}(\tau(t)) t^{-i}$ as $|t| \rightarrow \infty$ in a domain where $\operatorname{Im} t \geq 0$ and $F_{0}(\tau(t))$ remains bounded, extending far into the domain where $p(t)$ is bounded away from zero. This expansion will lead to the asymptotic determination of [7, Proposition 15] of the sequence of poles of the truncated solutions of (1.1) in Lemma 5.18. At some places our statements are more precise and our proofs more complete than those in [7].

Lemma 5.1. Assume that $v=\left(v^{+}, v^{-}\right)$is a $\mathbb{C}^{2}$-valued complex analytic function on an open neighborhood $D$ of the origin in $\mathbb{C}^{3}$ such that $v^{ \pm}(u, p)= \pm p^{ \pm}+w(u, p), w(0,0)=0$, and $\partial w(0, p) /\left.\partial p\right|_{p=0}=0$. Here $p=\left(p^{+}, p^{-}\right) \in \mathbb{C}^{2}$ and $\|p\|:=\max \left\{\left|p^{+}\right|,\left|p^{-}\right|\right\}$. Then there exist strictly positive real numbers $\delta_{0}, \epsilon_{0}, C_{1}, C_{2}, C_{3}$, and $C_{4}$ such that $\|w(u, p)\| \leq C_{1}\|p\|^{2}+C_{2}|u|$, and $\|\partial w(u, p) / \partial p\| \leq C_{3}\|p\|+C_{4}|u|$ if $\|p\|<\delta_{0}$ and $|u|<\epsilon_{0}$. Here the last condition implies the preceding one for $C_{1}=C_{3} / 2$ and a suitable $C_{2}$. In the sequel we will take $D$ equal to the set of all $(u, p) \in \mathbb{C} \times \mathbb{C}^{2}$ such that $|u|<\epsilon_{0}$ and $\|p\|<\delta_{0}$. For solutions $p$ of (5.3) we will always require that $|t|>1 / \epsilon_{0}$ and $\|p(t)\|<\delta_{0}$ for all $t$ in the domain of definition of $p$.

Proof. We have $\|w(u, p)\| \leq\|w(u, p)-w(0, p)\|+\|w(0, p)\|$ and, with the notation $\partial_{2} w(u, p)=$ $\partial w(u, p) / \partial p,\left\|\partial_{2} w(u, p)\right\| \leq\left\|\partial_{2} w(u, p)-\partial_{2} w(0, p)\right\|+\left\|\partial_{2} w(0, p)\right\|$. Because $w(0,0)=0$ and 
$\partial_{2} w(0,0)=0$, an application of a Taylor expansion with estimate for the remainder term to each of the terms between the norm signs yields the estimates in Lemma 5.1 .

Lemma 5.2. In the situation of Lemma 5.1, let $0<\delta<\delta_{0}, 0<\epsilon<\epsilon_{0}, t_{0}, a^{-} \in \mathbb{C},|t| \geq 1 / \epsilon$ for every $t \in T:=t_{0}+\mathbb{R}_{\geq 0},\left|a^{-}\right|+C_{1} \delta^{2}+C_{2} \epsilon<\delta$, and $C_{3} \delta+C_{4} \epsilon<1$. Then there exists a unique $a^{+}=a_{t_{0}, a^{-}}^{+} \in \mathbb{C}$ such that the solution $p(t)$ of the differential equation $\sqrt{5.3)}$ with $p\left(t_{0}\right)=\left(a^{+}, a^{-}\right)$is defined for all $t \in T$ and satisfies $\|p(t)\| \leq \delta$ for every $t \in T$. For any $0<\delta<\min \left\{\delta_{0}, 1 / C_{1}, 1 / C_{3}\right\}$, the set of all $\left(t_{0}, a^{-}\right)$for which the above conclusions hold is a non-empty open subset of $\mathbb{C}^{2}$ on which the function $\left(t_{0}, a^{-}\right) \mapsto a_{t_{0}, a^{-}}^{+}$is complex analytic. Similar conclusions hold with $t_{0}+\mathbb{R}_{\geq 0}$ replaced by a curve $\tau+\mathrm{i} \sigma(\tau)$, where $\tau \in\left[\operatorname{Re} t_{0}, \infty\left[, \sigma:\left[\operatorname{Re} t_{0}, \infty[\rightarrow \mathbb{R}\right.\right.\right.$ is continuously differentiable with a bounded derivative, and the curve stays sufficiently far away from the origin.

Proof. We apply the method of Cotton [8].

The system (5.3) can be viewed as an inhomogeneous system of linear differential equations with $w\left(t^{-1}, p(t)\right)$ as the inhomogeneous term, and therefore it is equivalent to the system of integral equations

$$
p(t)^{ \pm}=\mathrm{e}^{ \pm(t-\tau)} p(\tau)^{ \pm}+\int_{\tau}^{t} \mathrm{e}^{ \pm(t-s)} w\left(s^{-1}, p(s)\right)^{ \pm} \mathrm{d} s .
$$

Let $\mathcal{X}$ denote the set of all continuous functions $p: T \rightarrow \mathbb{C}^{2}$ such that $\left(t^{-1}, p(t)\right) \in D$ for every $t \in T$ and $t \mapsto w\left(t^{-1}, p(t)\right)^{+}$is bounded on $T$. If the solution $p(t)$ of (5.3) belongs to $\mathcal{X}$, then we can let $\tau \in T$ run to infinity in the equation (5.4) for $\pm=+$, and obtain

$$
p(t)^{+}=-\int_{t}^{+\infty} \mathrm{e}^{t-s} w\left(s^{-1}, p(s)\right)^{+} \mathrm{d} s,
$$

where $\int_{t}^{+\infty}$ indicates the limit for $T \ni \tau \rightarrow \infty$ of $\int_{t}^{\tau}$. Conversely, if $a^{-} \in \mathbb{C}$ and $p \in \mathcal{X}$, then the equations (5.5) and

$$
p(t)^{-}=\mathrm{e}^{t_{0}-t} a^{-}+\int_{t_{0}}^{t} \mathrm{e}^{s-t} w\left(s^{-1}, p(s)\right)^{-} \mathrm{d} s
$$

for all $t \in T$ imply that $p(t)$ is a solution of 5.3 such that $p\left(t_{0}\right)^{-}=a^{-}$.

Let $F$ denote the integral operator which assigns to each $p \in \mathcal{X}$ the function $F(p): T \rightarrow \mathbb{C}^{2}$ such that, for each $t \in T, F(p)(t)^{+}$and $F(p)(t)^{-}$are equal to the right hand side of (5.5) and (5.6), respectively. Let $X$ denote the set of all continuous functions $p: T \rightarrow \mathbb{C}^{2}$ such that $\|p(t)\| \leq \delta$ for every $t \in T$. Then $X \subset \mathcal{X}, X$ is a complete space with respect to the metric $d\left(p_{1}, p_{2}\right):=$ $\sup _{t \in T}\left\|p_{1}(t)-p_{2}(t)\right\|$, and the assumed estimates imply that

$$
\begin{aligned}
\left|F(p)(t)^{+}\right| & \leq \int_{0}^{\infty} \mathrm{e}^{-\tau}\left(C_{1} \delta^{2}+C_{2} \epsilon\right) \mathrm{d} \tau \leq \delta, \\
\left|F(p)(t)^{-}\right| & \leq \mathrm{e}^{t_{0}-t}\left|a^{-}\right|+\int_{0}^{\infty} \mathrm{e}^{-\tau}\left(C_{1} \delta^{2}+C_{2} \epsilon\right) \mathrm{d} \tau \leq \delta, \\
\left|F\left(p_{1}\right)(t)^{ \pm}-F\left(p_{2}\right)(t)^{ \pm}\right| & \leq \int_{0}^{\infty} \mathrm{e}^{-\tau}\left(C_{3} \delta+C_{4} \epsilon\right)\left\|p_{1}\left(t_{0}+\tau\right)-p_{2}\left(t_{0}+\tau\right)\right\| \mathrm{d} \tau \\
& \leq\left(C_{3} \delta+C_{4} \epsilon\right) d\left(p_{1}, p_{2}\right)
\end{aligned}
$$

for every $p, p_{1}, p_{2} \in X$ and $t \in T$. Therefore $F(X) \subset X$ and $F: X \rightarrow X$ is a contraction, with contraction factor $\leq C_{3} \delta+C_{4} \epsilon<1$, when the contraction mapping theorem implies that $F$ has a unique fixed point, a unique $p \in X$ such that $F(p)=p$. It follows that there is a unique solution $p(t)=p_{t_{0}, a^{-}}(t)$ of $(5.3)$ on $T$ such that $p\left(t_{0}\right)^{-}=a^{-}$and $\|p(t)\| \leq \delta$ for every $t \in T$. As the arbitrary solutions $p(t)$ of $(5.3)$ are uniquely determined by their value $p\left(t_{0}\right)$ at $t=t_{0}$, it follows that for every solution $p(t)$ of (5.3) on $T$ with $p\left(t_{0}\right)^{-}=a^{-}$and $p\left(t_{0}\right)^{+} \neq a^{+}\left(t_{0}, a^{-}\right):=p_{t_{0}, a^{-}}\left(t_{0}\right)^{+}$ there exists $t \in T$ such that $\|p(t)\|>\delta$. The assumptions remain verified upon small perturbations 
of $t_{0}$ and $a^{-}$, and an application of the implicit function theorem yields that the solution $p=p_{t_{0}, a^{-}}$ depends in a complex analytic way on $\left(t_{0}, a^{-}\right)$. This completes the proof of Lemma 5.2 .

Lemma 5.3. In the situation of Lemma 5.1, let $0<\delta<\delta_{0}, 0<\epsilon<\epsilon_{0}, C_{1} \delta^{2}+C_{2} \epsilon<\delta$, and $C_{3} \delta+C_{4} \epsilon<1$. Then there exists a unique solution $p(t)=p_{\uparrow}(t)$ and $p=p_{\downarrow}(t)$ of (5.3) on $\mathbb{R}+\mathrm{i} / \epsilon$ and $\mathbb{R}-\mathrm{i} / \epsilon$ such that $\|p(t)\| \leq \delta$ for every $t \in \mathbb{R}+\mathrm{i} / \epsilon$ and $t \in \mathbb{R}-\mathrm{i} / \epsilon$, respectively. Similar conclusions hold with $\mathbb{R} \pm \mathrm{i} / \epsilon$ replaced by a curve $\tau+\mathrm{i} \sigma(\tau)$, where $\tau \in \mathbb{R}, \sigma: \mathbb{R} \rightarrow \mathbb{R}$ is continuously differentiable with a bounded derivative, and the curve stays sufficiently far away from the origin.

Proof. We apply the variation on [8, p. 483] of Cotton's method.

Let $I=\mathbb{R}+\mathrm{i} / \epsilon$, and $\mathcal{Y}$ the set of all continuous functions $p: I \rightarrow \mathbb{C}^{2}$ such that $\left(t^{-1}, p(t)\right) \in D$ for every $t \in I$ and $t \mapsto w\left(t^{-1}, p(t)\right)$ is bounded on $I$. If the solution $p(t)$ of $(5.3)$ belongs to $\mathcal{Y}$, then we can let $I \ni \tau \rightarrow+\infty$ in the equation (5.4) for $\pm=+$ and let $I \ni \tau \rightarrow-\infty$ in (5.4) for $\pm=-$, and obtain $(5.5)$ and

$$
p(t)^{-}=\int_{-\infty}^{t} \mathrm{e}^{s-t} w\left(s^{-1}, p(s)\right)^{-} \mathrm{d} s,
$$

respectively. Conversely, if $p \in \mathcal{Y}$ satisfies (5.5) and (5.10), then it is a solution of (5.3).

Let $G$ denote the integral operator which assigns to each $p \in \mathcal{Y}$ the function $G(p): I \rightarrow \mathbb{C}^{2}$ such that, for each $t \in I, G(p)(t)^{+}$and $G(p)(t)^{-}$are equal to the right hand side of (5.5) and (5.10), respectively. Let $Y$ be the set of all continuous functions $p: I \rightarrow \mathbb{C}^{2}$ such that $\|p(t)\| \leq \delta$ for every $t \in I$. Then $Y \subset \mathcal{Y}, Y$ is a complete space with respect to the metric $d\left(p_{1}, p_{2}\right):=$ $\sup _{t \in U}\left\|p_{1}(t)-p_{2}(t)\right\|$, and estimates analogous to (5.7), (5.8), and (5.9) imply that $G(Y) \subset Y$ and $G$ is a contraction in $Y$ with contraction factor $\leq C_{3} \delta+C_{4} \epsilon<1$. This time the contraction mapping theorem yields a unique solution $p: I \rightarrow \mathbb{C}^{2}$ of $(5.3)$ such that $\|p(t)\| \leq \delta$ for every $t \in I$. This completes the proof of Lemma 5.3 .

Remark 5.4. According to Anosov [1, §4], "Every five years or so, someone "discovers" the theorem of Hadamard and Perron, proving it either by Hadamard's method or by Perron's." The theorem alluded to is the stable manifold theorem in dynamical systems, "Hadamard's method" refers to Hadamard [12], and "Perron's method" to Perron [22. However, Perron [22, p. 130] made perfectly clear that he used the method of Cotton [8]. The paper [8] seems to be little known, for no good reason.

Lemma 5.5. For each $m, n, r \in \mathbb{R}_{>0}$ such that $m>n$, let $\eta=\eta_{m, n, r}: \mathbb{R} \rightarrow \mathbb{R}$ be defined by $\eta(\tau)=\left(r^{2}-\tau^{2}\right)^{1 / 2}$ when $|\tau| \leq r m\left(1+m^{2}\right)^{-1 / 2}, \eta(\tau)=r\left(1+m^{2}\right)^{1 / 2}-m|\tau|$ when $r m(1+$ $\left.m^{2}\right)^{-1 / 2} \leq|\tau| \leq r\left(1+m^{2}\right)^{1 / 2} /(m-n)$, and $\eta(\tau)=-n|\tau|$ when $|\tau| \geq r\left(1+m^{2}\right)^{1 / 2} /(m-n)$. Let $V:=\{t \in \mathbb{C} \mid \operatorname{Im} t \geq \eta(\operatorname{Re} t)\}$, see Figure 5.1.

If $r$ is sufficiently large, then all solutions $p_{\uparrow}(t)$ in Lemma 5.3 have a common extension to a unique solution of (5.3), also denoted by $p_{\uparrow}(t)$, on the union $\mathcal{V}$ of $V$ with all their domains of definition. There exists $j \mapsto c_{j}: \mathbb{Z}_{>0} \rightarrow \mathbb{C}^{2}$, such that the solution $p(t)=p_{\uparrow}(t)$ of (5.3) on $\mathcal{V}$ has the asymptotic expansion

$$
p(t) \sim \sum_{j=1}^{\infty} t^{-j} c_{j}
$$

as $t \in \mathcal{V},|t| \rightarrow \infty$. The asymptotic expansion (5.11) can be differentiated termwise in the sense that

$$
\frac{\mathrm{d} p(t)}{\mathrm{d} t} \sim \sum_{j=1}^{\infty}-j t^{-j-1} c_{j}
$$


as $t \in \mathcal{V},|t| \rightarrow \infty$. All the coefficients $c_{j}$ are uniquely determined from the equations obtained by substituting (5.12) and (5.11) in the left and right hand side of (5.3), respectively, and using the power series expansion

$$
v\left(t^{-1}, p\right)=\sum_{i, j_{+}, j_{-} \in \mathbb{Z}_{\geq 0}} t^{-i}\left(p^{+}\right)^{j_{+}}\left(p^{-}\right)^{j_{-}} v_{i, j_{+}, j_{-}}, \quad v_{i, j_{+}, j_{-}} \in \mathbb{C}^{2} .
$$

We have

$$
c_{1}=-\left.\left(\left.\frac{\partial v(0, p)}{\partial p}\right|_{p=0}\right)^{-1} \frac{\partial v(u, 0)}{\partial u}\right|_{u=0} .
$$

Applying the complex conjugation $t \mapsto \bar{t}$, we obtain similar conclusions for the solutions $p_{\downarrow}(t)$ in Lemma 5.3 .

Proof. For each $t \in V$ we define half-lines $L_{t}^{ \pm}$emanating from $t$, as follows. If $\operatorname{Re} t \geq 0$, then $L_{t}^{+}:=t+\mathbb{R}_{\geq 0}$. If $0 \leq-\operatorname{Re} t \leq m \operatorname{Im} t$, then $L_{t}^{+}:=\left\{t+\tau-\mathrm{i}(\operatorname{Re} t / \operatorname{Im} t) \tau \mid \tau \in \mathbb{R}_{\geq 0}\right\}$. If $\operatorname{Re} t \leq 0$ and $m \operatorname{Im} t \leq-\operatorname{Re} t$, then $L_{t}^{+}:=\left\{t+\tau+\mathrm{i} m \tau \mid \tau \in \mathbb{R}_{\geq 0}\right\}$. Finally, $L_{t}^{-}:=R\left(\bar{L}_{R(t)}^{+}\right)$for every $t \in V$, where $R$ is the reflection $t \mapsto-\operatorname{Re} t+\mathrm{i} \operatorname{Im} t$. 5.1

It follows that, for every $t \in V$ and $s \in L_{t}^{ \pm},|t| \geq r$ and $|s| \geq(m-n)\left(1+m^{2}\right)^{-1 / 2}|t|$. See Figure

On the space of functions $p: V \rightarrow \mathbb{C}^{2}$ such that $p(t)=\mathrm{O}\left(t^{-1 / 2}\right)$ as $t \in V,|t| \rightarrow \infty$, we use the norm $\|p\|:=\sup _{t \in V}|t|^{1 / 2}\|p(t)\|$. Let $r>1 / \epsilon_{0}$ and $0<\delta<r^{1 / 2} \delta_{0}$, and $Z$ the space of continuous functions $p: V \rightarrow \mathbb{C}^{2}$, complex analytic on the interior of $V$, such that $\|p\| \leq \delta$. For each $p \in Z$ we define $G(p)$ as above, where the integrals in the right hand sides of (5.5) and (5.10) are over $L_{t}^{+}$and $L_{t}^{-}$, respectively. Using the Cauchy integral theorem, we obtain that $G(p)$ is a complex analytic function on the interior of $V$, with derivative equal to $\mathrm{d} G(p)(t)^{ \pm} / \mathrm{d} t=$ $\pm G(p)(t)^{ \pm}+w^{ \pm}\left(t^{-1}, p(t)\right)$. Because the velocities of the parametrizations of $L_{t}^{ \pm}$in the previous paragraph are at most $\left(1+m^{2}\right)^{1 / 2}$, we have the estimates

$$
\begin{aligned}
\left|G(p)(t)^{ \pm}\right| & \leq\left(1+m^{2}\right)^{1 / 2} \sup _{s \in L_{t}^{ \pm}}\left(C_{1} \delta^{2}+C_{2}\right)|s|^{-1} \\
\left|G\left(p_{1}\right)(t)^{ \pm}-G\left(p_{2}\right)(t)^{ \pm}\right| & \leq\left(1+m^{2}\right)^{1 / 2} \sup _{s \in L_{t}^{ \pm}}\left(C_{3}|s|^{-1 / 2}+C_{4}|s|^{-1}\right)\left\|p_{1}-p_{2}\right\||s|^{-1 / 2}
\end{aligned}
$$

for every $p, p_{1}, p_{2} \in Z$ and $t \in V$. Because $|s|^{-1} \leq(m-n)^{-1}\left(1+m^{2}\right)^{1 / 2}|t|^{-1}$ and $|s|^{-1} \leq r^{-1}$ for every $s \in L_{t}^{ \pm}$, it follows that $\|G(p)\| \leq\left(1+m^{2}\right)^{3 / 4}(m-n)^{-1 / 2}\left(C_{1} \delta^{2}+C_{2}\right) r^{-1 / 2}$ and $\| G\left(p_{1}\right)-$ $G\left(p_{2}\right)\left\|\leq\left(1+m^{2}\right)^{3 / 4}(m-n)^{-1 / 2}\left(C_{3} r^{-1 / 2}+C_{4} r^{-1}\right)\right\| p_{1}-p_{2} \|$. Therefore, if $r>1 / \epsilon_{0}, 0<\delta<r^{1 / 2} \delta_{0}$ $\left(1+m^{2}\right)^{3 / 4}(m-n)^{-1 / 2}\left(C_{1} \delta^{2}+C_{2}\right) r^{-1 / 2} \leq \delta$ and $\left(1+m^{2}\right)^{3 / 4}(m-n)^{-1 / 2}\left(C_{3} r^{-1 / 2}+C_{4} r^{-1}\right)<1$, which for any $m, n, \delta \in \mathbb{R}_{>0}$ can be arranged by taking $r$ sufficiently large, we have $G(Z) \subset Z$, and $G: Z \rightarrow Z$ is a contraction. This time the contraction theorem implies that there is a unique solution $p=p_{V}: V \rightarrow \mathbb{C}^{2}$ of $(5.3)$ such that $\|p(t)\| \leq \delta|t|^{-1 / 2}$ for every $t \in V$.

For any $a \in \mathbb{C}$, successive integrations by parts yield the asymptotic expansions

$$
\int_{t}^{\infty} \mathrm{e}^{t-s} s^{-a} \mathrm{~d} s \sim \sum_{k=0}^{\infty}(-1)^{k} \pi_{k} t^{-a-k} \text { and } \int_{-\infty}^{t} \mathrm{e}^{s-t} s^{-a} \mathrm{~d} s \sim \sum_{k=0}^{\infty} \pi_{k} t^{-a-k}
$$

for $|t| \rightarrow \infty$, where $\pi_{k}:=\prod_{j=0}^{k-1}(a+j)$. This is one of the oldest examples of asymptotic expansions which do not converge. Substituting the inequality $\left\|w\left(s^{-1}, p(s)\right)\right\| \leq C_{1}\|p(s)\|^{2}+C_{2}|s|^{-1} \leq$ $\left(C_{1} \delta^{2}+C_{2}\right)|s|^{-1}$ in the integrals in the right hand sides of $p(t)^{ \pm}=G(p)(t)^{ \pm}$, we obtain that $p(t)=\mathrm{O}\left(t^{-1}\right)$ as $t \in V,|t| \rightarrow \infty$. Substituting 


$$
p(t)=\sum_{j=1}^{N-1} t^{-j} c_{j}+\mathrm{O}\left(t^{-N}\right)
$$

for $t \in V$, the power series expansion $w(u, p)=\sum_{i, j_{+}, j_{-} \geq 0} w_{i, j_{+}, j_{-}} u^{i}\left(p^{+}\right)^{j_{+}}\left(p^{-}\right)^{j_{-}}$, and $u=t^{-1}$, $p=p(t)$ in the integrals in the right hand sides of $p(t)^{ \pm}=G(p)(t)^{ \pm}$, we obtain (5.15) with $N$ replaced by $N+1$ and a unique $c_{N} \in \mathbb{C}^{2}$. This inductive procedure yields that $p(t)$ has an asymptotic expansion of the form (5.11) for $t \in V,|t| \rightarrow \infty$. The substitution of $u=t^{-1}$ and $p=p(t)$ in the power series expansion of $v(u, p)$ implies that there exist $b_{i} \in \mathbb{C}^{2}$ such that $p^{\prime}(t)=v\left(t^{-1}, p(t)\right) \sim \sum_{i=1}^{\infty} t^{-i} b_{i}$ as $t \in V,|t| \rightarrow \infty$, when $\int_{t_{0}}^{t} p^{\prime}(s) \mathrm{d} s+p\left(t_{0}\right)=p(t) \sim \sum_{j=1}^{\infty} t^{-j} c_{j}$ yields that $b_{i}=-(i-1) c_{i-1}$ for every $i \in \mathbb{Z}_{\geq 1}$. This proves $(5.12)$ for $p=p_{V}$.

We will prove next that all solutions $p_{\uparrow}$ in Lemma 5.3 and $p_{V}$ have a common extension to a solution of $(5.3)$ on $\mathcal{V}$. The conditions in Lemma 5.3 hold if and only if

$$
\epsilon<\min \left\{\epsilon_{0}, 1 / C_{4}, 1 / 4 C_{1} C_{2}\right\} \quad \text { and } \quad \delta_{-}(\epsilon)<\delta<\min \left\{\delta_{0}, \delta_{+}(\epsilon)\right\},
$$

where $\delta_{ \pm}(\epsilon):=\left(1 \pm\left(1-4 C_{1} C_{2} \epsilon\right)^{1 / 2}\right) / 2 C_{1}$. If in the proof of Lemma 5.3 we replace $Y$ by the space $Y_{\epsilon, \delta}$ of all continuous functions $p$ on the upper half plane $U_{\epsilon}:=\{t \in \mathbb{C} \mid \operatorname{Im} t \geq 1 / \epsilon\}$, complex analytic in the interior of $U_{\epsilon}$ and with $\|p(t)\| \leq \delta$ for all $t \in U_{\epsilon}$, then $G$ is a contraction on $Y_{\epsilon, \delta}$ and therefore has a unique fixed point $p_{\epsilon, \delta} \in Y_{\epsilon, \delta}$. The Cauchy integral theorem implies that the restriction of $p_{\epsilon, \delta}$ to the interior of $U_{\epsilon}$ is a complex analytic solution of (5.3). As the restriction of $p_{\epsilon, \delta}$ to $\mathbb{R}+\mathrm{i} / \epsilon$ is a fixed point of $G: Y \rightarrow Y$, and the latter is unique, and equal to the solution $p_{\uparrow}$ in Lemma 5.3, it follows that the solution $p_{\uparrow}(t)$ in Lemma 5.3 extends to a solution $p=p_{\uparrow, \epsilon}$ of (5.3) on $U_{\epsilon}$, complex analytic on the interior of $U_{\epsilon}$, and satisfying $\|p(t)\| \leq \delta$ for every $t \in U_{\epsilon}$. Note that if $0<\epsilon^{\prime}<\epsilon$, then the conditions in Lemma 5.3 hold with $\epsilon$ replaced by $\epsilon^{\prime}$, and the restriction to $U_{\epsilon^{\prime}}$ of $p_{\uparrow, \epsilon}$ is equal to $p_{\uparrow, \epsilon^{\prime}}$.

If $p(t)$ is any solution of (5.3) on some upper half plane $U$, and $p(t) \rightarrow 0$ as $t \in U,|t| \rightarrow \infty$, then there exist $\delta, \epsilon$ as above such that $U_{\epsilon} \subset U$ and $\|p(t)\| \leq \delta$ for every $t \in U_{\epsilon}$, and therefore $\left.p\right|_{U_{\epsilon}}=p_{\uparrow, \epsilon}$. As the sector-like domain $V$ contains an upper half plane $U_{\epsilon}$, and $p_{V}(t) \rightarrow 0$ as $t \in V$, $|t| \rightarrow \infty$, it follows that $\left.\left(p_{V}\right)\right|_{U_{\epsilon}}=p_{\uparrow, \epsilon}$. This completes the proof that all solutions $p_{\uparrow}$ in Lemma 5.3 extend to a common solution $p(t)$ of (5.3) on $\mathcal{V}$, of which the restriction to $V$ is equal to $p_{V}$, and therefore $p$ has all the properties mentioned in Lemma 5.5 .

Lemma 5.6. In the situation of Lemma 5.1, let $\alpha$ denote the right lower corner in the $2 \times 2$-matrix

$$
L_{1}=\left.\frac{\partial^{2} v(u, p)}{\partial u \partial p}\right|_{u=0, p=0}+\left.\frac{\partial^{2} v(0, p)}{\partial p^{2}}\right|_{p=0} c_{1},
$$

with $c_{1}$ defined by 5.14). For $\eta, r \in \mathbb{R}_{>0}$, let

$$
R_{\eta, r}:=\left\{t \in \mathbb{C}|| t \mid \geq r \text { and }\left|\mathrm{e}^{-t} t^{\alpha}\right| \leq \eta\right\},
$$

where $t^{\alpha}=\mathrm{e}^{\alpha \log t}, \log t=\log |t|+\mathrm{i} \arg t$, and $-\pi \leq \arg t \leq \pi$. For every solution $p(t)=p_{t_{0}, a^{-}}(t)$ and $p(t)=p_{\uparrow}(t)$ of $(5.3)$ in Lemma 5.2 and Lemma 5.5, there exist $\eta, r \in \mathbb{R}_{>0}$ such that $p(t)$ extends to a solution of (5.3) on $R_{\eta, r}$, which extension is again denoted by $p_{t_{0}, a^{-}}$and $p_{\uparrow}$, respectively. There exist $j \mapsto d_{j}: \mathbb{Z}_{\geq 0} \rightarrow \mathbb{C}^{2}$ with $d_{0}=(0,1)$, and $C=C_{t_{0}, a^{-}} \in \mathbb{C}$, such that on any subdomain $\Sigma$ of $R_{\eta, r}$ on which $\operatorname{Re} t / \log |t| \rightarrow+\infty$ as $t \in \Sigma,|t| \rightarrow \infty$, we have the asymptotic expansion

$$
p(t)-p_{\uparrow}(t) \sim C \mathrm{e}^{-t} t^{\alpha} \sum_{j=0}^{\infty} t^{-j} d_{j}
$$


as $t \in \Sigma,|t| \rightarrow \infty$. The asymptotic expansion (5.18) can be differentiated termwise in the sense that

$$
p^{\prime}(t)-p_{\uparrow}^{\prime}(t) \sim C \mathrm{e}^{-t} t^{\alpha} \sum_{j=0} t^{-j}\left(-d_{j}+(\alpha-j+1) d_{j-1}\right)
$$

as $t \in S,|t| \rightarrow \infty$. All the coefficients $d_{j}$ are uniquely determined from the equations $d_{0}=(0,1)$ and $-d_{j}+(\alpha-j+1) d_{j-1}=\sum_{i=0}^{j} L_{i} d_{j-i}$ for all $j \geq 1$, where the $2 \times 2$-matrices $L_{i}$ are determined from the asymptotic expansion

$$
L(t):=\left.\frac{\partial v\left(t^{-1}, p\right)}{\partial p}\right|_{p=p_{\uparrow}(t)} \sim \sum_{i=0}^{\infty} t^{-i} L_{i}
$$

as $t \in V,|t| \rightarrow \infty$. Here

$$
L_{0}:=\left.\frac{\partial v(0, p)}{\partial p}\right|_{p=0}=\left(\begin{array}{cc}
1 & 0 \\
0 & -1
\end{array}\right)
$$

and $L_{1}$ is given by (5.16). The asymptotic expansion (5.11) for $p(t)=p_{\uparrow}(t)$ holds for $t \in \Sigma$, $|t| \rightarrow \infty$, and can be differentiated termwise there. In combination with (5.18), (5.19), it follows that $p(t)=p_{t_{0}, a^{-}}(t)$ has the same asymptotic expansions (5.11), (5.12) for $t \in \Sigma,|t| \rightarrow \infty$.

The complex number $C=C_{t_{0}, a^{-}}$, determined by

$$
\lim _{t \in \Sigma,|t| \rightarrow \infty} \mathrm{e}^{t} t^{-\alpha}\left(p(t)-p_{\uparrow}(t)\right)=(0, C),
$$

depends in a complex analytic way on $\left(t_{0}, a^{-}\right)$. Conversely, for every $C \in \mathbb{C}$ there exist $\eta, r \in \mathbb{R}_{>0}$ and a unique solution $p=p_{C}: R_{\eta, r} \rightarrow \mathbb{C}^{2}$ of (5.3) such that (5.18) holds, where $p_{C}(t)$ depends in a complex analytic way on $C$. If we choose $t_{0}$ such that $\left\|p\left(t_{0}\right)\right\|$ is sufficiently small, then $p=p_{t_{0}, a^{-}}$with $a^{-}=p\left(t_{0}\right)^{-}$, and $a^{-} \mapsto C_{t_{0}, a^{-}}$is a complex analytic diffeomorphism from its open domain of definition onto an open subset of $\mathbb{C}$. In particular we have $p(t) \equiv p_{\uparrow}(t)$ if and only if $C=0$ if and only if there exists a sequence $t_{j}$ in $\mathbb{C}$ such that $\left|\arg \left(t_{j}\right)\right|<\pi, \mathrm{e}^{-t_{j}} t_{j}{ }^{\alpha} \rightarrow 0$ and $\mathrm{e}^{t_{j}} t_{j}{ }^{-\alpha}\left(p\left(t_{j}\right)-p_{\uparrow}\left(t_{j}\right)\right) \rightarrow 0$ as $j \rightarrow \infty$.

Finally, for every $C \in \mathbb{C}$ and $\varepsilon>0$ there exist $\eta, r \in \mathbb{R}_{>0}$ such that

$$
\left\|\mathrm{e}^{t} t^{-\alpha}\left(p_{C}(t)-p_{\uparrow}(t)\right)-(0, C)\right\| \leq \varepsilon
$$

for every $t \in R_{\eta, r}$. In particular, if $C \neq 0$ and we choose $0<\varepsilon<|C|$, then $p_{C}(t)$ is bounded away from zero on the set of all $t \in \mathbb{C}$ such that $\left|\mathrm{e}^{-t} t^{\alpha}\right|=\eta,|t| \geq r$, and $\operatorname{Im} t>0$, the part of the boundary of $V_{\eta, r}$ in the upper half plane and sufficiently far away from the origin. A similar statement holds in the lower half plane with $p_{\uparrow}(t)$ replaced by $p_{\downarrow}(t)$.

Proof. Let $W$ be a suitable subdomain of $V$ and $p: W \rightarrow \mathbb{C}^{2}$ a solution of $(5.3)$. Then $y(t):=$ $p(t)-p_{\uparrow}(t)$ is a solution of the differential equation

$$
\mathrm{d} y / \mathrm{d} t=f\left(t^{-1}, y\right):=v\left(t^{-1}, p_{\uparrow}(t)+y\right)-v\left(t^{-1}, p_{\uparrow}(t)\right),
$$

where $(t, y) \mapsto f\left(t^{-1}, y\right)$ is a $\mathbb{C}^{2}$-valued complex analytic function on $W \times B$, if $B:=\left\{y \in \mathbb{C}^{2} \mid\right.$ $\left.\|y\|<\delta_{0}^{\prime}\right\}$, where $\delta_{0}^{\prime}:=\delta_{0}-\sup _{t \in W}\left\|p_{\uparrow}(t)\right\| \geq \delta_{0}-\delta r^{-1 / 2}$. Furthermore,

$$
f\left(t^{-1}, y\right) \sim \sum_{j=0}^{\infty} t^{-j} f_{j}(y)
$$

for $t \in V,|t| \rightarrow \infty$, where each of the functions $f_{j}$ is complex analytic on $B$. We have $f\left(t^{-1}, 0\right) \equiv 0$, hence $f_{j}(0)=0$ for every $j$. It follows from $(5.20)$ that $\partial f_{j}(y) /\left.\partial y\right|_{y=0}=L_{j}$ for every $j \in \mathbb{Z}_{\geq 0}$. All aforementioned asymptotic expansions are uniform in $y \in B$, and can be differentiated termwise, arbitrarily often, with respect to $t, y^{+}$, and $y^{-}$. 
If $A(t)=\sum_{j=0}^{N-1} t^{-j} A_{j}$, where the $A_{j}$ are $2 \times 2$-matrices with $A_{0}=1$, then the substitution $y=A(t) z$ transforms the linear differential equation $\mathrm{d} y / \mathrm{d} t=L(t) y$ into the linear equation $\mathrm{d} z / \mathrm{d} t=M(t) z$, where $L(t) A(t)=A^{\prime}(t)+A(t) M(t)$. It follows that $M(t) \sim \sum_{k=0}^{\infty} t^{-k} M_{k}$, where, for each $l \in \mathbb{Z}_{\geq 0}$

$$
\sum_{j+k=l} L_{j} A_{k}=(1-l) A_{l-1}+\sum_{j+k=l} A_{j} M_{k}
$$

Here $A_{j}=0$ when $j<0$ or $j \geq N$. It follows that $M_{0}=L_{0}$ as in (5.21), when the linear mapping $A_{l} \mapsto L_{0} A_{l}-A_{l} M_{0}$ is surjective from the space of all $2 \times 2$-matrices $A_{l}$ onto the space of all antidiagonal $2 \times 2$-matrices, with kernel equal to the space of all diagonal $2 \times 2$-matrices. It follows by induction on $l \leq N-1$ that, given the $A_{j}$ and $M_{j}$ for $j \leq l-1$, there is a unique antidiagonal matrix $A_{l}$ such that the matrix $M_{l}$ is diagonal. In other words, with the substitution $y=A(t) z$ the differential equation (5.23) is equivalent to the differential equation

$$
\mathrm{d} z / \mathrm{d} t=g\left(t^{-1}, z\right):=A(t)^{-1} f\left(t^{-1}, A(t) z\right)-A(t)^{-1} A^{\prime}(t) z,
$$

where $g\left(t^{-1}, z\right)$ has an asymptotic expansion for $|t| \rightarrow \infty$ of the same nature as $f\left(t^{-1}, y\right)$,

$$
\begin{aligned}
g\left(t^{-1}, z\right) & =\sum_{j=0}^{N-1} t^{-j} M_{j} z+h\left(t^{-1}, z\right), \\
h\left(t^{-1}, z\right) & =\mathrm{O}\left(|t|^{-N}\|z\|\right)+\mathrm{O}\left(\|z\|^{2}\right), \quad \text { and } \\
\frac{\partial h\left(t^{-1}, z\right)}{\partial z} & =\mathrm{O}\left(|t|^{-N}\right)+\mathrm{O}(\|z\|)
\end{aligned}
$$

as $t \in V$ and $|t| \rightarrow \infty$, where $M_{j}$ is a diagonal $2 \times 2$-matrix for each $0 \leq j \leq N-1$. We have $M_{0}=L_{0}$ is as in (5.21), and $M_{1}$ is equal to the diagonal part of the $2 \times 2$-matrix $L_{1}$ in (5.16).

Let $\mu_{j}^{+}$and $\mu_{j}^{-}$denote the left upper and right lower corner of the diagonal matrix $M_{j}$, where $\mu_{0}^{ \pm}= \pm 1$ and $\mu_{1}^{-}=\alpha$. Write

$$
\begin{aligned}
\Theta^{ \pm}(t) & :=\mathrm{e}^{ \pm t} t^{\mu_{1}^{ \pm}} \theta^{ \pm}(t), \quad \text { where } \\
\theta^{ \pm}(t) & :=\exp \left(\sum_{j=2}^{N-1} \mu_{j}^{ \pm} t^{1-j} /(1-j)\right) .
\end{aligned}
$$

Note that 5.29 implies that $\Theta^{ \pm}(t) \sim \mathrm{e}^{ \pm t} t^{\mu_{1}^{ \pm}}$for large $|t|$. The solutions $\zeta(t)$ of the homogeneous linear differential equation $\frac{\mathrm{d} \zeta}{\mathrm{d} t}=\sum_{j=0}^{N-1} t^{-j} M_{j} \zeta$ are given by $\zeta(t)^{ \pm}=\Theta^{ \pm}(t) \Theta^{ \pm}(\tau)^{-1} \zeta(\tau)^{ \pm}$. Substituting (5.26) in (5.25), we obtain an inhomogeneous linear differential equation for $z$ with $h\left(t^{-1}, z(t)\right)$ as the inhomogeneous term, when Lagrange's method of variation of constants yields the integral equations

$$
z(t)^{ \pm}=\frac{\Theta^{ \pm}(t)}{\Theta^{ \pm}(\tau)} z(\tau)^{ \pm}+\int_{\tau}^{t} \frac{\Theta^{ \pm}(t)}{\Theta^{ \pm}(s)} h\left(s^{-1}, z(s)\right)^{ \pm} \mathrm{d} s .
$$

The reasoning leading to $(5.5)$ and $(5.6)$ this time leads to the conclusion that $z(t)$ is a uniformly small solution of (5.25) on $T:=t_{0}+\mathbb{R}_{\geq 0}$ such that $z\left(t_{0}\right)^{-}=b^{-}$, if and only if $z(t)$ is a uniformly small continuous function which satisfies the integral equations

$$
z(t)^{+}=H(z)(t)^{+}:=-\int_{t}^{+\infty} \frac{\Theta^{+}(t)}{\Theta^{+}(s)} h\left(s^{-1}, z(s)\right)^{+} \mathrm{d} s
$$

and

$$
z(t)^{-}=H(z)(t)^{-}:=\frac{\Theta^{-}(t)}{\Theta^{-}\left(t_{0}\right)} b_{27}^{-}+\int_{t_{0}}^{t} \frac{\Theta^{-}(t)}{\Theta^{-}(s)} h\left(s^{-1}, z(s)\right)^{-} \mathrm{d} s .
$$


Assume that $N>1, \gamma>\left|b^{-} / \Theta^{-}\left(t_{0}\right)\right|$. In the next paragraphs we will prove that, if $\sup _{t \in T}\left|t^{-1}\right|$ and $\sup _{t \in T}\left|\mathrm{e}^{-t} t^{\alpha}\right|$ are sufficiently small, then $H$ is a contraction in the set $\mathcal{Z}$ of all continuous functions $z: T \rightarrow \mathbb{C}^{2}$ such that $\|z\|:=\sup _{t \in T}\left|\mathrm{e}^{t} t^{-\alpha}\right| \| z(t \| \leq \gamma$, with respect to the metric $\left(z_{1}, z_{2}\right) \mapsto\left\|z_{1}-z_{2}\right\|$.

If in the integrand in (5.30) we substitute $s=t+\tau, \tau \in \mathbb{R}_{\geq 0}$, then (5.27) implies that $\left|\mathrm{e}^{t} t^{-\alpha}\right|$ times the absolute value of the integrand is estimated from above by a uniform constant times

$$
\mathrm{e}^{-2 \tau}\left|\left(\frac{t+\tau}{t}\right)^{\alpha-\mu_{1}^{+}}\right|\left(|t+\tau|^{-N}+\left|\mathrm{e}^{-(t+\tau)}(t+\tau)^{\alpha}\right|\|z\|\right)\|z\| .
$$

In view of (5.28), a similar estimate with the last factor $\|z\|$ replaced by $\left\|z_{1}-z_{2}\right\|$ holds for $\left|\mathrm{e}^{t} t^{-\alpha}\right|\left|H\left(z_{1}\right)(t)^{+}-H\left(z_{2}\right)(t)^{+}\right|$. The integral of $\tau \mapsto \mathrm{e}^{-2 \tau}\left|\left(\frac{t+\tau}{t}\right)^{\alpha-\mu_{1}^{+}}\right|$over $\mathbb{R}_{\geq 0}$ is uniformly bounded if the distance from $T$ to the origin is bounded away from zero. As $\left|s^{-1}\right|$ and $\left|\mathrm{e}^{-s} s^{\alpha}\right|$ are uniformly small for all $s \in T$, there exists a $0<\beta<1$ such that $\left|\mathrm{e}^{t} t^{-\alpha}\right|\left|H(z)(t)^{+}\right| \leq \gamma$ and $\left|\mathrm{e}^{t} t^{-\alpha}\right|\left|H\left(z_{1}\right)(t)^{+}-H\left(z_{2}\right)(t)^{+}\right| \leq \beta\left\|z_{1}-z_{2}\right\|$ for all $z, z_{1}, z_{2} \in \mathcal{Z}$ and all $t \in T$.

In (5.31), $\left|\mathrm{e}^{t} t^{-\alpha}\right|$ times the absolute value of the integrand is estimated from above by a uniform constant times $\left(|s|^{-N}+\left|\mathrm{e}^{-s} s^{\alpha}\right|\right)\|z\|$. A similar estimate, with the last factor $\|z\|$ replaced by $\left\|z_{1}-z_{2}\right\|$, holds for the integrand in the integral formula for $\left|\mathrm{e}^{t} t^{-\alpha}\right|\left|H\left(z_{1}\right)(t)^{-}-H\left(z_{2}\right)(t)^{-}\right|$. Let $N>1$ and $\gamma>\left|b^{-} / \Theta^{-}\left(t_{0}\right)\right|$. As $\left|s^{-1}\right|$ and $\left|\mathrm{e}^{-s} s^{\alpha}\right|$ are uniformly small for all $s \in T$, there exists a $0<\beta<1$ such that $\left|\mathrm{e}^{t} t^{-\alpha}\right|\left|H(z)(t)^{-}\right| \leq \gamma$ and $\left|\mathrm{e}^{t} t^{-\alpha}\right|\left|H\left(z_{1}\right)(t)^{-}-H\left(z_{2}\right)(t)^{-}\right| \leq \beta\left\|z_{1}-z_{2}\right\|$ for all $z, z_{1}, z_{2} \in \mathcal{Z}$ and all $t \in T$. This completes the proof that $H(\mathcal{Z}) \subset \mathcal{Z}$ and $H: \mathcal{Z} \rightarrow \mathcal{Z}$ is a contraction with respect to the metric $\left(z_{1}, z_{2}\right) \mapsto \sup _{t \in T}\left|\mathrm{e}^{t} t^{-\alpha}\right|\left\|z_{1}(t)-z_{2}(t)\right\|$. Because $\mathcal{Z}$ is complete with respect to this metric, it follows that there is a unique solution $z=z_{t_{0}, b^{-}}: T \rightarrow \mathbb{C}^{2}$ of (5.25) such that $z\left(t_{0}\right)^{-}=b^{-}$and $\|z(t)\| \leq \gamma\left|\mathrm{e}^{-t} t^{\alpha}\right|$ for every $t \in T$. Because the latter estimate implies that $\|z(t)\|$ is uniformly small, the solution $p(t)=p_{\uparrow}(t)+A(t) z(t)$ of (5.3) is uniformly small on $T$, and therefore equal to the solution $p_{t_{0}, a^{-}}(t)$ in ii), with $a^{-}=\left(p_{\uparrow}\left(t_{0}\right)^{-}+A\left(t_{0}\right) z_{t_{0}, b^{-}}\left(t_{0}\right)\right)^{-}$ and $b^{-}=A\left(t_{0}\right)^{-1}\left(p_{t_{0}, a^{-}}\left(t_{0}\right)-p_{\uparrow}\left(t_{0}\right)\right)^{-}$. The assumptions remain valid upon small perturbations of $t_{0}$ and $b^{-}$, and an application of the implicit function theorem yields that the solution $z=$ $z_{t_{0}, b^{-}}$depends in a complex analytic way on $\left(t_{0}, b^{-}\right)$, when $b^{-} \mapsto a^{-}$is a local complex analytic diffeomorphism depending in a complex analytic way on $t_{0}$.

The estimates in the previous paragraph imply that the integral $\int_{t_{0}}^{\infty} \Theta^{-}(s)^{-1} h\left(s^{-1}, z(s)\right) \mathrm{d} s$ is absolutely convergent, when (5.31) implies that $\Theta^{-}(t)^{-1} z(t)^{-}$converges to

$$
C:=\Theta^{-}\left(t_{0}\right) b^{-}+\int_{t_{0}}^{\infty} \Theta^{-}(s)^{-1} h\left(s^{-1}, z(s)\right) \mathrm{d} s
$$

as $t=t_{0}+\tau, \tau \rightarrow+\infty$. Because $\Theta^{-}(t) \sim \mathrm{e}^{-t} t^{\alpha}$, the function $\mathrm{e}^{t} t^{-\alpha} z(t)^{-}$has the same limit. The previous estimates for $z(t)^{+}=H(z)(t)^{+}$yield that $\mathrm{e}^{t} t^{-\alpha} z(t)^{+}$converges to zero, and it follows that $\mathrm{e}^{t} t^{-\alpha} z_{t_{0}, b^{-}}(t)$ converges to the vector $(0, C) \in \mathbb{C}^{2}$ which depends in a complex analytic way on $\left(t_{0}, b^{-}\right)$and on $\left(t_{0}, a^{-}\right)$. Because $A(t)$ converges to the identity matrix, also $\mathrm{e}^{t} t^{-\alpha}\left(p_{t_{0}, a^{-}}(t)-\right.$ $\left.p_{\uparrow}(t)\right)=A(t)\left(\mathrm{e}^{t} t^{-\alpha} z_{t_{0}, b^{-}}(t)\right)$ converges to $\left(0, C_{t_{0}, a^{-}}\right)$as $t=t_{0}+\tau, \tau \rightarrow+\infty$.

As $b^{-}=z\left(t_{0}\right)^{-}$, and the previous remains true if we replace $t_{0}$ by any $t \in T=t_{0}+\mathbb{R}_{\geq 0}$, the equation (5.31) implies the integral equation

$$
z(t)^{-}=\mathcal{H}(z)(t)^{-}:=\Theta^{-}(t)\left(C-\int_{t}^{\infty} \Theta^{-}(s)^{-1} h\left(s^{-1}, z(s)\right)^{-} \mathrm{d} s\right) .
$$

If $\mathcal{H}(z)(t)^{+}:=H(z)(t)^{+}$, then then for every $C \in \mathbb{C}$ the integral operator $\mathcal{H}=\mathcal{H}_{C}$ is a contraction in $\mathcal{Z}$, if $\gamma>|C|$ and the numbers $\sup _{t \in T}\left|t^{-1}\right|$ and $\sup _{t \in T}\left|\mathrm{e}^{-t} t^{\alpha}\right|$ are sufficiently small. The unique fixed point $z=z_{C}$ of $\mathcal{H}_{C}$ is equal to the unique solution $z(t)$ of $(5.25)$ such that $\sup _{t \in T}\left|\mathrm{e}^{t} t^{-\alpha}\right|\|z(t)\| \leq \gamma$, and $\mathrm{e}^{t} t^{\alpha} z(t) \rightarrow(0, l)$ as $t \in T,|t| \rightarrow \infty$. 
Let $R=R_{\eta, r}$ be as in (5.17). If $\gamma>|C|$ and $\eta, r^{-1}$ are sufficiently small, then the integral operator $\mathcal{H}$ in the previous paragraph defines a contraction in the complete space of all continuous functions $z: R \cap V \rightarrow \mathbb{C}^{2}$, complex analytic in the interior of $R$, such that $\left|\mathrm{e}^{t} t^{-\alpha}\right|\|z(t)\| \leq \gamma$ for every $t \in R \cap V$, with respect to the metric $\left(z_{1}, z_{2}\right) \mapsto \sup _{t \in R \cap V}\left|\mathrm{e}^{t} t^{-\alpha}\right|\left\|z_{1}(t)-z_{2}(t)\right\|$. For every $t_{0} \in R \cap V$ the restriction of $z$ to $T:=t_{0}+\mathbb{R}_{>0}$ is equal to the unique fixed point $z_{C}$ of the integral operator $\mathcal{H}: \mathcal{Z} \rightarrow \mathcal{Z}$ in the previous paragraph, and therefore $z_{C}: T \rightarrow \mathbb{C}^{2}$ extends to a solution of (5.25) on the much larger domain $R \cap V$, which is denoted by the same letter and satisfies $\sup _{t \in R \cap V}\left|\mathrm{e}^{t} t^{-\alpha}\right|\|z(t)\| \leq \gamma$.

Substituting an asymptotic expansion $z(t) \sim \mathrm{e}^{-t} t^{\alpha} \sum_{j=0}^{k-1} t^{-j} e_{j}$ for $t \in \Sigma,|t| \rightarrow \infty$ in the right hand side of $z(t)=\mathcal{H}(z)(t)$ yields a similar expansion with $k$ replaced by $k+1$, which procedure stops at $k=N-1$. In the induction step it is used that in $\Sigma$ we have $\|z(t)\|=\mathrm{O}\left(\left|\mathrm{e}^{t} t^{\alpha}\right|\right)=\mathrm{O}\left(|t|^{-M}\right)$ for every $M>0$. This leads to an asymptotic expansion $y(t) \sim C \mathrm{e}^{-t} t^{\alpha} \sum_{j=0}^{N-1} t^{-j} d_{j}$, where the $d_{j}$ satisfy the equations $d_{0}=(0,1)$ and $-d_{j}+(\alpha-j+1) d_{j-1}=\sum_{i=0}^{j} L_{i} d_{j-i}$ for $0 \leq j \leq N-1$. For $j=1$ we have $-d_{1}+\alpha d_{0}=L_{0} d_{1}+L_{1} d_{0}$, which implies that $\left(L_{1}-\alpha\right)(0,1)$ is in the image $\mathbb{C} \times\{0\}$ of $L_{0}+1$, and we recover that $\alpha$ has to be equal to the right lower corner of $L_{1}$. If $j \geq 1$, then the equation $-d_{j}+(\alpha-j+1) d_{j-1}=L_{0} d_{j}+\sum_{i=1}^{j} L_{i} d_{j-i}$ determines $d_{j}$ only modulo the kernel $\{0\} \times \mathbb{C}$ of $L_{0}+1$, but the equation with $j$ replaced by $j+1$ implies that $\left(L_{1}-\alpha+j\right) d_{j}+\sum_{i=2}^{j} L_{i} d_{j-i}$ belongs to the image $\mathbb{C} \times\{0\}$ of $L_{0}+1$. As the right lower corner of $L_{1}-\alpha+j$ is equal to $j \neq 0$, the space $\left(L_{1}-\alpha+j\right)(\{0\} \times \mathbb{C})$ is transversal to $\mathbb{C} \times\{0\}$, and it follows that $d_{j}$ is uniquely determined by the $j$-th equation and the $(j+1)$-st equation. Because we can take $N$ arbitrarily large, and the $d_{j}, 0 \leq j \leq N-1$ do not depend on $N$, this implies (5.18), where (5.19) follows from a substitution of (5.19) in $y^{\prime}(t)=f\left(t^{-1}, y(t)\right), y(t)=p(t)-p_{\uparrow}(t)$.

This completes the proof of the lemma in the upper half plane, where $(5.22)$ follows from the aforementioned estimates for the integrals $\int_{t}^{\infty} \Theta^{ \pm}(s)^{-1} h\left(s^{-1}, z(s)\right)^{ \pm} \mathrm{d} s$. In turn (5.22), in combination with $p_{\uparrow}(t)=\mathrm{O}\left(t^{-1}\right)$, implies that $p_{C}(t)$ is bounded away from zero on the part of the boundary of $V_{\eta, r}$ in the upper half plane and sufficiently far away from the origin. The statements regarding the behavior in the lower half plane follow by replacing $p_{\uparrow}(t)$ by $p_{\downarrow}(t)$. Note that in the lower half plane $p_{\uparrow}(t)$ is equal to one of the solutions $p_{t_{0}, a^{-}}$, and therefore $p_{\uparrow}(t)$ extends from $V$ to a domain of the form $V \cup R_{\eta, r}$.

Remark 5.7. Because $p_{\downarrow}(t)$ is a solution of $(5.3)$ as in Lemma 5.2 , it follows from Lemma 5.6 that there is a unique constant $S \in \mathbb{C}$ such that

$$
p_{\downarrow}(t)-p_{\uparrow}(t) \sim \mathrm{e}^{-t} t^{\alpha}(0, S)
$$

as Ret $t \log |t| \rightarrow \infty,|t| \rightarrow \infty$, where $S=0$ implies that $p_{\downarrow}(t)=p_{\uparrow}(t)$ for all $t \in R_{\eta, r}$ with $\eta$ and $1 / r$ sufficiently small. Because this is a nonlinear analogue of the phenomena described by Stokes [26], we follow Costin [5, Th. 1] in calling $S$ the Stokes constant pertaining to the comparison in the right half plane of the solutions $p_{\downarrow}(t)$ and $p_{\uparrow}$ of $(5.3)$. Generically the Stokes constant is nonzero. This happens in particular for the Boutroux-Painlevé system, see Lemma 5.14 below.

Furthermore, for every solution $p(t)=p_{t_{0}, a^{-}}(t)$ of $(5.3)$ in Lemma 5.2 there are unique $C_{\uparrow}, C_{\downarrow} \in$ $\mathbb{C}$ such that

$$
p(t)-p_{\uparrow}(t) \sim \mathrm{e}^{-t} t^{\alpha}\left(0, C_{\uparrow}\right) \quad \text { and } \quad p(t)-p_{\downarrow}(t) \sim \mathrm{e}^{-t} t^{\alpha}\left(0, C_{\downarrow}\right)
$$

as $\operatorname{Re} t / \log |t| \rightarrow \infty,|t| \rightarrow \infty$, where conversely the solution $p(t)$ of $(5.3)$ is uniquely by any of the two asymptotic identities in 5.35 . Because $\left(p(t)-p_{\uparrow}(t)\right)-\left(p(t)-p_{\downarrow}(t)\right)=p_{\downarrow}(t)-p_{\uparrow}(t)$, it follows that

$$
C_{\uparrow}-C_{\downarrow}=S .
$$

Conversely, every complex number occurs as $C_{\uparrow}$ in $(5.35)$ for a unique solution $p(t)$ of $(5.3)$ in Lemma 5.2 , The following statements are equivalent: 
i) $C_{\downarrow}=C_{\uparrow}$ for some solution $p(t)$ of $(5.3)$ in Lemma 5.2 ,

ii) $S=0$.

iii) $C_{\downarrow}=C_{\uparrow}$ for every solution $p(t)$ of $(5.3)$ in Lemma 5.2.

iv) $p_{\downarrow}(t)=p_{\uparrow}(t)$ for all $t$ in a domain of the form $R_{\eta, r}$.

Recall the definition of $\mu_{1}^{+}$as the upper left corner of the matrix $L_{1}$ in (5.16). In analogy with (5.34), the substitution $t \mapsto-t$ leads to the existence of a unique constant $S_{-} \in \mathbb{C}$, the Stokes constant pertaining to the comparison of the solutions $p_{\downarrow}(t)$ and $p_{\uparrow}(t)$ in the left half plane, such that

$$
p_{\downarrow}(t)-p_{\uparrow}(t) \sim \mathrm{e}^{t} t^{\mu_{1}^{+}}\left(S_{-}, 0\right)
$$

as $\operatorname{Re} t / \log |t| \rightarrow-\infty,|t| \rightarrow \infty$. Here $S_{-}=0$ implies that $p_{\downarrow}(t)=p_{\uparrow}(t)$ for all $t \in-R_{\eta, r}$ with $\eta$ and $1 / r$ sufficiently small. We have $S_{-}=S=0$ if and only if $p_{\uparrow}(t)$ and $p_{\downarrow}(t)$ have a common extension to a single-valued solution $p(t)$ of 5.3 on a neighborhood of $t=\infty$ such that $p(t) \rightarrow 0$ as $t \rightarrow \infty$.

Write $\tau(t)=\mathrm{e}^{-t} t^{\alpha}$, and let

$$
p_{\text {formal }}(t)=\sum_{h, i \in \mathbb{Z}_{\geq 0}} \tau(t)^{h} t^{-i} p_{h, i}
$$

be a formal power series in $\tau(t)$ and $t^{-1}$, with coefficients $p_{h, i} \in \mathbb{C}^{2}$. The termwise derivative of the right hand side of (5.38), where $\mathrm{d}\left(\tau(t)^{h} t^{-i}\right) / \mathrm{d} t=-h \tau(t)^{h} t^{-i}+(h \alpha-i) \tau(t)^{h} t^{-i-1}$, is a formal power series in $\tau(t)$ and $t^{-1}$, which by definition is the derivative of $p_{\text {formal }}(t)$ with respect to $t$. Substitution of $p_{\text {formal }}(t)$ in the expansion (5.13) yields a formal power series in $\tau(t)$ and $t^{-1}$, which by definition is $v\left(t^{-1}, p_{\text {formal }}(t)\right)$. The formal solutions $p(t)=p_{\text {formal }}(t)$ of the differential equation (5.3) are obtained by equating the coefficients of $\tau(t)^{h} t^{-i}$.

Let $T$ be an unbounded subdomain in the complex upper half plane of the domain $R_{\eta, r}$ in Lemma 5.6, and assume that there exist strictly positive constants $C_{1}, C_{2}, \epsilon_{1}, \epsilon_{2}$ such that

$$
C_{1}|t|^{-\epsilon_{1}} \leq|\tau(t)| \leq C_{2}|t|^{-\epsilon_{2}}
$$

for every $t \in T$. In such a domain any formal series (5.38) is an asymptotic series. The proof of Lemma 5.6 yields that the solutions $p_{C}(t)$ in Lemma 5.6 have a formal series $p_{\text {formal, } C}(t)$ as their asymptotic expansion in $T$, in the sense that for every $N$ there exists an $m$ such that $p_{C}(t)-$ $\sum_{h<m, i<m} \tau(t)^{h} t^{-i} p_{h, i}=\mathrm{O}\left(t^{-N}\right)$ as $t \in T,|t| \rightarrow \infty$. The formal series $p_{\text {formal, } C}(t)$ is a formal series solution of the differential equation (5.3) such that $p_{0,0}=0$ and $p_{0,1}=(0, C)$. As will be verified in the proof of Lemma 5.8 below, a formal series solution (5.38) of (5.3) is uniquely determined by the initial consitions $p_{0,0}=0, p_{0,1}=(0, C)$, and $p_{h, i}=C^{h} c_{h, i}$ for uniquely determined universal coefficients $c_{h, i} \in \mathbb{C}^{2}$. This is the formal solution in O. and R. Costin [7, (4), (5)] for $n=2$ and $\mathbf{C}=(0, C)$, where the latter implies that only the terms with $\mathbf{k}=(0, h)$ appear in loc. cit. The proof of Lemma 5.8 also yields an independent verification of the existence of the formal series solution of $(5.3)$ such that $p_{0,0}=0$ and $p_{0,1}=(0, C)$.

In the subdomains $\Sigma$ in Lemma 5.6, the function $\tau(t)$ is of smaller order than $t^{-i}$ for every $i \in \mathbb{Z}_{\geq 0}$, when (5.38) only is an asymptotic series in the above sense if all terms with $h>0$ are deleted, and $p_{\text {formal, } C}(t)$ reduces to the right hand side of (5.11), which is independent of $C$. For $p_{C}(t)-p_{\uparrow}(t)$ we have the asymptotic expansion (5.18) in $\Sigma$, where the right hand side is equal to (5.38) with only the terms with $h=1$ retained.

If $U$ is an unbounded subdomain of $R_{\eta, r}$ in the upper half plane on which $\tau(t) \rightarrow 0$ and $t^{-i}=$ $\mathrm{o}(\tau(t))$ as $t \in U,|t| \rightarrow \infty$, then $p_{\text {formal, } C}(t)$ is only an asymptotic series for $t \in U,|t| \rightarrow \infty$ if all terms with $i>0$ are deleted, when $p_{C}(t)$ is asymptotically equal to this asymptotic series for $t \in U,|t| \rightarrow \infty$. For $t$ on the boundary of $R_{\eta, r}$ and $|t| \geq r$, the absolute value of $\tau(t)$ is equal to the constant $\eta, p_{\text {formal }, C}(t)$ is not an asymptotic series in the above sense, and we only have 
the estimate 5.22 . As $T$ is a transitional region between the subdomains $S$ on the one hand and the subdomains $U$ and the boundary of $R_{\eta, r}$ on the other, the formal series $p_{\text {formal, } C}(t)$ and the asymptotic expansion $p_{C}(t) \sim p_{\text {formal, } C}(t)$ as $t \in T,|t| \rightarrow \infty$ could be called the transitional series and the transitional expansion, respectively.

Lemma 5.8 below, which follows from O. and R. Costin [7, Th. 2(i) and Sec. 6.9], yields that for each $i \in \mathbb{Z}_{>0}$ the series $(5.39)$ converges for small $|\tau|$. This allows to formulate the asymptotic expansion (5.49) for the solution $p_{C}(t)$ of (5.3). The domain of $t$ 's where the asymptotic expansion (5.49 holds extends well beyond the part $R_{\eta, r}^{\prime}$ of the boundary of $R_{\eta, r}$ where $\operatorname{Im} t \geq 0, \tau(t)=\eta$, and $|t| \geq r$. Along $R_{\eta, r}^{\prime}$, Lemma 5.6 yielded that $p_{C}(t)$ remains bounded away from zero, but did not provide an asymptotic expansion for $|t| \rightarrow \infty$.

Lemma 5.8. Let $C \in \mathbb{C}$. Then there is a unique formal solution (5.38) of (5.3) such that $p_{0,0}=0$ and $p_{1,0}=(0, C)$. For each $i \in \mathbb{Z}_{\geq 0}$ the series

$$
F_{i}(\tau)=\sum_{h=0}^{\infty} \tau^{h} p_{h, i}
$$

converges for $\tau$ in a neighborhood of 0 in $\mathbb{C}$, where the complex analytic functions $F_{i}$ satisfy

$$
\begin{gathered}
-\tau \frac{\mathrm{d} F_{0}(\tau)}{\mathrm{d} \tau}=v_{0}\left(F_{0}(\tau)\right):=v\left(0, F_{0}(\tau)\right) \text { and } \\
-\tau \frac{\mathrm{d} F_{i}(\tau)}{\mathrm{d} \tau}=P_{i}\left(F_{0}(\tau), \ldots, F_{i}(\tau)\right)-\alpha \tau \frac{\mathrm{d} F_{i-1}(\tau)}{\mathrm{d} \tau}+(i-1) F_{i-1}(\tau)
\end{gathered}
$$

for $i \in \mathbb{Z}_{>0}$. Here $P_{i}\left(F_{0}, \ldots F_{i}\right)$ denotes the coefficient of $t^{-i}$ in the expansion of

$$
v\left(t^{-1}, \sum_{j \geq 0} t^{-j} F_{j}\right)
$$

in nonnegative integral powers of $t^{-1}$; a finite sum of weighted homogeneous polynomials of degree $\leq i$ in the $F_{j}$ with $0 \leq j \leq i$, where each $F_{j}$ has the weight $j$. In particular

$$
P_{i}\left(F_{0}, \ldots, F_{i}\right)=\frac{\partial v\left(0, F_{0}\right)}{\partial F_{0}} F_{i}+Q_{i}\left(F_{0}, \ldots F_{i-1}\right),
$$

where $Q_{i}\left(F_{0}, \ldots, F_{i-1}\right)$ is a similar polynomial in the $F_{j}$ with $0 \leq j \leq i-1$. We have

$$
F_{i}(0)=p_{0, i}=c_{i},
$$

where the $c_{i}$ are the coefficients in (5.11).

Conversely, the system (5.40), (5.41) has a unique solution $F_{i}(\tau)$ which is complex analytic on a neighborhood of $\tau=0$ in $\mathbb{C}$ such that $F_{0}(0)=0$ and $F_{0}^{\prime}(0)=(0, C)$. If $j \in \mathbb{Z}_{>0}$ then, given the functions $F_{k}(\tau)$ for $0 \leq k \leq j-1$, the function $F_{j}(\tau)$ is uniquely determined by the conditions that it is a complex analytic solution on a neighborhood of $\tau=0$ of the equation (5.41) for $i=j$, and that the equation (5.41) for $i=j+1$ admits a complex analytic solution $F_{j+1}(\tau)$ on a neighborhood of $\tau=0$. If (5.39) denotes the power series expansion of $F_{i}(\tau)$, then (5.38) is the formal solution of (5.3) in the previous paragraph.

If $F_{i, C}(\tau)$ denotes the solution of (5.40), (5.41) such that $F_{0, C}(0)=0$ and $F_{0, C}(0)=(0, C)$, then $F_{i, C}(\tau)=F_{i, 1}(C \tau)$. Let $\mathcal{T}_{C}$ be the maximal domain of definition of the, possibly multi-valued, solution $F_{0}=F_{0, C}$ of (5.40). Then $\mathcal{T}_{0}=\mathbb{C}, \mathcal{T}_{C}=C^{-1} \mathcal{T}_{1}$ if $C \neq 0$, and for each $i \in \mathbb{Z}_{>0}$ the function $F_{i, C}$ extends to a, possibly multi-valued, solution of (5.41) on $\mathcal{T}_{C}$.

Proof. With the substitutions $\tau=\mathrm{e}^{-s}, F_{0}(\tau)=p_{0}(s)$, the equation 5.40 is equivalent to the autonomous limit system

$$
\frac{\mathrm{d} p_{0}(s)}{\mathrm{d} s}=v_{0}\left(p_{0}(s)\right)
$$


of the system (5.3). Because $v_{0}(0)=v(0,0)=0$ and $v_{0}{ }^{\prime}(0)=L_{0}$, an application of Lemma 5.6 with $v$ replaced by $v_{0}$, when $c_{1}=0, L_{1}=0$, and $\alpha=0$, leads to $p_{0, \uparrow}(s)=p_{0, \downarrow}(s)=0$ and a unique solution $p_{0}(s)$ of (5.44) on Re $s \geq \sigma>>0$ such that $p_{0}(s) \sim \mathrm{e}^{-s}(0, C)$ as $\operatorname{Re} s \geq \sigma>>0$, $|s| \rightarrow \infty$. The corresponding function $F_{0}(\tau)=p_{0}(s)$ is complex analytic on the punctured disc in the complex plane determined by the inequalities $0<|\tau|<\epsilon:=\mathrm{e}^{-\sigma}$, and satisfies $F_{0}(\tau) \sim \tau(0, C)$ as $\tau \rightarrow 0$. It therefore follows from the theorem on removable singularities that $F_{0}(\tau)$ extends to a unique complex analytic function on the disc $|\tau|<\epsilon$, denoted again by $F_{0}(\tau)$, where $F_{0}(\tau)$ is a solution of $(5.40)$ on the disc $|\tau|<\epsilon$ such that $F_{0}(0)=0$ and $F_{0}{ }^{\prime}(0)=(0, C)$. As the formal power series solution $\sum_{h \geq 0} \tau^{h} p_{h, 0}$ of 5.40 with $p_{0,0}=0, p_{1,0}=(0, C)$ is unique, the above complex analytic solution $F_{0}(\tau)$ is unique as well.

Let $i \in \mathbb{Z}_{>0}$. For given complex analytic functions $F_{j}, 0 \leq j \leq i-1$, in a neighborhood of the origin, (5.41) is a linear inhomogeneous differential equations of the form

$$
-\tau \frac{\mathrm{d} F_{i}}{\mathrm{~d} \tau}=\Lambda(\tau) F_{i}+G_{i}(\tau)
$$

where $\Lambda(\tau):=\partial v(0, p) /\left.\partial p\right|_{p=F_{0}(\tau)}$ and $G_{i}(\tau)$ are complex analytic functions of $\tau$ in a neighborhood of $\tau=0$. With the substitutions $\Lambda(\tau)=\sum_{k \geq 0} \tau^{k} \Lambda_{k}$, where $\Lambda_{0}=L_{0}$ as in 5.21), and $G_{i}(\tau)=$ $\sum_{k \geq 0} \tau^{k} G_{i, k}$, the formal power series $F_{i}=\sum_{k \geq 0} F_{i, k}$ is a solution of the differential equation if and only if

$$
-k F_{i, k}=L_{0} F_{i, k}+\sum_{l=1}^{k} \Lambda_{l} F_{i, k-l}+G_{i, k}
$$

for every $k \in \mathbb{Z}_{\geq 0}$. These equations determine the coefficients $F_{i, k}$ in terms of the $F_{i, j}$ with $j<k$ and $G_{i, k}$, with the exception of the equation for $k=1$, where the + part yields $F_{i, 1}^{+}=$ $-(1 / 2)\left(\Lambda_{1} F_{i, 0}+G_{i, 1}\right)^{+}$, but the resonance in the - part yields no equation for $F_{i, 1}^{-}$, but instead the solvability condition $\left(\Lambda_{1} F_{i, 0}+G_{i, 1}\right)^{-}=0$. As $F_{i, 0}=-L_{0}{ }^{-1} G_{i, 0}$, this solvability condition is equivalent to the equation $G_{i, 1}^{-}=\left(\Lambda_{1} L_{0}{ }^{-1} G_{i, 0}\right)^{-}$for the inhomogeneous term $G_{i}(\tau)$ in the differential equation. Because $G_{i, 0}=Q_{i}\left(F_{0,0}, \ldots, F_{i-1,0}\right)+(i-1) F_{i-1,0}$ is determined, the solvability condition determines $G_{i, 1}^{-}$.

For $i=1$ we have $P_{1}\left(F_{0}, F_{1}\right)=\partial_{1} v\left(0, F_{0}\right)+\partial_{2} v\left(0, F_{0}\right) F_{1}$, hence $G_{1}(\tau)=\partial_{1} v\left(0, F_{0}(\tau)\right)-$ $\alpha \tau F_{0}^{\prime}(\tau)=\partial_{1} v\left(0, F_{0}(\tau)\right)+\alpha v\left(0, F_{0}(\tau)\right)$. Therefore $G_{1,0}=\partial_{1} v(0,0)$ and $G_{1,1}$ is equal to $C$ times

$$
\left.\frac{\partial^{2} v(u, p)}{\partial u \partial p^{-}}\right|_{u=0, p=0}+\left.\alpha \frac{\partial v(0, p)}{\partial p^{-}}\right|_{p=0},
$$

because $F_{0}^{\prime}(0)$ is equal to $C$ times the second basis vector. As the minus part of the second term is equal to $-\alpha$, it follows from the definition of $\alpha$ in Lemma 5.6 that $G_{1,1}^{-}$is equal to $C$ times the lower left corner of $\partial_{2}^{2} v(0,0) L_{0}{ }^{-1} \partial_{1} v(0,0)$. On the other hand $\Lambda_{1}$ is equal to $C$ times $\partial_{2}{ }^{2} v(0,0)$ applied to the second basis vector, when the symmetry of the second order partial derivatives yields the solvability condition $G_{1,1}^{-}=\left(\Lambda_{1} L_{0}^{-1} G_{1,0}\right)^{-}$.

For $i \geq 2$ the above computations yield that $G_{i, 1}^{-}$depends in an inhomogeneous linear way on $F_{i-1,1}^{-}$with coefficient equal to $i-1 \neq 0$. Therefore the solvability condition is satisfied by a unique choice of $F_{i-1,1}^{-}$. It follows that the system $5.40,5.41$ has a unique formal solution such that $F_{0}(0)=0$ and $F_{0}^{\prime}(0)=(0, C)$, where for the unique determination of $F_{j}(\tau), j>0$, one needs (5.41) for $i=j$ and $i=j+1$.

If in a neighborhood of $\tau=0$ the functions $F_{j}(\tau)$ are complex analytic solutions of (5.40), (5.41) for $0 \leq j \leq i-1$, then (5.45) is an inhomogeneous linear differential equation with complex analytic coefficients $\Lambda(\tau)$ and $G_{i}(\tau)$, with $\tau=0$ as a regular singular point. Therefore every formal powers series solution of (5.45) is convergent. For inhomogeneous linear differential equations this theorem, 
which sounds classical, seems to be due to Gérard and Levelt [11, Lemme 4.2]. For nonlinear higher order scalar ordinary differential equations, it has been obtained by Malgrange [20, Remarque 4.1], where the proof also works for nonlinear systems near a regular singular point.

If $g(\tau)=f(C \tau)$, then $\tau g^{\prime}(\tau)=(C \tau) f^{\prime}(C \tau)$. Therefore the function $\Phi_{0}: \tau \mapsto F_{0,1}(C \tau)$ satisfies (5.40) with $\Phi_{0}(0)=0, \Phi_{0}{ }^{\prime}(0)=C$, hence $F_{0, C}(\tau)=\Phi_{0}(\tau)=F_{0,1}(C \tau)$. Furthermore, if $F_{j}(\tau)=F_{j, 1}(C \tau)$ for $0 \leq j \leq i-1$, then $\Phi_{i}: \tau \mapsto F_{i, 1}(C \tau)$ satisfies (5.41), hence $F_{i}(\tau)=$ $\Phi_{i}(\tau)=F_{i, 1}(C \tau)$. This proves $F_{i, C}(\tau)=F_{i, 1}(C \tau)$ by induction on $i$. If the coefficients $p_{h, i}$ for $C=1$ are denoted by $c_{h, i}$, then the equations $F_{i, C}(\tau)=F_{i, 1}(C \tau)$ are equivalent to the equations $p_{h, i}=C^{h} c_{h, i}$ mentioned in the text preceding Lemma 5.8 .

The last statement in Lemma 5.8 follows, by induction on $i$, from the description of $F_{i}$ as a solution of an inhomogeneous linear differential equation (5.45), of which the coefficients $\Lambda(\tau)$ and $G_{i}(\tau)$ are complex analytic functions of $\tau \in \mathcal{T}_{C}$.

Remark 5.9. It would have been more precise to define the domain $\mathcal{T}_{C}$ as the Riemann surface of the maximal solution of $(5.40)$ such that $F_{0}(0)=0, F_{0}{ }^{\prime}(0)=(0, C)$, as follows. Let $D_{0}:=$ $\left\{p \in \mathbb{C}^{2} \mid(0, p) \in D\right\}$, where $D$ is the domain of definition of the vector field $v$ in (5.3). In $M:=\mathbb{C} \times D_{0} \backslash\left\{(\tau, p) \in \mathbb{C} \times D_{0} \mid \tau=0\right.$ and $\left.v(0, p)=0\right\}$ we have the regular complex one-dimensional distribution $\mathcal{D}$ defined by the equation $\tau \mathrm{d} p+v(0, p) \mathrm{d} \tau=0$. For the local solution $F_{0}$ of $(5.40)$ with $F_{0}(0)=0$ and $F_{0}{ }^{\prime}(0)=(0, C)$, the set $I_{C, \epsilon}:=\left\{\left(\tau, F_{0}(\tau)\right)|0<| \tau \mid<\epsilon\right\}$ is an integral manifold of $\mathcal{D}$; let $I_{C}$ denote the maximal integral manifold of $\mathcal{D}$ which contains $I_{C, \epsilon}$. Then $\mathcal{T}_{C}$ is canonically identified with the Riemann surface $I_{C} \cup\{(0,0)\}$. The inverse of the projection $I_{C} \ni(\tau, p) \rightarrow \tau$ followed by the projection $(\tau, p) \mapsto p$ is the, possibly multi-valued, maximal solution $F_{0, C}$ of 5.40 mentioned in Lemma 5.8 .

Lemma 5.11 below follows from O. and R. Costin [7, Th. 2(ii)]. Our proof is different. Lemma 5.11 implies all the previous asymptotic expansions in Section 5.1, but not the explicit estimates such as in Lemma 5.2 and 5.3 .

The following Lemma 5.10 will be used in the last part of the proof of Lemma 5.11, and in Lemma 5.18 about the poles of the truncated solutions. Lemma 5.10 is a detailed version of the estimate in [7, (24)].

Lemma 5.10. Let $\tau \in \mathbb{C} \backslash\{0\}$ and $\log \tau$ a given solution $\lambda$ of the equation $\mathrm{e}^{\lambda}=\tau$. Then the solutions $t$ of the equation $\mathrm{e}^{-t} t^{\alpha}=\tau$, where $t^{\alpha}=\mathrm{e}^{\alpha \log t}, \log t=\log |t|+\mathrm{i} \arg t,|\arg t| \leq \pi$, and $|t|$ is large, form two sequences $t_{n}$, where $n \in \mathbb{Z}, n>>0$ and $n<<0$ respectively, such that

$$
t_{n}=2 \pi \mathrm{i} n+\alpha \log (2 \pi \mathrm{i} n)-\log \tau+s(u, w)
$$

as $|n| \rightarrow \infty$. Here $\log (2 \pi \mathrm{i} n):=\log (2 \pi|n|)+\mathrm{i} \operatorname{sgn}(n) \pi / 2$,

$$
u:=\frac{1}{2 \pi \mathrm{i} n}, \quad v:=\frac{\log (2 \pi \mathrm{i} n)}{2 \pi \mathrm{i} n}, \quad w:=-u \log \tau+\alpha v,
$$

and $s(u, w)$ is a convergent power series in $(u, w)$. More precisely, $s(u, w)$ is a complex analytic function of $(u, w)$ in a neighborhood of $(u, w)=(0,0)$, equal to the unique small solution $s$ of the equation

$$
s=f(u, w, s):=\alpha \log (1+w+\alpha u \log (1+u s+w))
$$

for $u$ and $w$ both small. It follows that, with the substitutions (5.47) and $\lambda:=\log \tau$,

$$
\begin{aligned}
s(u, w)= & \alpha w+\alpha^{2} u w-\alpha w^{2} / 2+\alpha^{3} u^{2} w-3 \alpha^{2} u w^{2} / 2+\alpha w^{3} / 3+\mathrm{O}\left(n^{-4}\right) \\
= & -\alpha \lambda u+\alpha^{2} v-\alpha \lambda(\alpha+\lambda / 2) u^{2}+\alpha^{2}(\alpha+\lambda) u v-\alpha^{3} v^{2} / 2 \\
& -\alpha \lambda\left(\alpha^{2}+3 \alpha \lambda / 2+\lambda^{2} / 3\right) u^{3}+\alpha^{2}\left(\alpha^{2}+3 \alpha \lambda+\lambda^{2}\right) u^{2} v \\
& -\alpha^{3}(3 \alpha / 2+\lambda) u v^{2}+\alpha^{4} v^{3} / 3+\mathrm{O}\left(n^{-4}\right) .
\end{aligned}
$$


The $t_{n}$ depend in a complex analytic way on $\log \tau, \tau \in \mathbb{C} \backslash\{0\}$. If $\tau$ runs around the origin once in the positive direction, then $t_{n}(\tau)$ moves continuously to $t_{n-1}(\tau)$. In this sense the $\tau_{n}(\tau)$ for $n>>0$ and $n<<0$ can be viewed as two multi-valued complex analytic functions of $\tau$.

Proof. The equation $\mathrm{e}^{-t} t^{\alpha}=\tau$ is equivalent to the equation $t-\alpha \log t=2 \pi \mathrm{i} n-\log \tau$ for some $n \in \mathbb{Z}$. For large $|t|, \log |t|=\mathrm{o}(|t|),|n|$ is large, $2 \pi \mathrm{i} n / t=1+o(1)$, and $t=2 \pi \mathrm{i} n(1+\mathrm{o}(1))$.

With the notation $a:=2 \pi \mathrm{i} n-\log \tau$, the equation for $t$ is equivalent to $0=\phi(t):=(t-$ a) $\left(1-(t-a)^{-1} \alpha \log t\right)$. If $\gamma:[0,1] \rightarrow \mathbb{C} \backslash\{a\}$ is a Jordan curve which winds once around $a$ in the positive direction and $|\alpha||\log s|<|s-a|$ for all $s \in \gamma([0,1])$, then the the winding number $=(2 \pi)^{-1}$ times the increase of the argument of $\phi \circ \gamma a$ is equal to one, hence the function $\phi$ has a unique zero in the interior of $\gamma$, which is simple, and therefore in view of the implicit function theorem depends in a complex analytic way on $a$. If $|a|>1$ and $|s-a|=|a|-1$, then $1 \leq|s| \geq 1,|\alpha||\log s| \leq|\alpha|(\pi+\log |s|+\pi) \leq|\alpha|(\pi+\log (2|a|-1))$ and therefore $|\alpha||\log s|<|t-a|$ for all $s$ on the circle $\gamma$ around $a$ with radius $|a|-1$ if $|\alpha|(\pi+\log (2|a|-1))<|a|-1$, which happens if $|a|$ is sufficiently large. Because $|n|>>0$ implies that $|a|=\mid 2 \pi$ i $n-\log \tau \mid>>0$, and $t-a=\alpha \log t=\alpha \log (2 \pi \mathrm{i} n)+\mathrm{o}(1)=\alpha \log a+\mathrm{o}(1)=\mathrm{o}(|a|)$, the conclusion is that every solution $t$ of the equation $t-\alpha \log t=2 \pi \mathrm{i} n-\log \tau$ such that $t=2 \pi \mathrm{i} n(1+\mathrm{o}(1))$ is equal to the unique zero of $\phi$ in the disc around $a$ with radius $|a|-1$. Therefore the solution $t=t_{n}(\tau)$ of the equation $t-\alpha \log t=2 \pi \mathrm{i} n-\log \tau$ with $|\arg t| \leq \pi$ and $|t|>>0$ is unique, depends in a complex analytic way on $\tau$, and satisfies $t=2 \pi$ i $n(1+\mathrm{o}(1))$.

If we substitute the latter estimate for $t$ in the right hand side of $t=2 \pi \mathrm{i} n-\log \tau+\alpha \log t=$ $2 \pi \mathrm{i} n-\log \tau+\alpha\left(\log (2 \pi \mathrm{i} n)+\log \left(1+(2 \pi \mathrm{i} n)^{-1}(-\log \tau+\alpha \log t)\right)\right.$, we obtain that $t=2 \pi \mathrm{i} n+$ $\alpha \log (2 \pi \mathrm{i} n)-\log \tau+s$ with $s=\mathrm{O}((\log |n|) /|n|) \rightarrow 0$ as $|n| \rightarrow \infty$. With the definitions of $u, v$, and $w$ in (5.47), $s$ satifies the equation (5.48). Because $f(0,0, s) \equiv 0$, it follows from the implicit function theorem in the complex analytic setting that there exist open neighborhoods $U, W$, and $S$ of the origin in $\mathbb{C}$ such that for each $(u, w) \in U \times W$ the equation (5.48) has a unique solution $s=s(u, w) \in S$, and that $(u, w) \mapsto s(u, w)$ is a complex analytic function on $U \times W$, with $s(0,0)=0$. Substitution of (5.47) in $s=s(u, w)$ leads to (5.46). Subsequent differentiations of $s(u, w)=f(u, w, s(u, w))$ with respect to $(u, w)$ at $(u, w)=(0,0)$ up to the order three and subsequent evaluations of the results at $(u, w)=(0,0)$ lead to the asymptotic formulas for $t_{n}(\tau)$ modulo terms of order $n^{-4}$. With the help of a formula manipulation computer program one could go on a little bit further, but the complexity of the asymptotic formulas increases rapidly with growing order.

If $\tau$ runs around the origin once in the positive direction, then $2 \pi$ i $n-\log \tau$ moves continuously to $2 \pi \mathrm{i}(n-1)-\log \tau$, and therefore $t_{n}(\tau)$ moves continuously to $t_{n-1}(\tau)$.

Lemma 5.11. Let $K$ be a compact subset of $\mathbb{C}^{2}$ such that $\{0\} \times K$ is contained in the domain of definition $D$ of the vector field $v$ in (5.3). Let $\mathcal{T}_{0}$ be a bounded subdomain of the domain $\mathcal{T}$ in Lemma 5.8, such that $F_{0}(\tau) \in K$ for every $\tau \in \mathcal{T}_{0}$. Let $V$ be a $t$-domain in the complex upper half plane where $|t|>>0$ and $\tau(t) \in \mathcal{T}_{0}$. Then the solution $p_{C}(t)$ in Lemma 5.6 extends to a, possibly multi-valued, solution $p(t)$ of (5.3) on $V$. This solution has the asymptotic expansion $p(t) \sim \sum_{i \geq 0} t^{-i} F_{i}(\tau(t))$ in the sense that, for every $m \in \mathbb{Z}_{>0}$,

$$
p(t)=\sum_{i=0}^{m-1} t^{-i} F_{i}(\tau(t))+\mathrm{O}\left(t^{-m}\right)
$$

as $t \in V,|t| \rightarrow \infty$.

Proof. Write

$$
p^{[m]}(t):=\sum_{i=0}^{m-1} t^{-i} F_{i}(\tau(t)), \quad p_{0}^{[m]}(t):=\sum_{i=0}^{m-1} t^{-i} F_{i}(0), \quad \text { and } \quad \delta(t):=p(t)-p^{[m]}(t) .
$$


According to Lemma 5.6, $p(t)-p_{\uparrow}(t) \sim C \tau(t)$ as $\tau(t) \rightarrow 0$. The expansion (5.18) and equation (5.43) imply that $p_{\uparrow}(t)-p_{0}^{[m]}(t)=\mathrm{O}\left(t^{-m}\right)$. Finally $p^{[m]}(t)-p_{0}^{[m]}(t)=F_{0}(\tau(t))-F_{0}(0)+\sum_{i=1}^{i-1} t^{-i}\left(F_{i}(\tau(t))-\right.$ $\left.F_{i}(0)\right) \sim C \tau(t)+\mathrm{O}\left(t^{-1} \tau(t)\right) \sim C \tau(t)$ as $\tau(t) \rightarrow 0$, because $F_{0}(\tau) \sim C \tau$ as $\tau \rightarrow 0$ and $\tau(t) \rightarrow 0$ implies that $t \rightarrow 0$. Therefore $y^{[m]}(t):=\left(p(t)-p_{\uparrow}(t)\right)-\left(p^{[m]}(t)-p_{0}^{[m]}(t)\right)=\mathrm{o}(\tau(t))$, as $\tau(t) \rightarrow 0$; and $\delta(t)=y^{[m]}(t)+\mathrm{O}\left(t^{-m}\right)$, hence $(5.49) \Leftrightarrow y^{[m]}(t)=\mathrm{O}\left(t^{-m}\right)$.

The differential equations (5.40) and (5.41) imply that

$$
\begin{aligned}
\frac{\mathrm{d} p^{[m]}(t)}{\mathrm{d} t}= & v\left(0, F_{0}(\tau(t))\right)+\sum_{i=1}^{m-1} t^{-i}\left[L_{0} F_{i}(\tau(t))+P_{i}\left(F_{0}(\tau(t)), \ldots, F_{i-1}(\tau(t))\right)\right] \\
& +\left.t^{-m}\left(\alpha \tau \frac{\mathrm{d} F_{m-1}(\tau)}{\mathrm{d} \tau}-(m-1) F_{m-1}(\tau)\right)\right|_{\tau=\tau(t)}=v\left(t^{-1}, p^{[m]}(t)\right)+\mathrm{O}\left(t^{-m}\right),
\end{aligned}
$$

hence

$$
\begin{aligned}
\frac{\mathrm{d} y^{[m]}(t)}{\mathrm{d} t} & =\frac{\mathrm{d} p(t)}{\mathrm{d} t}-\frac{\mathrm{d} p^{[m]}(t)}{\mathrm{d} t}-\frac{\mathrm{d}\left(p_{\uparrow}(t)-p_{0}^{[m]}(t)\right)}{\mathrm{d} t} \\
& =v\left(t^{-1}, p(t)\right)-\left(v\left(t^{-1}, p^{[m]}(t)\right)+\mathrm{O}\left(t^{-m}\right)\right)+\mathrm{O}\left(t^{-m-1}\right) \\
& =v\left(t^{-1}, p^{[m]}(t)+y^{[m]}(t)\right)-v\left(t^{-1}, p^{[m]}(t)\right)+\mathrm{O}\left(t^{-m}\right)
\end{aligned}
$$

as long as $\tau(t) \in \mathcal{T}_{0}$, where in the last identity we have used that $p(t)=p^{[m]}(t)+y^{[m]}(t)+\left(p_{\uparrow}(t)-\right.$ $\left.p_{0}^{[m]}(t)\right)=p^{[m]}(t)+y^{[m]}(t)+\mathrm{O}\left(t^{-m}\right)$.

We start with the proof for $t$ in a domain $R_{\eta, r}$ as in (5.17) with $\eta>0$ sufficiently small, when (5.49) is [7, (20)] in our situation. It follows that $\mathrm{d} y^{[m]}(t) / \mathrm{d} t=L\left(t, p^{[m]}(t)\right) y^{[m]}(t)+\mathrm{O}\left(\left\|y^{[m]}(t)\right\|^{2}\right)+$ $\mathrm{O}\left(t^{-m}\right)$, where $L(t, p):=\partial v\left(t^{-1}, p\right) / \partial p$. Because $p^{[m]}(t)=p_{\uparrow}(t)-\left(p_{\uparrow}(t)-p_{0}^{[m]}(t)\right)+p^{[m]}(t)-$ $\left.p_{0}^{[m]}(t)\right)=p_{\uparrow}(t)+\mathrm{O}\left(t^{-m}\right)+\mathrm{O}(\tau(t))$, we have $L\left(t, p^{[m]}(t)\right)=L(t)+\mathrm{O}\left(t^{-m}\right)+\mathrm{O}(\tau(t))$ with $L(t)$ as in $(5.20)$, hence

$$
\frac{\mathrm{d} y^{[m]}(t)}{\mathrm{d} t}=L(t) y^{[m]}(t)+\mathrm{O}\left(|\tau(t)|\left\|y^{[m]}(t)\right\|\right)+\mathrm{O}\left(\left\|y^{[m]}(t)\right\|^{2}\right)+\mathrm{O}\left(t^{-m}\right) .
$$

Applying a linear substitution of variables $y=A(t) z$ as in the proof of Lemma 5.6 we arrive at an integral equation $z=\mathcal{H}(z)$ for $z: t \mapsto A(t)^{-1} y^{[m]}(t)$, where $\mathcal{H}(z)(t)^{+}=H(z)(t)^{+}$and $\mathcal{H}(z)(t)^{-}$ are as in 5.30 and 5.33), respectively, $C=0$ in view of $z(t)=A(t)^{-1} y^{[m]}(t)=\mathrm{o}(\tau(t))$, and $h\left(t^{-1}, z\right)=\mathrm{O}(|\tau(t)|\|z\|)+\mathrm{O}\left(\|z\|^{2}\right)+\mathrm{O}\left(t^{-m}\right)$. If $\eta$ and $1 / r$ are sufficiently small, then $\mathcal{H}$ is a contraction on the space $\mathcal{Z}$ of all continuous functions $z: R_{\eta, r} \rightarrow \mathbb{C}^{2}$ that are complex analytic on the interior of $R_{\eta, r}$ and satisfy a uniform bound $\|z(t)\| \leq C|t|^{-m}$, where $\mathcal{H}$ is a contraction with respect to the metric $\left(z_{1}, z_{2}\right) \mapsto \sup _{t \in R_{\eta, r}}|t|^{m}\left\|z_{1}(t)-z_{2}(t)\right\|$. It follows that $\mathcal{H}$ has a unique fixed point $z$ in $\mathcal{Z}$, when $y^{[m]}(t)=A(t) z(t)$ satisfies $y^{[m]}(t)=\mathrm{O}\left(t^{-m}\right)$ when $t \in R_{\eta, r}$ and $|t| \rightarrow \infty$. This completes the proof for $t$ in a domain where $|\tau(t)|$ remains sufficiently small.

For $t$ in a subdomain of $V$ where $\tau(t):=\mathrm{e}^{-t} t^{\alpha} \in \mathcal{T}_{0}$ remains bounded away from zero, we use $\tau=\tau(t)$ instead of $t$ as the independent variable. The domain $\mathcal{T}_{0}$ can be arranged such that its points $\tau$ can be joined with points $\tau_{0}$ close to the origin by smooth paths $\gamma$ in $\mathcal{T}_{0}$, parametrized by arclength, with a uniformly bounded length, and staying at a uniform distance away from the origin. The solution $y^{[m]}(\tau)$ will be estimated along such $\gamma$. With $\tau$ along $\gamma$, we view $t=t_{n}(\tau)$, $n>>0$, as the multi-valued inverse in Lemma 5.10 of the function $t \mapsto \tau(t)$ with large $|t|$ and $0<\arg t<\pi$. Then $t=t_{n}(\tau)=2 \pi \mathrm{i} n(1+\mathrm{o}(1))$, uniformly for $\tau$ along $\gamma$. Because

$$
\frac{\mathrm{d} y^{[m]}}{\mathrm{d} \tau}=(-1+\alpha / t)^{-1} \tau^{-1} \frac{\mathrm{d} y^{[m]}}{\mathrm{d} t}=\mathrm{O}\left(\left\|y^{[m]}\right\|\right)+\mathrm{O}\left(\left|t_{n}(\tau)\right|^{-m}\right),
$$


we have

$$
\frac{\mathrm{d}\left\|y^{[m]}(\gamma(s))\right\|}{\mathrm{d} s} \leq\left\|\frac{\mathrm{d} y^{[m]}(\gamma(s))}{\mathrm{d} s}\right\| \leq\left\|\left(y^{[m]}\right)^{\prime}(\gamma(s))\right\| \leq C_{1}\left\|y^{[m]}(\gamma(s))\right\|+C_{2}\left|t_{n}(\gamma(s))\right|^{-m}
$$

for some positive constants $C_{1}$ and $C_{2}$. Or,

$$
\frac{\mathrm{d}\left\|y^{[m]}(\gamma(s))\right\|}{\mathrm{d} s}=C_{1}\left\|y^{[m]}(\gamma(s))\right\|+C_{2}\left|t_{n}(\gamma(s))\right|^{-m}-r(s)
$$

for a non-negative continuous function $r(s)$. Lagrange's variation of constants formula yields

$$
\begin{aligned}
\left\|y^{[m]}(\gamma(s))\right\| & =\mathrm{e}^{C_{1} s}\left\|y^{[m]}(\gamma(0))\right\|+\int_{s_{0}}^{s} \mathrm{e}^{C_{1}(s-\sigma)}\left(C_{2}\left|t_{n}(\gamma(\sigma))\right|^{-m}-r(\sigma)\right) \mathrm{d} \sigma \\
& \leq \mathrm{e}^{C_{1} s}\left\|y^{[m]}(\gamma(0))\right\|+C_{2} \int_{0}^{s} \mathrm{e}^{C_{1}(s-\sigma)}\left|t_{n}(\gamma(\sigma))\right|^{-m} \mathrm{~d} \sigma .
\end{aligned}
$$

Write $t=t_{n}(\gamma(s))$, where $|t|>>0$. Then, uniformly for $0 \leq \sigma \leq s, t_{n}(\gamma(\sigma))=2 \pi \mathrm{i} n(1+\mathrm{o}(1))=$ $t(1+\mathrm{o}(1))$, hence $\left|t_{n}(\gamma(\sigma))\right|^{-m}=\mathrm{O}\left(|t|^{-m}\right)$. Furthermore, because $\gamma(0)=\tau_{0}$ is small, $\left\|y^{[m]}(\gamma(0))\right\|=$ $\mathrm{O}\left(\left|t_{n}(\gamma(0))\right|^{-m}\right)=\mathrm{O}\left(|t|^{-m}\right)$. Combination of the estimates yields $y^{[m]}(\gamma(s))=\mathrm{O}\left(t^{-m}\right)$. That is, returning to $t$ as the independent variable, $y^{[m]}(t)=\mathrm{O}\left(t^{-m}\right)$.

Lemma 5.11 can be used in order to obtain asymptotic information about the large $t$ where the solution $p_{C}(t)$ becomes singular, where $\tau(t)$ is close to points $\tau$ where the solution $F_{0}(\tau)$ of $(5.40)$ becomes singular. This will be done in more detail for the Boutroux-Painlevé system in Subsection 5.2 leading to the asymptotic description in Lemma 5.18 of the poles in the domain $R_{\eta, r}$ with large $\eta$ and $r$ correspondingly large. These poles correspond to the first sequence of poles of the truncated and triply truncated solutions of the first Painlevé equation which appear beyond the boundary of the truncated domains.

5.2. Truncated and triply truncated solutions. In this subsection we collect the conclusions from Subsection 5.1 for the Painlevé equation.

Boutroux [2, §13] found a family of solutions $u(z)$ of the Boutroux-Painlevé system (2.2), depending in a complex analytic way on one complex variable, such that $u(z)$ converges to the equilibrium point $(\epsilon \mathrm{i} \sqrt{6}, 0)$ of $(5.1)$ as $t=\lambda_{+} z$ runs to infinity in the complex plane in the direction of the positive real axis. On [2, p. 346], he called these solutions truncated in the direction of the positive real axis. These solutions correspond to the solutions $p_{t_{0}, a^{-}}(t)$ and $p_{l}(t)$ described in the lemmas 5.2 and 5.6 .

In [2, §14], Boutroux found a solution $u(z)$ which converges to the equilibrium point of (5.1) as $t=\lambda^{+} z$ runs to infinity in a sector in the complex plane which does not contain the positive imaginary axis. Because such a sector contains the three orthogonal axes equal to the negative real, negative imaginary, and positive real one, he called this solution the triply truncated solution. This solution corresponds to the solution $p_{\downarrow}(t)$ described in the lemmas 5.3, 5.5, and 5.6. In the sequel we will follow Boutroux's terminology.

Remark 5.12. The proofs in Boutroux [2, $\S 13,14]$ contain gaps and errors. In the formula for $Y_{1}$ following [2, (51)] the factor $X^{-1}$ in the first and second integral should be replaced by $-X^{-2} / \sqrt{12}$ and $X^{-2} / \sqrt{12}$, respectively. A similar correction is needed in [2, (54 bis)]. In the right hand side of [2, (54)] the term $6 \sum_{k=1}^{j-1} Y_{k} Y_{j-k}$ is missing. More seriously, the inductive assumption that $Y_{k}(X) \rightarrow 0$ as $X \rightarrow \infty$ for every $k<j$ implies that the second limit relation at the bottom of [2, p. 343] is automatically satisfied, when the first one is equivalent to the condition that $Y_{j}(X) \rightarrow 0$ as $X \rightarrow \infty$. This determines $Y_{j}$ only up to the addition of a constant times $\mathrm{e}^{-\sqrt{12} X}$. Therefore the estimate $l_{j}<K l_{j-1} \bar{X}^{\alpha-1}$ on [2, p. 345] cannot be proved for the solutions $Y_{j}$ described by 
Boutroux. It is a bit surprising that Boutroux did not use the method of Cotton [8], which appeared two years earlier in the same journal as the paper of Boutroux.

Remark 5.13. On [2, p. 261], Boutroux gave a definition of truncated solutions which looks quite different from the definition in terms of the convergence to the equilibrium point of the limit system. In our notation, we understand his definition on [2, p. 261] in the following way. For every $z_{0} \in \mathbb{C} \backslash\{0\}$ and $c \in \mathbb{C}$ there is a unique solution $U(z)=U_{z_{0}, c}(z)$ of $(2.4)$ which has a pole at $z=z_{0}$ and $c$ as the coefficient of $\left(z-z_{0}\right)^{4}$ in its Laurent expansion at $z=z_{0}$. The parameter $c$ corresponds in an bijective affine manner to the position on the pole line $L_{9} \backslash L_{8}^{(1)}$ in Okamoto's space, see 3.2 . Assume that $u_{z_{0}, c}(z)$ has another pole at $z=z_{1} \neq z_{0}$. Because the vector field is regular, nonzero at and transversal to the pole line, an application of the implicit function theorem yields that for every $\left(z_{0}^{\prime}, c^{\prime}\right)$ near $\left(z_{0}, c\right)$ there is a unique $z_{1}^{\prime}=Z_{1}\left(z_{0}{ }^{\prime}, c^{\prime}\right)$ near $z_{1}$ such that the solution $u_{z_{0}{ }^{\prime}, c^{\prime}}(z)$ has a pole at $z=z_{1}{ }^{\prime}$, and the function $\left(z_{0}{ }^{\prime}, c^{\prime}\right) \mapsto Z_{1}\left(z_{0}{ }^{\prime}, c^{\prime}\right)$ is complex analytic. It seems that Boutroux considered this to be evident, as he did not provide further proof. Then, according to our interpretation of [2, p. 261], $u_{z_{0}{ }^{\prime \prime}, c^{\prime \prime}}(z)$ is a truncated solution if the function $Z_{1}\left(z_{0}{ }^{\prime}, c^{\prime}\right)$ has a complex analytic continuation for $\left(z_{0}{ }^{\prime}, c^{\prime}\right)$ on a path approaching $\left(z_{0}{ }^{\prime \prime}, c^{\prime \prime}\right)$, but that the superior limit of $\left|Z_{1}\left(z_{0}{ }^{\prime}, c^{\prime}\right)\right|$ is infinite if $\left(z_{0}^{\prime}, c^{\prime}\right) \rightarrow\left(z_{0}^{\prime \prime}, c^{\prime \prime}\right)$ along the path. We find this an interesting definition of truncated solutions. However, we did not find any statement or proof in Boutroux's paper which relates the truncated solutions as defined on [2, p. 261] with the truncated solutions defined in term of their convergence to one of the equilibrium points of the limit system. We will stay with the second definition, and view it as a challenge to find relations with the first one.

According to Remark 2.1, the substitutions $x=-2^{-3 / 5} 3^{-1 / 5} \xi, y(x)=2^{-4 / 5} 3^{-3 / 5} \eta(\xi)$ turn the first Painlevé equation (1.1) into

$$
\mathrm{d}^{2} \eta / \mathrm{d} \xi^{2}=\left(\eta^{2}-\xi\right) / 2
$$

when the substitutions $\xi=((5 / 4) t)^{4 / 5}, \eta(\xi)=\xi^{1 / 2} \pi_{1}(t)$, and $\eta^{\prime}(\xi)=\xi^{3 / 4} \pi_{2}(t)$ turn the corresponding first order system for $\left(\eta, \eta^{\prime}\right)$ into

$$
\begin{aligned}
& \mathrm{d} \pi_{1} / \mathrm{d} t=\pi_{2}-2(5 t)^{-1} \pi_{1}, \\
& \mathrm{~d} \pi_{2} / \mathrm{d} t=\left(\pi_{1}^{2}-1\right) / 2-3(5 t)^{-1} \pi_{2} .
\end{aligned}
$$

At the equilibrium point $\left(\pi_{1}, \pi_{2}\right)=(1,0)$ of the limit system for $t=\infty$, the linearization has the eigenvalues \pm 1 , and the substitution of variables $\pi_{1}=1+p^{+}+p^{-}, \pi_{2}=p^{+}-p^{-}$lead to the system (5.3) with

$$
\begin{aligned}
& v(1 / t, p)^{+}=p^{+}-1 / 5 t-p^{+} / 2 t+p^{-} / 10 t+\left(p^{+}+p^{-}\right)^{2} / 4, \\
& v(1 / t, p)^{-}=-p^{-}-1 / 5 t-p^{-} / 2 t+p^{+} / 10 t-\left(p^{+}+p^{-}\right)^{2} / 4 .
\end{aligned}
$$

It follows that the vector $c_{1}$ in (5.14) and the matrix $L_{1}$ in (5.16) are equal to

$$
c_{1}=\left(\begin{array}{c}
1 / 5 \\
-1 / 5
\end{array}\right) \quad \text { and } \quad L_{1}=\left(\begin{array}{cc}
-1 / 2 & 1 / 10 \\
1 / 10 & -1 / 2
\end{array}\right)
$$

respectively. Because $c_{1}^{+}+c_{1}^{-}=0$, the asymptotic expansion (5.11) implies that for the truncated solutions $p(t)$ we have $\pi_{1}(t)=1+\mathrm{O}\left(t^{-2}\right)$ as $t \in S,|t| \rightarrow \infty$. Furthermore, both the left upper corner and the right lower corner $\alpha$ of $L_{1}$ are equal to $-1 / 2$, and therefore $p(t)-p_{\uparrow}(t)$ is asymptotically equal to $\tau(t)=\mathrm{e}^{-t} t^{-1 / 2}$ times a series in nonnegative powers of $t^{-1}$ as $t \in \Sigma,|t| \rightarrow \infty$, see (5.18). The fact that $\operatorname{Re} \alpha<0$ implies that the large positive and negative parts of the imaginary axis are contained in the interior of the domains $R_{\eta, r}$ in (5.17). More precisely, the part in $|t| \geq r$ of the boundary of $R_{\eta, r}$ is given by the equation

$$
\cos (\arg t)=(-(1 / 2) \log |t|-\log \eta) /|t|, \quad|t| \geq r, \quad-\pi<\arg t<\pi,
$$

which is to the left of the imaginary axis if and only if $|t|>\eta^{-2}$. See Figure 5.2 . 
Because the Painlevé property implies that all solutions $y(x)$ of (1.1) are single-valued, the analytic continuation of $\left(\pi_{1}(t), \pi_{2}(t)\right)$ along the path $t \mathrm{e}^{\mathrm{i} \theta}$, where $\theta \in \mathbb{R}$ runs from 0 to $5 / 4$ times $2 \pi$, applied to the substitutions

$$
x=-2^{-3 / 5} 3^{-1 / 5}(5 t / 4)^{4 / 5}, \quad y(x)=2^{-4 / 5} 3^{-3 / 5}\left(-2^{3 / 5} 3^{1 / 5} x\right)^{1 / 2} \pi_{1}(t)
$$

leaves the solution $y(x)$ of $(1.1)$ invariant. We conclude as in (2.6) that the analytic continuation of $\left(\pi_{1}(t), \pi_{2}(t)\right)$ along the aforementioned path is equal to $\left(-\pi_{1}(t), \mathrm{i} \pi_{2}(t)\right)$. This agrees with the symmetry $\left(t, \pi_{1}, \pi_{2}\right) \mapsto\left(\mathrm{i} t,-\pi_{1}\right.$, i $\left.\pi_{2}\right)$ of order four of the system (5.52). This analytic continuation converges for $t$ running to infinity in the direction of the positive imaginary axis to the other equilibrium point $\left(\pi_{1}, \pi_{2}\right)=(-1,0)$ of the limit system of $(5.52)$ for $t=\infty$. Applying this analytic continuation a second and third time, one obtains a solution which converges to $\left(\pi_{1}, \pi_{2}\right)=(1,0)$ and $\left(\pi_{1}, \pi_{2}\right)=(-1,0)$ for $t$ running to infinity in the direction of the negative real and imaginary axis, respectively. In this way each of the four types of truncated solutions, for $t$ running to infinity in the direction of the positive and negative real axis with $\left(\pi_{1}(t), \pi_{2}(t)\right)$ converging to $(1,0)$, and for $t$ running to infinity in the direction of the positive and negative imaginary axis with $\left(\pi_{1}(t), \pi_{2}(t)\right)$ converging to $(-1,0)$, are obtained by analytic continuation from the solutions $p_{t_{0}, a^{-}}(t)=p_{l}(t)$ in Lemma 5.2 and 5.6 .

Similarly, the analytic continuation of $p_{\uparrow}(t)$ along the path $t \mathrm{e}^{\mathrm{i} \theta}$ is equal to $p_{\downarrow}(t)$ if $\theta \in \mathbb{R}$ runs from 0 to $5 \pi$. As this agrees with the symmetry $\left(t, \pi_{1}, \pi_{2}\right) \mapsto\left(-t, \pi_{1},-\pi_{2}\right)$ of the system (5.52), it follows that $p_{\downarrow}(t)^{+}=p_{\uparrow}(-t)^{-}$and $p_{\downarrow}(t)^{-}=p_{\uparrow}(-t)^{+}$. If $\theta$ runs from 0 to $5 \pi / 2$ and $15 \pi / 2$, then the analytic continuation of $p_{\uparrow}(t)$ is equal to the two triply truncated solutions near $\left(\pi_{1}, \pi_{2}\right)=(-1,0)$. In this way each of the four triply truncated solutions, for $t$ running to infinity in the upper and lower half plane with $\left(\pi_{1}(t), \pi_{2}(t)\right)$ converging to $(1,0)$ and for $t$ running to infinity in the left and right half plane with $\left(\pi_{1}(t), \pi_{2}(t)\right)$ converging to $(-1,0)$, is obtained by analytic continuation from the solution $p_{\uparrow}(t)$ in Lemma 5.3 , 5.5, and 5.6 .

Because the system 5.52 is real, it has the symmetry $\left(t, \pi_{1}, \pi_{2}\right) \mapsto\left(\bar{t}, \overline{\pi_{1}}, \overline{p_{2}}\right)$. Therefore, if $\left(\pi_{1}(t), \pi_{2}(t)\right)$ is a truncated solution of $(5.52)$ in the sense that it converges to $(1,0)$ as $t$ runs to infinity in the direction of the positive real axis, then $t \mapsto\left(\overline{\pi_{1}(\bar{t})}, \overline{\pi_{2}(\bar{t})}\right)$ is a solution of $(5.52)$ with the same limit behavior, and therefore is truncated in the same way. The triply truncated solutions satisfy $p_{\downarrow}(t)=\overline{p_{\uparrow}(\bar{t})}$, which in combination with $p_{\downarrow}(t)^{ \pm}=p_{\uparrow}(-t)^{\mp}$ implies that $p_{\uparrow}(t)^{ \pm}=\overline{p_{\uparrow}(-\bar{t})^{\mp}}$. The corresponding triply truncated solution of $(5.52)$ satisfies $\pi_{1}(t)=\overline{\pi_{1}(-\bar{t})}$ and $\pi_{2}(t)=-\overline{\pi_{2}(-\bar{t})}$.

Lemma 5.14. The Stokes constant $S$ in (5.34) is nonzero and purely imaginary.

Proof. Because $p_{\downarrow}(t)=\overline{p_{\uparrow}(\bar{t})}$, the asymptotic identity for $t \in \mathbb{R}, t \rightarrow \infty$ implies that $S$ is purely imaginary. If $S=0$, then $p_{\downarrow}(t)=p_{\uparrow}(t)$ in the right half plane, and therefore $p_{\uparrow}(t)$ and $p_{\downarrow}(t)$ would have a common extension to a small solution $p(t)$ for $-(3 / 2) \pi \leq \arg (t) \leq(3 / 2) \pi,|t| \geq r$, when the corresponding solution $y(x)$ would be bounded by a constant times $|x|^{1 / 2}$ for $-(6 / 5) \pi \leq \arg (x) \leq$ $(6 / 5) \pi$ and $|x| \geq R$ for some $R$. As this implies that the single valued function $y(x)$ has no poles for large $|x|$, it would follow that $y(x)$ has only finitely many poles, in contradiction with Corollary 4.7.

Remark 5.15. Because $\tau(t)=\mathrm{e}^{-t} t^{-1 / 2}$, the positive and negative imaginary axis are transitional domains as in the paragraphs preceding Lemma 5.8, the Stokes constant $S$ is equal to the one in Costin [6, (2.8)]. According to [6, Note (3) on p. 7], $S=\mathrm{i} \sqrt{6 / 5 \pi}$. The formula $p_{\downarrow}(t)-p_{\uparrow}(t) \sim$ $\mathrm{i} \sqrt{6 / 5 \pi} \mathrm{e}^{-t} t^{-1 / 2}$ as $-\pi / 2 \leq \arg t \leq \pi / 2,|t| \rightarrow \infty$ agrees, at least up to the sign, with Kapaev [19, Cor. 2.4].

Figure 5.3 shows a region in the $x$-plane, the plane of definition of the solution of (1.1), where the corresponding truncated solution of $(2.2)$ is close to one of the two equilibrium points of the 
limit system 5.1 . This region is the image under the mapping $t \mapsto-2^{-3 / 5} 3^{-1 / 5}((5 / 4) t)^{4 / 5}$ of a domain of the form $R_{\eta, r}$.

The triply truncated solution $p_{\uparrow}(t)$ of $(2.2)$ is defined on a domain determined by the inequalities $|t| \geq r$ and

$$
-\arccos ((-(1 / 2) \log |t|-\log \eta) /|t|) \leq \arg t \leq \pi+\arccos ((-(1 / 2) \log |t|-\log \eta) /|t|) .
$$

Here $r$ and $\eta$ are sufficiently large and small strictly positive real numbers, and arccos denotes the inverse of the bijective function $\cos :[0, \pi] \mapsto[-1,1]$. As the inequalities for $|t|$ and $\arg t$ allow points $t$, $t^{\prime}$ such that $\left|t^{\prime}\right|=|t|$ and $\arg t^{\prime}=\arg t+2 \pi$, the function $p_{\uparrow}(t)$ is interpreted as multi-valued. The properties $p_{\uparrow}(t)^{ \pm}=\overline{p_{\uparrow}(-\bar{t})^{\mp}},(5.34)$, and $p_{\downarrow}(t)=\mathrm{O}\left(t^{-1}\right)$ when $t$ runs to infinity in the direction of the negative imaginary axis imply that for $\arg t=-\pi / 2$ and $\arg t=(3 / 2) \pi$ we have $p_{\uparrow}(t) \sim \mathrm{e}^{-t} t^{-1 / 2}(0,-S)$ and $p_{\uparrow}(t) \sim \mathrm{e}^{t} t^{-1 / 2}(-\mathrm{i} \bar{S}, 0)$ as $|t| \rightarrow \infty$, respectively. As Lemma 5.14 implies that $S \neq 0$, the two branches of $p_{\uparrow}(t)$ do not coincide on the overlap.

The image under the mapping $t \mapsto x=-2^{-3 / 5} 3^{-1 / 5}((5 / 4) t)^{4 / 5}$ of the aforementioned domain where $p_{\uparrow}(t)$ is small is a domain in the $x$-plane where $|x|$ is large and $\arg (x)$ runs from slightly smaller than $(3 / 5) \pi$ to slightly larger than $(11 / 5) \pi$. The other truncated and triply truncated regions are obtained from these by applying a rotation in the $x$-plane over $k 2 \pi / 5,1 \leq k \leq 4$. Because the truncated and triply truncated solutions of $(2.2)$ are bounded in their domains of definition, they have no pole there, and therefore the corresponding truncated and triply truncated solutions of (1.1) have no poles in the aforementioned truncated and and triply truncated regions in the $x$-plane.

Figure 5.4 shows the unique triply truncated region in the $x$-plane which is invariant under complex conjugation. If $y(x)$ denotes the corresponding triply truncated solution of (1.1), the function $x \mapsto \overline{y(\bar{x})}$ is a solution of 1.1 which is triply truncated in the same domain. The uniqueness of triply truncated solutions of (2.2) leads to the following observation of Joshi and Kitaev [14, Cor. 3].

Lemma 5.16. Let $D$ denote the triply truncated domain in Fig. 5.4 which is invariant under complex conjugation. Then the solution $y(x)$ of (1.1) which is triply truncated on $D$ is real in the sense that $y(x)=\overline{y(\bar{x})}$ for every $x \in \mathbb{C}$. In particular $y(x) \in \mathbb{R}$ for every $x \in \mathbb{R}$ not equal to a pole point of $y$.

The next lemma discusses what happens with Lemma 5.11 in the case of the Boutroux-Painlevé system. Our results correspond to [7, (113)] with the formulas for $H_{0}(\xi)$ and $H_{1}(\xi)$ on [7, p. 38], as $\pi_{1}(x)=Y(x)=1-4 / 25 x^{2}+h(x)$, see [7, p. 36], hence $\pi_{1,0}(\xi)=H_{0}(\xi)+1, \pi_{1,2}(\xi)=$ $H_{2}(\xi)-4 / 25$ and $\left.\pi_{1, l}(\xi)=H_{(} \xi\right)$ for all $l \notin\{0,2\}$. O. and R. Costin [7, p. 39] wrote: "We omit the straightforward but quite lengthy inductive proof that all $H_{k}$ are rational functions of $\xi$." And on p. 40: "For large $\xi$ induction shows that $H_{n} \sim$ Const $_{n} \xi^{n}, \ldots$ ", but did not give further details of the proof.

Lemma 5.17. With the notation of Lemma 5.11, the solution $\left(\pi_{1}(t), \pi_{2}(t)\right)$ of 5.52) corresponding to $p_{C}(t)$ has the asymptotic expansion

$$
\pi_{k}(t)=\sum_{l=0}^{m-1} t^{-l} \pi_{k, l}(C \tau(t))+\mathrm{O}\left(t^{-m}\right)
$$

as $t \in V,|t| \rightarrow \infty$. Here $\pi_{1, l}(\xi)=(\xi-12)^{-l-2} P_{1, l}(\xi)$ and $\pi_{2, l}(\xi)=(\xi-12)^{-l-3} P_{2, l}(\xi)$, where $P_{1, l}(\xi)$ and $P_{2, l}(\xi)$ is a polynomial in $\xi:=C \tau$ of degree $\leq 2 l+2$ and $\leq 2 l+3$, respectively. We 
have

$$
\begin{aligned}
& P_{1,0}(\xi)=(\xi-12)^{2}+144 \xi \\
& P_{2,0}(\xi)=144 \xi(\xi+12) \\
& P_{1,1}(\xi)=\xi\left(216+210 \xi+3 \xi^{2}-\xi^{3} / 60\right) \\
& P_{2,1}(\xi)=\left(497664-134784 \xi+266112 \xi^{2}+25704 \xi^{3}-24 \xi^{4}+\xi^{5}\right) / 60
\end{aligned}
$$

Proof. It follows from the last paragraph in Lemma 5.8 that it suffices to prove all the formulas for $C=1$, when $\tau=\xi$.

The shortest proof of the formulas for $\pi_{1,0}(\xi)$ and $\pi_{2,0}(\xi)$ is to verify that these functions satisfy the differential equations $-\xi \mathrm{d} P_{1,0} / \mathrm{d} \xi=P_{2,0},-\xi \mathrm{d} P_{2,0} / \mathrm{d} \xi=\left(P_{1,0}{ }^{2}-1\right) / 2$ corresponding to (5.40), with the initial conditions $P_{1,0}(0)=1, P_{2,0}(0)=0$, and the derivatives with respect to $\xi$ at $\xi=0$ of $p^{+}=\left(P_{1,0}+P_{2,0}-1\right) / 2$ and $p^{-}=\left(P_{1,0}-P_{2,0}-1\right) / 2$ equal to 0 and $C$, respectively. The longer proof below explains how the formulas for $\pi_{1,0}(\xi)$ and $\pi_{2,0}(\xi)$ could have been found.

The system $(5.52)$ is equivalent to the second order differential equation

$$
\frac{\mathrm{d}^{2} \pi}{\mathrm{d} t^{2}}=-\frac{1}{t} \frac{\mathrm{d} \pi}{\mathrm{d} t}+\frac{1}{2}\left(\pi^{2}-1\right)+\frac{4}{25 t^{2}} \pi
$$

for $\pi(t)=\pi_{1}(t)$, when $\pi_{2}(t)$ is given in terms of $\pi(t)$ by means of the formula

$$
\pi_{2}(t)=\frac{\mathrm{d} \pi(t)}{\mathrm{d} t}+\frac{2}{5 t} \pi(t) .
$$

The autonomous limit equation of $(5.60)$ for $t \rightarrow \infty$ is $\Pi^{\prime \prime}=\left(\Pi^{2}-1\right) / 2$, a Newton equation with potential energy $V(\Pi):=-\Pi^{3} / 6+\Pi / 2$. It follows that the total energy $E=\left(\Pi^{\prime}\right)^{2} / 2+V(\Pi)$ is a constant of motion, and the solution which converges to the equilibrium point $\left(\Pi, \Pi^{\prime}\right)=(1,0)$ has energy $E=V(1)=1 / 3$. This leads to the first order differential equation $\left(\Pi^{\prime}\right)^{2}=2(1 / 3-V(\Pi))=$ $(\Pi-1)^{2}(\Pi+2) / 3$, hence, if the independent variable is denoted by $s, \mathrm{~d} s / \mathrm{d} \Pi=3^{1 / 2}(\Pi-1)^{-1}(\Pi+$ $2)^{1 / 2}$, when the substitution $\Pi+2=\Psi^{2}$ leads to

$$
\begin{aligned}
& s= 2 \sqrt{3} \int^{\Psi(s)}\left(\Psi^{2}-3\right)^{-1} \mathrm{~d} \Psi+c=\log \frac{\Psi(s)-\sqrt{3}}{\Psi(s)+\sqrt{3}}+c \\
& \Leftrightarrow \quad \frac{\Psi(s)+\sqrt{3}}{\Psi(s)-\sqrt{3}}=c \mathrm{e}^{-s}=: c \xi \Leftrightarrow \Psi(s)=\sqrt{3} \frac{c \xi+1}{c \xi-1} \\
& \Rightarrow \quad \Pi(s)=\Psi(s)^{2}-2=1+\frac{12 c \xi}{(c \xi-1)^{2}},
\end{aligned}
$$

where $c$ denotes a constant which at every place might be a different one. The function $s \mapsto \Pi(s)$ is the "degenerate elliptic function" of [7, p. 38]. With the substitution $\xi=\mathrm{e}^{-s}$, the differential equation (5.40) is equivalent to $\mathrm{d} F_{0} / \mathrm{d} s=v\left(0, F_{0}\right)$. As the derivative of $F_{0}^{+}(\xi)$ and $F_{0}^{-}(\xi)$ at $\xi=0$ have to be equal to 0 and 1 , respectively, the derivative of $\Pi=\pi_{1}=1+p^{+}+p^{-}$with respect to $\xi$ at $\xi=0$ has to be equal to 1 . Therefore $c=1 / 12$ and $\Pi=1+\xi /(\xi / 12-1)^{2}$, which proves $\pi_{1,0}(\xi)=P_{1,0}(\xi) /(\xi-12)^{2}$ with $P_{1,0}(\xi)$ as in 5.56). The formula 5.61 with $\mathrm{d} / \mathrm{d} t=(-1-1 / 2 t) \xi \mathrm{d} / \mathrm{d} \xi$ yields

$$
\pi_{2,0}=-\xi \mathrm{d} \pi_{1,0} / \mathrm{d} \xi=P_{2,0}(\xi) /(\xi-12)^{3},
$$

with $P_{2,0}(\xi)$ as in (5.57).

Because $\pi_{1,0}$ and $\pi_{2,0}$ are only singular at $\xi=12$, it follows from Lemma 5.8 and Lemma 5.11 that the functions $\pi_{1, l}$ and $\pi_{2, l}$ have a complex analytic continuation along any path in $\mathbb{C} \backslash\{12\}$, and that the asymptotic expansion $(5.55)$ holds along these paths. If $\xi$ runs around 12 along a 
small circle, then the $t_{n}(\xi)$ with large modulus, see Lemma 5.10, return to the same value. As the Painlevé property implies that the function $p_{C}(t)$ is single valued, the asymptotic expansion (5.55) implies by induction on $l$ that $\pi_{1, l}$ and $\pi_{2, l}$ are single valued complex analytic functions on $\mathbb{C} \backslash\{12\}$.

For our system (5.52) the differential equations (5.41) for $i \in \mathbb{Z}_{>0}$ take the form

$$
\begin{aligned}
-\xi \frac{\mathrm{d} \pi_{1, i}}{\mathrm{~d} \xi} & =\pi_{2, i}+\frac{1}{2} \xi \frac{\mathrm{d} \pi_{1, i-1}}{\mathrm{~d} \xi}+\left(i-\frac{7}{5}\right) \pi_{1, i-1} \\
-\xi \frac{\mathrm{d} \pi_{2, i}}{\mathrm{~d} \xi} & =\pi_{1,0} \pi_{1, i}+\frac{1}{2} \xi \frac{\mathrm{d} \pi_{2, i-1}}{\mathrm{~d} \xi}+\left(i-\frac{8}{5}\right) \pi_{2, i-1}+\frac{1}{2} \sum_{j=1}^{i-1} \pi_{1, j} \pi_{1, i-j} .
\end{aligned}
$$

Given $\pi_{1, j}$ and $\pi_{2, j}$ for $j<i$, (5.62), (5.63) is an inhomogeneous linear system of first order differential equations for $\left(\pi_{1, i}, \pi_{2, i}\right)$, equivalent to the inhomogenous linear second order differential equation

$$
\begin{aligned}
& \xi^{2} \frac{\mathrm{d}^{2} \pi_{1, i}}{\mathrm{~d} \xi^{2}}+\xi \frac{\mathrm{d} \pi_{1, i}}{\mathrm{~d} \xi}=-\xi \frac{\mathrm{d}}{\mathrm{d} \xi}\left(-\xi \frac{\mathrm{d} \pi_{1, i}}{\mathrm{~d} \xi}\right) \\
= & \pi_{1,0} \pi_{1, i}+\frac{1}{2} \xi \frac{\mathrm{d} \pi_{2, i-1}}{\mathrm{~d} \xi}+\left(i-\frac{8}{5}\right) \pi_{2, i-1}+\frac{1}{2} \sum_{j=1}^{i-1} \pi_{1, j} \pi_{1, i-j} \\
& -\xi \frac{\mathrm{d}}{\mathrm{d} \xi}\left(\frac{1}{2} \xi \frac{\mathrm{d} \pi_{1, i-1}}{\mathrm{~d} \xi}+\left(i-\frac{7}{5}\right) \pi_{1, i-1}\right)
\end{aligned}
$$

for $\pi_{1, i}$, when $\pi_{2, i}$ can be solved from $\left(5.62\right.$ in terms of $\pi_{1, i}$ and $\pi_{1, i-1}$.

Let $\varphi_{1}(\xi)$ and $\varphi_{2}(\xi)$ be a basis of solutions of the homogeneous linear second order differential equation $\pi^{\prime \prime}=a(\xi) \pi^{\prime}+b(\xi) \pi$. Lagrange's method of variations of constants yields that the solutions of the inhomogeneous equation $\pi^{\prime \prime}=a(\xi) \pi^{\prime}+b(\xi) \pi+f(\xi)$ are of the form

$$
\pi(\xi)=c_{1} \varphi_{1}(\xi)+c_{2} \varphi_{2}(\xi)+\int_{\xi_{0}}^{\xi}\left(-\varphi_{1}(\xi) \varphi_{2}(\eta)+\varphi_{2}(\xi) \varphi_{1}(\eta) \frac{f(\eta)}{w(\eta)} \mathrm{d} \eta\right.
$$

Here $w=\varphi_{1} \varphi_{2}^{\prime}-\varphi_{1}^{\prime} \varphi_{2}$ is the Wronskian determinant, which satisfies $w^{\prime}=a w$, hence

$$
w(\xi)=w\left(\xi_{0}\right) \mathrm{e}^{\int_{\xi_{0}}^{\xi} a(\eta) \mathrm{d} \eta} .
$$

The differential equation $\pi^{\prime \prime}=a(\xi) \pi^{\prime}+b(\xi) \pi$ has a regular singular point at $\xi=\Xi$ if $a(\xi)$ and $b(\xi)$ have a pole of order $\leq 1$ and $\leq 2$ at $\xi=\Xi$. If $a(\xi)=A(\xi-\Xi)^{-1}+\mathrm{O}(1)$ and $b(\xi)=B(\xi-\Xi)^{-2}+$ $\mathrm{O}\left((\xi-\Xi)^{-1}\right)$ as $\xi \rightarrow \Xi$, and the indicial equation $\lambda(\lambda-1)=A \lambda+B$ has to distinct solutions $\lambda_{1}$ and $\lambda_{2}$, then there is a basis of solutions $\varphi_{1}(\xi)$ and $\varphi_{2}(\xi)$ such that $\varphi_{k}(\xi)=(\xi-\Xi)^{\lambda_{k}}(1+\mathrm{o}(1))$, and $w(\xi)=\left(\lambda_{2}-\lambda_{1}\right)(\xi-\Xi)^{\lambda_{1}+\lambda_{2}-1}$. See for instance Coddington and Levinson [4, Chap. 4, Sec. 8]. Therefore, if $b(\xi)=\mathrm{O}(\xi-\Xi)^{b}$, then the integral in (5.64) is of order $(\xi-\Xi)^{b+2}$ as $\xi \rightarrow \Xi$. If $\lambda_{1}=\lambda_{2}$, then one has to replace $\varphi_{2}(\xi)=(\xi-\Xi)^{\lambda_{2}}(1+o(1))$ by $\varphi_{2}(\xi)=\left((\xi-\Xi)^{\lambda_{1}} \log (\xi-\Xi)\right)(1+o(1))$, with a corresponding change in the estimate for the integral in (5.64).

At $\xi=12$ we have $A=0$ and $B=(12)^{-2}(12)^{3}=12$, when the solutions of the indicial equation are $\lambda_{1}=-3$ and $\lambda_{2}=4$. If, for every $0 \leq j \leq i-1, \pi_{1, j}$ and $\pi_{2, j}$ are meromorphic at $\xi=12$ with a pole of order $\leq j+2$ and $\leq j+3$, respectively, then an inspection of the inhomogenous terms in the second order diffrential equation for $\pi_{1, i}$ yields $b=-i-4$, and because $b+2=-i-2 \leq-3$ it follows that $\pi_{1, i}$ has a pole of order $\leq i+2$ at $\xi=12$. For $i=1$ we have $b=-4$, but then $\lambda_{1}=-3$ yields that $\pi_{1,1}$ has a pole of order $\leq 3$. Subsequently 5.62 implies that $\pi_{2, i}$ has a pole of order $\leq i+3$ at $\xi=12$. It follows by induction on $l$ that, at $\xi=12, \pi_{1, l}$ and $\pi_{2, l}$ have a pole of order $\leq l+2$ and $\leq l+3$, respectively.

At $\xi=\infty$ we have $A=-1$ and $B=1$, when the solutions of the indicial equation are $\lambda_{1}=1$ and $\lambda_{2}=-1$. If, for every $0 \leq j \leq i-1, \pi_{1, j}$ and $\pi_{2, j}$ are meromorphic at $\xi=\infty$ with exponents 
$\leq j$, then an inspection of the inhomogenous terms in the second order diffrential equation for $\pi_{1, i}$ yields $b=i-2$, and because $b+2=i \geq 1$ it follows that at $\xi=\infty$ the function $\pi_{1, i}$ has an exponent $\leq i$. Subsequently $\left(5.62\right.$ implies that also $\pi_{2, i}$ has an exponent $\leq i$ at $\xi=\infty$. It follows by induction on $l$ that the functions $\pi_{1, l}$ and $\pi_{2, l}$ have exponents $\leq l$ at $\xi=\infty$, and therefore are rational functions of the form $\pi_{1, l}(\xi)=(\xi-12)^{-l-2} P_{1, l}(\xi)$ and $\pi_{2, l}(\xi)=(\xi-12)^{-l-3} P_{2, l}(\xi)$, where $P_{1, l}$ and $P_{2, l}$ are polynomials of degree $\leq 2 l+2$ and $\leq 2 l+3$, respectively.

The functions $\pi_{1,1}(\xi)=(\xi-12)^{-3} P_{1,1}(\xi)$ and $\pi_{2,1}(\xi)=(\xi-12)^{-4} P_{2,1}(\xi)$, with the respective polynomials $P_{1,1}(\xi)$ and $P_{2,1}(\xi)$ as in (5.58) and (5.59), have been found with the help of a formula manipulation computer program, in the following way. The solutions of the system 5.62, (5.63) for $i=1$ which are complex analytic in a neighborhood of $\xi=0$ are of the form

$$
\begin{aligned}
\pi_{1,1}(\xi)= & \xi\left((720 c-84672)+(60 c+4464) \xi+180 \xi^{2}-\xi^{3}\right) /\left(60(\xi-12)^{3}\right), \\
\pi_{2,1}(\xi)= & \left(497664+(8640 c-1306368) \xi+(2880 c-124416) \xi^{2}+(60 c+17568) \xi^{3}\right. \\
& \left.-24 \xi^{4}+\xi^{5}\right) /\left(60(\xi-12)^{4}\right),
\end{aligned}
$$

where $c$ is free constant. With these functions $\pi_{1,1}$ and $\pi_{2,1}$, an investigation of the explicit solutions $\pi_{1,2}, \pi_{2,2}$ of the system (5.62), (5.63) for $i=2$ yields that there exist solutions which are complex analytic in a neighborhood of $\xi=0$ if and only if $c=678 / 5$. Therefore $\pi_{1,1}(\xi)=(\xi-12)^{-3} P_{1,1}(\xi)$ and $\pi_{2,1}(\xi)=(\xi-12)^{-4} P_{2,1}(\xi)$ with $P_{1,1}(\xi)$ and $P_{2,1}(\xi)$ as in 5.58) and (5.59), respectively.

As the system (5.52) is just a rescaled version of 2.2 , passing to the complex projective plane and successively blowing up the base points of the vector fields, as in Section 2, leads to surface $S_{9}$, with a locus $I$ where the vector field is infinite equal to the union of nine complex projective lines $L_{i}^{(9-i)}, 0 \leq i \leq 8$, and a pole line $L_{9} \backslash I$, where $L_{9}$ is the complex projective line appearing at the last blowup. In the common domain of definition of the coordinate systems $\left(\pi_{i j 1}, \pi_{i j 2}\right)$ and $\left(\pi_{1}, \pi_{2}\right)$, an application of the birational transformation $\left(\pi_{1}, \pi_{2}\right) \mapsto\left(\pi_{i j 1}, \pi_{i j 2}\right)$ to the asymptotic expansion (5.55) leads to an asymptotic expansion

$$
\pi_{i j k}(t)=\sum_{l=0}^{m-1} t^{-l} \pi_{i j k, l}(\tau(t))+\mathrm{O}\left(t^{-m}\right)
$$

as $t \in V,|t| \rightarrow \infty$, where the functions $\pi_{i j k, l}(\tau)$ are rational expressions in the functions $\pi_{1, l^{\prime}}(\tau)$, $\pi_{2, l^{\prime}}(\tau)$ for $0 \leq l^{\prime} \leq l$, and therefore are rational functions of $\tau$. Because the differential equations for $\pi_{i j k, 0}(\tau)$, analogous to 5.40 , correspond to the autonomous limit system, these differential equations are regular and its solutions have a complex analytic extension as long as they remain in in the complement of the inifinity set $I$ in the coordinate system $\left(\pi_{i j 1}, \pi_{i j 2}\right)$. Because also the nonautonomous vector field is regular in $S \backslash I$, the functions $\pi_{i j k, l}(\tau)$ with $l \in \mathbb{Z}_{>0}$ satisfy inhomogeneous linear differential equations as (5.41), variational equations of the differential equations for $\pi_{i j k, 0}(\tau)$, where the inhomogeneous term is a regular expression in the $\pi_{i j k, m}(\tau)$ with $m<l$. It follows by induction on $l$ that all the rational functions $\pi_{i j k, l}(\tau)$ are regular, when the perturbation argument in the last paragraph of the proof of Lemma 5.11 yields that the asymptotic expansion (5.65) extends to the whole complement of $I$ in the coordinate system $\left(\pi_{i j 1}, \pi_{i j 2}\right)$.

The pole line is visible in the coordinate system $\left(\pi_{911}, \pi_{912}\right)$ as the line $\pi_{912}=0$, and therefore the poles are the solutions $T$ of the equation $\pi_{912}(T)=0$, where $\pi_{912}=\pi_{1} / \pi_{2}$. This leads to the following asymptotic results for the poles, where in view of (5.54) the poles of the corresponding truncated solution of 1.1 are given by $X_{n}=-2^{-3 / 5} 3^{-1 / 5}\left(5 T_{n} / 4\right)^{4 / 5}$.

Lemma 5.18. There exist universal sequences of coefficients $c_{j}, d_{k, l} j, k, l \in \mathbb{Z}_{\geq 0}$, with $c_{0}=12$, $c_{1}=109 / 10$, and $d_{0,0}=0$, such that following holds. Let $C \neq 0$, and let $p_{C}(t)$ be the solution in Lemma 5.11, of the system (5.3) obtained from (5.52) by means of the substitutions $\pi_{1}=1+p^{+}+p^{-}$, 
$\pi_{2}=p^{+}-p^{-}$. Let $\left(\pi_{1}(t), \pi_{2}(t)\right)$ be the corresponding solution of (5.52). Then there is a sequence of poles $T_{n}, n \in \mathbb{Z}, n>>0$ of $\pi_{1}(t)$, such that $T_{n}=2 \pi \mathrm{i} n(1+\mathrm{o}(1))$ as $n \rightarrow \infty$, and

$$
\tau\left(T_{n}\right):=\mathrm{e}^{-T_{n}} T_{n}{ }^{-1 / 2} \sim \frac{1}{C} \sum_{j=0}^{\infty} c_{j} T_{n}^{-j} \text { as } n \rightarrow \infty .
$$

Furthermore, with the notations

$$
u:=\frac{1}{2 \pi \mathrm{i} n}, \quad v:=\frac{\log (2 \pi \mathrm{i} n)}{2 \pi \mathrm{i} n}, \quad \text { and } \quad W:=u \log \frac{C}{12}-\frac{v}{2},
$$

where $\log (2 \pi \mathrm{i} n)=\log (2 \pi n)+\pi \mathrm{i} / 2$, we have the more explicit but more complicated asymptotic expansion

$$
T_{n} \sim 2 \pi \mathrm{i} n-\frac{1}{2} \log (2 \pi \mathrm{i} n)+\log \frac{C}{12}+\sum_{k, l \geq 0} d_{k, l} u^{k} W^{l} \quad \text { as } \quad n \rightarrow \infty,
$$

of which the leading terms yield

$$
\begin{aligned}
T_{n}= & 2 \pi \mathrm{i} n-\frac{1}{2} \log (2 \pi \mathrm{i} n)+\log \frac{C}{12}+\frac{v}{4}-\left(\frac{1}{2} \log \frac{C}{12}+\frac{109}{120}\right) u \\
& +\frac{1}{16} v^{2}-\left(\frac{1}{4} \log \frac{C}{12}+\frac{139}{240}\right) u v+\mathrm{O}\left(n^{-2}\right)
\end{aligned}
$$

as $n \rightarrow \infty$. If $C \in \mathbb{C} \backslash\{0\}$ runs once around the origin in the positive direction, then $T_{n}$ moves continuously to $T_{n+1}$.

Conversely, for every $\eta>0$ there exists an $r>0$, such that these $T_{n}$ are the only poles $T$ of $\pi_{1}(t)$ such that $|T| \geq r, \operatorname{Im} T \geq 0$, and $\left|\mathrm{e}^{-T} T^{-1 / 2}\right| \leq \eta$.

Proof. It follows from Lemma 5.17 that the poles $T$ of $\pi_{1}(t)$ with bounded $\Xi(T):=C \mathrm{e}^{-T} T^{-1 / 2}$ satisfy $\Xi(T) \rightarrow 12$ as $|T| \rightarrow \infty$.

There exists a sequence of rational functions $\pi_{912, l}, l \in \mathbb{Z}_{\geq 0}$, such that, with the notation $\xi=C \tau=C \mathrm{e}^{-t} t^{-1 / 2}$,

$$
\pi_{912}(t)=\frac{\pi_{1}(t)}{\pi_{2}(t)}=\sum_{l=0}^{m-1} t^{-l} \pi_{912, l}(\xi)+\mathrm{O}\left(t^{m}\right),
$$

for every $m \in \mathbb{Z}_{>0}$. Lemma 5.17 implies that

$$
\begin{aligned}
\pi_{912,0}(\xi)= & (\xi-12)\left(144+120 \xi+\xi^{2}\right) /(144 \xi(\xi+12)) \text { and } \\
\pi_{912,1}(\xi)= & \left(-71663616-40310784 \xi-248832 \xi^{2}-11860992 \xi^{3}-1221696 \xi^{4}\right. \\
& \left.+1224 \xi^{5}-240 \xi^{6}-\xi^{7}\right) /\left(\left(1244160 \xi^{2}(\xi+12)^{2}\right) .\right.
\end{aligned}
$$

Because $\pi_{912,0}(12)=0$ and $\pi_{912,0}^{\prime}(12)=1 / 24 \neq 0$, an application of the implicit function theorem yields that there exist open neighbourhoods $A$ and $B$ of 12 and 0 , respectively, such that for every $t^{-1} \in A$ the equation

$$
\sum_{l=0}^{m-1} t^{-l} \pi_{912, l}(\xi)=0
$$

has a unique solution $\Xi_{m}=\Xi_{m}\left(t^{-1}\right) \in B$, which moreover depends in a complex analytic fashion on $t^{-1}$, and satisfies $\Xi_{m}(0)=12$. Furthermore, $\Xi(T)=\Xi_{m}\left(T^{-1}\right)+\mathrm{O}\left(T^{-m}\right)$, and as the left hand side does not depend on $m$, it follows that the coefficients $c_{j}$ for $0 \leq j \leq m-1$ in the Taylor expansion of the function $\Xi_{m}$ at the origin do not depend on $m$. Because this holds for every $m \in \mathbb{Z}_{>0}$, it 
follows that there is a sequence of complex numbers $c_{j}, j \in \mathbb{Z}_{>0}$, such that the poles $T$ of $\pi_{1}(t)$ with bounded $\Xi(T):=C \mathrm{e}^{-T} T^{-1 / 2}$ satisfy

$$
C \tau(T)=C \mathrm{e}^{-T} T^{-1 / 2} \sim 12+\sum_{j>0} c_{j} T^{-j} \quad \text { as } \quad|T| \rightarrow \infty .
$$

Because $\pi_{912,1}(12)=-109 / 240$, we have $c_{1}=-\pi_{912,1}(12) / \pi_{912,0}^{\prime}(12)=109 / 10$. This completes the proof of (5.66).

The proof of (5.67) is analogous to the proof of (5.46). For any $m \in \mathbb{Z}_{>0},(5.66)$ yields that

$$
\tau=\tau\left(T_{n}\right)=\frac{12}{C}\left(1+\frac{1}{12} \sum_{j=1}^{m-1} c_{j} T_{n}^{-j}+r\right),
$$

where $r=\mathrm{O}\left(T_{n}{ }^{-m}\right)=\mathrm{O}\left(n^{-m}\right)$, hence

$$
\log \tau=\log \frac{12}{C}+\log \left(1+\sum_{j=1}^{m-1} \frac{c_{j}}{12} T_{n}^{-j}+r\right)
$$

Upon the substitution

$$
\begin{aligned}
T_{n} & =2 \pi \mathrm{i} n-\frac{1}{2} \log (2 \pi \mathrm{i} n)+\log \frac{C}{12}+S \\
& =2 \pi \mathrm{i} n\left(1-\frac{1}{2} v+u \log (C / 12)+u S\right)=2 \pi \mathrm{i} n(1+W+u S),
\end{aligned}
$$

which implies

$$
\log T_{n}=\log (2 \pi \mathrm{i} n)+\log (1+W+u S)
$$

and

$$
T_{n}{ }^{-1}=u(1+W+u S)^{-1}
$$

the equation $T_{n}=2 \pi \mathrm{i} n-(1 / 2) \log T_{n}-\log \tau$ is equivalent to the equation

$$
S=F(u, W, r, S):=-\frac{1}{2} \log (1+W+u S)-\log \left(1+\sum_{j=0}^{m-1} \frac{c_{j}}{12} u^{j}(1+W+u S)^{-j}+r\right) .
$$

Because $F(0,0,0, S) \equiv 0$, it follows from the implicit function theorem in the complex analytic setting that there exist open neighborhoods $U, \mathcal{W}, R$, and $\mathcal{S}$ of the origin in $\mathbb{C}$ such that for every $(u, W, r) \in U \times \mathcal{W} \times R$ the equation has a unique solution $S=S_{m}(u, W, r) \in \mathcal{S}$, and that $S_{m}$ is a complex analytic function on $U \times \mathcal{W} \times R$. In our setting

$$
S_{m}(u, W, r)=S_{m}(u, W, 0)+\mathrm{O}(r)=\sum_{k=0}^{m-1} \sum_{l=0}^{m-k} d_{k, l} u^{k} W^{l}+\mathrm{O}\left(n^{-m}\right) .
$$

Here the coefficients $d_{k, l}$ do not depend on $m$ because $S$ does not depend on $m$. This completes the proof of (5.67).

The equation for $m=2$ yields

$$
S=-\frac{1}{2}(W+u S)+\frac{1}{4}\left(W^{2}+2 W u S\right)-\frac{c_{1}}{12} u(1-W)+\mathrm{O}\left(n^{-2}\right),
$$

hence

$$
S=-\frac{1}{2} W-\frac{c_{1}}{12} u+\frac{1}{4} W^{2}+\left(\frac{1}{4}+\frac{c_{1}}{12}\right) W u+\mathrm{O}\left(n^{-2}\right)
$$

which implies (5.68).

If $C \in \mathbb{C} \backslash\{0\}$ runs once around the origin in the positive direction, then (5.66) implies that $\tau\left(T_{n}\right)$ runs once around the origin in the negative direction, when Lemma 5.10 implies that $T_{n}$ moves continuously to $T_{n+1}$. 
Figure 5.5 illustrates the asymptotic approximations of the poles in $(5.68)$ in the complex $t$-plane, of Boutroux's triply truncated solution $p_{\downarrow}(t)$, when, according to Remark 5.15, $C=\mathrm{i} \sqrt{6 / 5 \pi}$. Shown are the points in the right hand side of (5.68) without the remainder term $\mathrm{O}\left(n^{-2}\right)$, for $1 \leq n \leq 20$. For clarity of the picture, the imaginary part has been multiplied by $1 / 24$ in comparison to the real part. It would be interesting to compare the approximate poles in Figure 5.5 with the numerical approximations of the actual poles of $p_{\downarrow}(t)$.

Remark 5.19. The intriguing "General comments 2" of [7, p. 40] say:

"The pole structure can be explored beyond the first array, in much of the same way: For large $\xi$ induction shows that $H_{n} \sim$ Const $\xi^{n}$, suggesting a reexpansion for large $\xi$ in the form

$$
h \sim \sum_{k=0}^{\infty} \frac{H_{k}^{[1]}\left(\xi_{2}\right)}{x^{k}} ; \quad \xi_{2}=C^{[1]} \xi x^{-1}=C C^{[1]} x^{-3 / 2} \mathrm{e}^{-x} .
$$

By the same technique it can be shown that (118) holds and, by matching with

$$
h \sim \sum_{k=0}^{\infty} x^{-k} H_{k}(\xi(x))
$$

at $\xi_{2} \sim x^{-2 / 3}$, we get $H_{0}^{[1]}=H_{0}$ with $C^{[1]}=-1 / 60$. Hence, if $x_{s}$ belongs to the first line of poles, i.e.

$$
\xi\left(x_{s}\right)=\xi_{s}=12+\frac{109}{10 x}+\mathrm{O}\left(x^{-2}\right)
$$

the second line of poles is given by the condition

$$
x_{1}^{-3 / 2} \mathrm{e}^{-x_{1}}=-60 \cdot 12 c
$$

i.e., it is situated at a logarithmic distance from the first one:

$$
x_{1}-x_{s}=-\ln x_{s}+(2 n+1) \pi \mathrm{i}-\ln (60)+\mathrm{o}(1) .
$$

Similarly one finds $x_{s, 3}$ and in general $x_{s, n}$. The second scale for the $n$-array is $x^{-n-1 / 2} \mathrm{e}^{-x}$.

The expansion (113) can however matched directly to an adiabatic invariant-like expansion valid throughout the sector where $h$ has poles, similar to the one in Joshi and Kruskal [16]. In this language, the successive expansions of the form (118) pertain to the separatrix crossing region. We will not pursue this issue here."

The word "suggesting" preceding (118) indicates that (118) is a conjecture, but in the sequel all the statements, including (118), are treated as facts, with only some hints of proofs. The phrase "matching with (113) at $\xi_{2} \sim x^{-2 / 3 "}$ suggests that $H_{n} \sim$ Const $_{n} \xi^{n}$ implies that the expansion (113) extends to domains where $\xi(x)$ is of order $x^{1 / 3}$, thus allowing a matching with (118) for $\xi_{2}(x)=C^{[1]} \xi(x) x^{-1}$ of order $x^{-2 / 3}$.

Write $\tau^{[N]}(t):=\tau(t) t^{-N}=\mathrm{e}^{-t} t^{-N-1 / 2}$, the second scale for the $(N+1)$-st array of poles. Because $\pi_{k, l}(\xi) \sim$ Const $_{k, l} \xi^{l}$ for $\xi \rightarrow \infty$, see Lemma 5.17, the $t^{-l} \pi_{k, l}\left(C \tau(t), l \in \mathbb{Z}_{\geq 0}\right.$ form an asymptotic sequence for $|t| \rightarrow \infty,|\tau(t)|=\mathrm{o}(|t|)$ and $\tau(t)$ bounded away from $12 / C$, in the sense that $t^{-l} \pi_{k, l}\left(C \tau(t)=\mathrm{O}\left(\tau^{[1]}(t)^{l}\right)\right.$, where $\tau^{[1]}(t) \rightarrow 0$. A stronger conjecture would be that $(5.55)$ extends as an asymptotic expansion for $|t| \rightarrow \infty$ in the aforementioned domain. Because (5.56) and (5.57) imply that $\left(\pi_{1,0}(\xi), \pi_{2,0}(\xi)\right) \rightarrow(1,0)$ as $\xi \rightarrow \infty$, it would follow that $\left(\pi_{1}(t), \pi_{2}(t)\right)$ converges to the equilibrium point $(1,0)$ of the autonomous limit system if $|t| \rightarrow \infty,|\tau(t)| \rightarrow \infty$, and $|\tau(t)|=\mathrm{o}(|t|)$. In view of (5.56), (5.57), (5.58), and (5.59), the first two terms of the extended asymptotic expansion yield

$$
\begin{gathered}
\pi_{1}(t)=1+144(C \tau(t))^{-1}-C \tau^{[1]}(t) / 60+\mathrm{o}\left(\tau(t)^{-1}\right)+\mathrm{o}\left(\tau^{[1]}(t)\right), \\
\pi_{2}(t)=144(C \tau(t))^{-1}+C \tau^{[1]}(t) / 60+\mathrm{o}\left(\tau(t)^{-1}\right)+\mathrm{o}\left(\tau^{[1]}(t)\right) . \\
45
\end{gathered}
$$


Therefore, if we restrict to $\tau(t)^{-1}=\mathrm{o}\left(\tau^{[1]}(t)\right)=\mathrm{o}(\tau(t) / t)$, that is $|\tau(t)| /|t|^{1 / 2} \rightarrow \infty$, then

$$
\left(\pi_{1}(t), \pi_{2}(t)\right)=(1,0)+\xi^{[1]}(1,-1)+\mathrm{o}\left(\left(\xi^{[1]}\right)\right) \quad \text { if } \quad \xi^{[1]}(t)=-C \tau^{[1]}(t) / 60
$$

the leading term of an asymptotic expansion

$$
\left(\pi_{1}(t), \pi_{2}(t) \sim \sum_{l \geq 0} t^{-l}\left(\pi_{1, l}^{[1]}\left(\xi^{[1]}(t)\right), \pi_{2, l}^{[1]}\left(\xi^{[1]}(t)\right)\right) .\right.
$$

As in Lemma 5.8 , the function $\xi^{[1]} \mapsto\left(\pi_{1,0}^{[1]}\left(\xi^{[1]}\right), \pi_{2,0}^{[1]}\left(\xi^{[1]}\right)\right)$ satisfies the same differential equation (5.40) as the function $\xi \mapsto\left(\pi_{1,0}(\xi), \pi_{2,0}(\xi)\right)$, where both functions have the same value and first order derivative at the origin. Therefore $\pi_{k, 0}^{[1]}=\pi_{k, 0}$, with $\pi_{1,0}$ and $\pi_{2,0}$ as in Lemma 5.8. It follows, if the aforementioned statements about the asymptotic expansions hold, that the second sequence of poles occurs at points $2 \pi$ i $n-(3 / 2) \log (2 \pi$ i $n)-\log (-720 / C)+\mathrm{o}(1)$, equal to the first sequence $2 \pi$ i $n-(1 / 2) \log (2 \pi$ i $n)-\log (12 / C)+\mathrm{o}(1)$ plus $-\log (2 \pi$ i $n)-\log (-60)+\mathrm{o}(1)$ as $n \rightarrow \infty$.

The text in "General comments 2" of [7, p. 40] continues with the statement that for each $N$ there is an asymptotic expansion of the form

$$
\left(\pi_{1}(t), \pi_{2}(t)\right) \sim \sum_{l=0}^{\infty} t^{-l}\left(\pi_{1, l}^{[N]}\left(C^{[N]} \tau^{[N]}(t)\right), \pi_{2, l}^{[N]}\left(C^{[N]} \tau^{[N]}(t)\right)\right),
$$

valid, if interpreted in the strong sense, for $|t| \rightarrow \infty,\left|\tau^{[N]}(t)\right|=\mathrm{o}(t)$, and $\left|\tau^{[N-1]}(t)\right| \rightarrow \infty$, where the constant $C^{[N]}$ depends linearly on $C$. This would lead to an asymptotic description of the $(N+1)$-st sequence of poles, equal to the $N$-th sequence plus $-\log n+\gamma_{N}+\mathrm{o}(1)$ as $n \rightarrow \infty$, where the constant $\gamma_{N}$ neither depends on $n$ nor on $C$. For $|t| \rightarrow \infty$ and $t$ between the $N$ th and the $(N+1)$-st sequence of poles, the solution $\left(\pi_{1}(t), \pi_{2}(t)\right)$ converges to the equilibrium point $(1,0)$ of the autonomous system, if and only the distance from $t$ to both sequences of poles tends to infinity. Furthermore, for every $M>0$ we have that the energy $E=\pi_{2}^{2} / 2-\pi_{1}^{3} / 6+\pi_{1} / 2$ converges to the critical level $1 / 3$, meaning that the solution converges to the solution of the autonomous limit system at the critical energy level, if $\operatorname{Im} t \rightarrow \infty, \operatorname{Re} t \geq-M \log (\operatorname{Im} t)$, and $|t|$ times the distance from $t$ to the poles tends to infinity. The latter condition is related to the description of the energy near the poles in 3.3 .

We would like to prove statements like those in the second paragraph in the "General comments 2" of [7, p. 40] by means of the averaging method. This is not a trivial matter, as all the asymptotic expansions up till now are near one of the critical values of the energy function, where solutions of the averaged differential equation for the energy function are not uniquely determined. One might expect that the energy function acquires different limit values from the critical value $1 / 3$ of the energy function, if $t$ runs to infinity in the direction of $\mathrm{e}^{\mathrm{i} \theta}$ with $\pi / 2<\theta<3 \pi / 2$. On the other hand the truncated solution converges to the equilibrium point of the autonomous limit system (with energy equal to the critical value $1 / 3$ ) if $-\pi / 2<\theta<\pi / 2$.

\section{Appendix A. OKamoto's Space}

We construct Okamoto's space of initial conditions [21] in the Boutroux rescaling. (See also [10] for the original Painlevé equation (1.1).) Recall that the notation $\left(u_{i j 1}, u_{i j 2}\right)$ will be used to denote the coordinates in the $j$-th chart of the $i$-th blowup and that in each coordinate chart, the Jacobian of the coordinate change from $\left(u_{1}, u_{2}\right)$ to $\left(u_{i j 1}, u_{i j 2}\right)$ will be denoted by

$$
w_{i j}=\frac{\partial u_{i j 1}}{\partial u_{1}} \frac{\partial u_{i j 2}}{\partial u_{2}}-\frac{\partial u_{i j 1}}{\partial u_{2}} \frac{\partial u_{i j 2}}{\partial u_{1}}
$$


Up to and including the seventh blowup, the function $z \dot{E}$ is rational with $w_{i j}$ in the denominator, and we have added the formula for $\dot{E} w_{i j}$ in each coordinate chart.

A.1. Embedding into $\mathbb{P}^{2}$. Recall the embedding of $\left(u_{1}, u_{2}\right)$ into projective space given in Section 1.2. We have the second affine chart in $\mathbb{P}^{2}$ :

$$
\begin{aligned}
u_{021} & =u_{1}^{-1} \\
u_{022} & =u_{1}^{-1} u_{2}, \\
u_{1} & =u_{021}{ }^{-1} \\
u_{2} & =u_{021}{ }^{-1} u_{022}, \\
\dot{u}_{021} & =u_{021}\left(-u_{022}+2(5 z)^{-1}\right), \\
\dot{u}_{022} & =u_{021}{ }^{-1}\left(6+u_{021}^{2}-u_{021} u_{022}^{2}-(5 z)^{-1} u_{021} u_{022}\right), \\
w_{02} & =-u_{021^{3}}, \\
\dot{w}_{02} & =3 u_{021}{ }^{3}\left(u_{022}-2(5 z)^{-1}\right), \\
E w_{02} & =2+u_{021}{ }^{2}-2^{-1} u_{021} u_{022}{ }^{2}, \\
\dot{E} w_{02} & =-(5 z)^{-1}\left(12+2 u_{021}{ }^{2}-3 u_{021} u_{022}{ }^{2}\right) .
\end{aligned}
$$

The line at infinity $L_{0}$ corresponds to $u_{021}=0$. In this chart there are no base points for the Painlevé vector field or the anticanonical pencil.

Third affine chart in $\mathbb{P}^{2}$ :

$$
\begin{aligned}
& u_{031}=u_{2}^{-1} \text {, } \\
& u_{032}=u_{1} u_{2}^{-1} \text {, } \\
& u_{1}=u_{031}^{-1} u_{032}, \\
& u_{2}=u_{031}^{-1} \text {, } \\
& \dot{u}_{031}=-u_{031}{ }^{2}-6 u_{032}{ }^{2}+3(5 z)^{-1} u_{031}, \\
& \dot{u}_{032}=u_{031}{ }^{-1}\left(u_{031}-u_{031}{ }^{2} u_{032}-6 u_{032}{ }^{3}+(5 z)^{-1} u_{031} u_{032}\right) \text {, } \\
& w_{03}=u_{031}{ }^{3} \text {, } \\
& {\left[w_{03} u_{032}{ }^{-3}\right]^{\bullet}=3 u_{031}{ }^{3}\left(-1+2(5 z)^{-1} u_{032}\right) u_{032}{ }^{-4} \text {, }} \\
& E w_{03}=2^{-1} u_{031}-u_{031}{ }^{2} u_{032}-2 u_{032}{ }^{3} \text {, } \\
& \dot{E} w_{03}=(5 z)^{-1}\left(-3 u_{031}+2 u_{031}{ }^{2} u_{032}+12 u_{032}{ }^{3}\right) \text {. }
\end{aligned}
$$

The line at infinity $L_{0}$ corresponds to $u_{031}=0$. Both the The Painlevé vector field and the anticanonical pencil both have a base point $b_{0}$ given by $u_{031}=0, u_{032}=0$.

A.2. Resolution of the flow at $b_{0}$. Blowing up $\mathbb{P}^{2}$ at $b_{0}$ leads to $S_{1}$. 
First coordinate chart:

$$
\begin{aligned}
& u_{031}=u_{111} u_{032}, \\
& u_{032}=u_{112} \text {, } \\
& u_{111}=u_{1}^{-1} \text {, } \\
& u_{112}=u_{1} u_{2}^{-1} \\
& u_{1}=u_{111}{ }^{-1} \text {, } \\
& u_{2}=u_{111}^{-1} u_{112}{ }^{-1} \text {, } \\
& \dot{u}_{111}=u_{112}{ }^{-1} u_{111}\left(-1+2(5 z)^{-1} u_{112}\right) \text {, } \\
& \dot{u}_{112}=u_{111}{ }^{-1}\left(u_{111}-6 u_{112}{ }^{2}-u_{111}{ }^{2} u_{112}{ }^{2}+(5 z)^{-1} u_{111} u_{112}\right) \text {, } \\
& w_{11}=u_{111}^{3} u_{112}{ }^{2}, \\
& {\left[w_{11} u_{112}{ }^{-2}\right]^{\bullet}=3 u_{111^{3}}\left(-1+2(5 z)^{-1} u_{112}\right) u_{112}{ }^{-1} \text {, }} \\
& {\left[w_{11} u_{111}{ }^{-1}\right]^{\bullet}=2 u_{111} u_{112}{ }^{2}\left(-6 u_{112}-u_{111}{ }^{2} u_{112}+3(5 z)^{-1} u_{111}\right) \text {, }} \\
& E w_{11}=2^{-1} u_{111}-2 u_{112}{ }^{2}-u_{111}{ }^{2} u_{112}{ }^{2} \text {, } \\
& \dot{E} w_{11}=(5 z)^{-1}\left(-3 u_{111}+12 u_{112}{ }^{2}+2 u_{111}{ }^{2} u_{112}{ }^{2}\right) \text {. }
\end{aligned}
$$

Then $u_{112}=0$ defines $L_{1}$ and $u_{111}=0$ defines $L_{0}^{(1)}$. The Painlevé vector field and the anticanonical pencil both have a base point $b_{1}$ given by $u_{111}=0, u_{112}=0$.

The second coordinate chart after the first blowup is defined by

$$
\begin{aligned}
u_{031} & =u_{121} \\
u_{032} & =u_{122} u_{031} \\
u_{121} & =u_{2}^{-1}=u_{111} u_{112} \\
u_{122} & =u_{1}=u_{111}^{-1} \\
u_{1} & =u_{122} \\
u_{2} & =u_{121}{ }^{-1} \\
\dot{u}_{121} & =u_{121}\left(-u_{121}-6 u_{121} u_{122}^{2}+3(5 z)^{-1}\right) \\
\dot{u}_{122} & =u_{121}{ }^{-1}\left(1-2(5 z)^{-1} u_{121} u_{122}\right) \\
w_{12} & =u_{121}^{2} \\
\dot{w}_{12} & =2_{121}^{2}\left(-u_{121}-6 u_{121} u_{122}^{2}+3(5 z)^{-1}\right) \\
E w_{12} & =2^{-1}-u_{121}^{2} u_{122}-2 u_{121}^{2} u_{122}{ }^{3} \\
\dot{E} w_{12} & =(5 z)^{-1}\left(-3+2 u_{121}{ }^{2} u_{122}+12 u_{121}^{2} u_{122}{ }^{3}\right)
\end{aligned}
$$

The equation $u_{121}=0$ defines $L_{1}$. The line $L_{0}^{(1)}$ is not visible, and there are no base points in this chart. 
A.3. Resolution of the flow at $b_{1}$. Blowing up $S_{1}$ at $b_{1}$ leads to $S_{2}$. First coordinate chart:

$$
\begin{aligned}
& u_{111}=u_{211} u_{112}, \\
& u_{112}=u_{212} \text {, } \\
& u_{211}=u_{1}^{-2} u_{2} \\
& u_{212}=u_{2}^{-1} u_{1} \\
& u_{1}=u_{211}^{-1} u_{212}{ }^{-1} \text {, } \\
& u_{2}=u_{211}^{-1} u_{212}{ }^{-2} \text {, } \\
& \dot{u}_{211}=u_{212}{ }^{-1}\left(-2 u_{211}+6 u_{212}+u_{211}{ }^{2} u_{212}{ }^{3}+(5 z)^{-1} u_{211} u_{212}\right) \text {, } \\
& \dot{u}_{212}=u_{211}{ }^{-1}\left(u_{211}-6 u_{212}-u_{211}{ }^{2} u_{212}{ }^{3}+(5 z)^{-1} u_{211} u_{212}\right) \text {, } \\
& w_{21}=u_{211^{3}} u_{212}{ }^{4} \text {, } \\
& {\left[w_{21} u_{212}{ }^{-1}\right]^{\bullet}=3 u_{211^{3}} u_{212}{ }^{2}\left(-1+2(5 z)^{-1} u_{212}\right) \text {, }} \\
& {\left[w_{21} u_{211}{ }^{-1}\right]^{\bullet}=2 u_{211} u_{212}^{4}\left(-6-u_{211}{ }^{2} u_{212}{ }^{2}+3(5 z)^{-1} u_{211}\right) \text {, }} \\
& E w_{21}=2^{-1} u_{211}-2 u_{212}-u_{211}{ }^{2} u_{212}{ }^{3} \text {, } \\
& \dot{E} w_{21}=(5 z)^{-1}\left(-3 u_{211}+12 u_{212}+2 u_{211}{ }^{2} u_{212}{ }^{3}\right) \text {. }
\end{aligned}
$$

Then $u_{212}=0$ defines $L_{2}$ and $u_{211}=0$ defines the proper transform $L_{0}^{(2)}$ of $L_{0}^{(1)}$. The proper transform $L_{1}^{(1)}$ of $L_{1}$ is not visible in this chart. The Painlevé vector field and the anticanonical pencil both have a base point $b_{2}$ given by $u_{211}=0, u_{212}=0$.

The second coordinate chart after the second blowup is defined by

$$
\begin{aligned}
u_{111} & =u_{221}, \\
u_{112} & =u_{222} u_{111}, \\
u_{221} & =u_{1}{ }^{-1}=u_{211} u_{212}, \\
u_{222} & =u_{1}^{2} u_{2}^{-1}=u_{211}{ }^{-1}, \\
u_{1} & =u_{221}{ }^{-1} \\
u_{2} & =u_{221}{ }^{-2} u_{222}{ }^{-1} \\
\dot{u}_{221} & =u_{222}{ }^{-1}\left(-1+2(5 z)^{-1} u_{221} u_{222}\right), \\
\dot{u}_{222} & =u_{221}{ }^{-1}\left(2-6 u_{221} u_{222}{ }^{2}-u_{221}{ }^{3} u_{222}{ }^{2}-(5 z)^{-1} u_{221} u_{222}\right), \\
w_{22} & =u_{221}{ }^{4} u_{222}{ }^{2}, \\
\dot{w}_{22} & =2 u_{221}{ }^{2} u_{222}{ }^{2}\left(-6 u_{222}-u_{221}{ }^{2} u_{222}+3(5 z)^{-1}\right), \\
E w_{22} & =2^{-1}-2 u_{221} u_{222}{ }^{2}-u_{221}{ }^{3} u_{222}{ }^{2}, \\
\dot{E} w_{22} & =(5 z)^{-1}\left(-3+12 u_{221} u_{222}{ }^{2}+2 u_{221}{ }^{3} u_{222}{ }^{2}\right) .
\end{aligned}
$$

The equations $u_{221}=0$ and $u_{222}=0$ define $L_{2}$ and $L_{1}^{(1)}$, respectively. The line $L_{0}^{(2)}$ is not visible, and there are no base points in this chart. 
A.4. Resolution of the flow at $b_{2}$. Blowing up $S_{2}$ at $b_{2}$ leads to $S_{3}$. First coordinate chart:

$$
\begin{aligned}
& u_{211}=u_{311} u_{212}, \\
& u_{212}=u_{312} \text {, } \\
& u_{311}=u_{1}^{-3} u_{2}^{2} \text {, } \\
& u_{312}=u_{1} u_{2}{ }^{-1} \text {, } \\
& u_{1}=u_{311}^{-1} u_{312}{ }^{-2} \text {, } \\
& u_{2}=u_{311}^{-1} u_{312}{ }^{-3} \text {, } \\
& \dot{u}_{311}=u_{312}{ }^{-1}\left(12-3 u_{311}+2 u_{311^{2}} u_{312}{ }^{4}\right) \text {, } \\
& \dot{u}_{312}=u_{311}^{-1}\left(-6+u_{311}-u_{311}^{2} u_{312}^{4}+(5 z)^{-1} u_{311} u_{312}\right), \\
& w_{31}=u_{311}{ }^{3} u_{312}{ }^{6} \text {, } \\
& {\left[w_{31}\left(u_{311}-4\right)^{-1}\right]^{\bullet}=2 u_{311}{ }^{3} u_{312}{ }^{6}\left(u_{311}-4\right)^{-2}\left(-u_{311}{ }^{2} u_{312}{ }^{3}+3(5 z)^{-1}\left(u_{311}-4\right)\right) \text {, }} \\
& E w_{31}=-2+2^{-1} u_{311}-u_{311}{ }^{2} u_{312}{ }^{4}, \\
& \dot{E} w_{31}=(5 z)^{-1}\left(12-3 u_{311}+2 u_{311}{ }^{2} u_{312}{ }^{4}\right) \text {. }
\end{aligned}
$$

Then $u_{312}=0$ defines $L_{3}$ and $u_{311}=0$ defines the proper transform $L_{0}^{(3)}$ of $L_{0}^{(2)}$. The proper transforms $L_{2}^{(1)}$ of $L_{2}$ and $L_{1}^{(2)}$ of $L_{1}^{(1)}$ are not visible in this chart. The Painlevé vector field and the anticanonical pencil both have a base point $b_{3}$ given by $u_{311}=4, u_{312}=0$.

The second coordinate chart after the third blowup is defined by

$$
\begin{aligned}
u_{211} & =u_{321} \\
u_{212} & =u_{322} u_{211} \\
u_{321} & =u_{1}{ }^{-2} u_{2}=u_{311} u_{312}, \\
u_{322} & =u_{1}{ }^{3} u_{2}{ }^{-2}=u_{311}{ }^{-1} \\
u_{1} & =u_{321}{ }^{-2} u_{322}{ }^{-1} \\
u_{2} & =u_{321}{ }^{-3} u_{322}{ }^{-2} \\
\dot{u}_{321} & =u_{322}{ }^{-1}\left(-2+6 u_{322}+u_{321}{ }^{4} u_{322}{ }^{3}+(5 z)^{-1} u_{321} u_{322}\right), \\
\dot{u}_{322} & =u_{321}{ }^{-1}\left(3-12 u_{322}-2 u_{321}{ }^{4} u_{322}{ }^{3}\right), \\
w_{32} & =u_{321}{ }^{6} u_{322}{ }^{4}, \\
{\left[w_{32}\left(1-4 u_{322}\right)^{-1}\right]^{\bullet} } & =2 u_{321}{ }^{6} u_{322}{ }^{4}\left(1-4 u_{322}\right)^{-2}\left(-u_{321}{ }^{3} u_{322}{ }^{2}+3(5 z)^{-1}\left(1-4 u_{322}\right)\right), \\
E w_{32} & =2^{-1}-2 u_{322}-u_{321}{ }^{4} u_{322}{ }^{3}, \\
\dot{E} w_{32} & =(5 z)^{-1}\left(-3+12 u_{322}+2 u_{321}{ }^{4} u_{322}{ }^{3}\right) .
\end{aligned}
$$

The equations $u_{321}=0$ and $u_{322}=0$ define $L_{3}$ and $L_{2}^{(1)}$, respectively. The lines $L_{0}^{(3)}$ and $L_{1}^{(2)}$ are not visible. The Painlevé vector field and the anticanonical pencil both have a base point $b_{3}$ given by $u_{321}=0, u_{322}=1 / 4$ in this chart. 
A.5. Resolution of the flow at $b_{3}$. Blowing up $S_{3}$ at $b_{3}$ leads to $S_{4}$. First coordinate chart:

$$
\begin{aligned}
& u_{311}-4=u_{411} u_{312}, \\
& u_{312}=u_{412} \text {, } \\
& u_{411}=u_{1}^{-4} u_{2}\left(-4 u_{1}^{3}+u_{2}^{2}\right) \text {, } \\
& u_{412}=u_{1} u_{2}{ }^{-1} \text {, } \\
& u_{1}=u_{412}{ }^{-2}\left(4+u_{411} u_{412}\right)^{-1}, \\
& u_{2}=u_{412}{ }^{-3}\left(4+u_{411} u_{412}\right)^{-1}, \\
& \dot{u}_{411}=u_{412}{ }^{-1}\left(4+u_{411} u_{412}\right)^{-1} \\
& \times\left(-10 u_{411}-4 u_{411}{ }^{2} u_{412}+128 u_{412}{ }^{3}+112 u_{411} u_{412}{ }^{4}+32 u_{411}{ }^{2} u_{412}{ }^{5}\right. \\
& \left.+3 u_{411}{ }^{3} u_{412}{ }^{6}-(5 z)^{-1} u_{411} u_{412}\left(4+u_{411} u_{412}\right)\right) \text {, } \\
& \dot{u}_{412}=\left(4+u_{411} u_{412}\right)^{-1} \\
& \times\left(-2+u_{411} u_{412}-16 u_{412}{ }^{4}-8 u_{411} u_{412}{ }^{5}-u_{411}{ }^{2} u_{412}{ }^{6}\right. \\
& \left.+(5 z)^{-1} u_{412}\left(4+u_{411} u_{412}\right)\right) \text {, } \\
& w_{41}=u_{412}{ }^{5}\left(4+u_{411} u_{412}\right)^{3}, \\
& {\left[w_{41} u_{411}{ }^{-1}\right]^{\bullet}=2 u_{412}{ }^{5}\left(4+u_{411} u_{412}\right)^{3} u_{411}{ }^{-2}\left(-u_{412}{ }^{2}\left(4+u_{411} u_{412}\right)^{2}+3(5 z)^{-1} u_{411}\right) \text {, }} \\
& E w_{41}=2^{-1} u_{411}-u_{412}{ }^{3}\left(4+u_{411} u_{412}\right)^{2} \text {, } \\
& \dot{E} w_{41}=(5 z)^{-1}\left(-3 u_{411}+2 u_{412}{ }^{3}\left(4+u_{411} u_{412}\right)^{2}\right) \text {. }
\end{aligned}
$$

Then $u_{412}=0$ defines $L_{4}$ and $4+u_{411} u_{412}=0$ defines the proper transform $L_{0}^{(4)}$ of $L_{0}^{(3)}$. The proper transforms of the other lines on which the Painlevé vector field is infinite are not visible in this chart. The Painlevé vector field and the anticanonical pencil both have a base point $b_{4}$ given by $u_{411}=0, u_{412}=0$.

The second coordinate chart after the fourth blowup is defined by

$$
\begin{aligned}
u_{311}-4 & =u_{421}, \\
u_{312} & =u_{422}\left(u_{311}-4\right), \\
u_{421} & =u_{1}{ }^{-3}\left(-4 u_{1}^{3}+u_{2}{ }^{2}\right)=u_{411} u_{412}, \\
u_{422} & =u_{1}{ }^{4} u_{2}{ }^{-1}\left(-4 u_{1}{ }^{3}+u_{2}{ }^{2}\right)^{-1}=u_{411}{ }^{-1}, \\
u_{1} & =u_{421}{ }^{-2}\left(4+u_{421}\right)^{-1} u_{422}{ }^{-2}, \\
u_{2} & =u_{421}{ }^{-3}\left(4+u_{421}\right)^{-1} u_{422}{ }^{-3}, \\
\dot{u}_{421} & =u_{422}{ }^{-1}\left(-3+32 u_{421}{ }^{3} u_{422}{ }^{4}+16 u_{421}{ }^{4} u_{422}{ }^{4}+2 u_{421}{ }^{5} u_{422}{ }^{4}\right), \\
\dot{u}_{422}= & u_{421}{ }^{-1}\left(4+u_{421}\right)^{-1}\left(10+4 u_{421}-128 u_{421}{ }^{3} u_{422}{ }^{4}-112 u_{421} u_{422}{ }^{4}\right. \\
& \left.-32 u_{421}{ }^{5} u_{422}{ }^{4}-3 u_{421}{ }^{6} u_{422}{ }^{4}+(5 z)^{-1} u_{421}\left(4+u_{421}\right) u_{422}\right), \\
w_{42}= & u_{421}{ }^{5}\left(4+u_{421}\right)^{3} u_{422}{ }^{6}, \\
\dot{w}_{42}= & 2 u_{421}{ }^{5}\left(4+u_{421}\right)^{3} u_{422}{ }^{6}\left(-u_{421}{ }^{2}\left(4+u_{421}\right)^{2} u_{422}{ }^{3}+3(5 z)^{-1}\right), \\
E w_{42}= & 2^{-1}-u_{421}{ }^{3}\left(4+u_{421}\right)^{2} u_{422}{ }^{4}, \\
\dot{E} w_{42}= & (5 z)^{-1}\left(-3+2 u_{421}{ }^{3}\left(4+u_{421}\right)^{2} u_{422}{ }^{4}\right) .
\end{aligned}
$$

The equations $u_{421}=0,4+u_{421}=0$, and $u_{422}=0$ define $L_{4}, L_{0}^{(4)}$, and and $L_{3}^{(1)}$, respectively. The proper transforms of the other lines on which the Painlevé vector field is infinite are not visible in this chart. Both the Painlevé vector field and the anticanonical pencil have no base point in this chart. 
A.6. Resolution of the flow at $b_{4}$. Blowing up $S_{4}$ at $b_{4}$ leads to $S_{5}$. First coordinate chart:

$$
\begin{aligned}
& u_{411}=u_{511} u_{412} \text {, } \\
& u_{412}=u_{512} \text {, } \\
& u_{511}=u_{2}^{2}\left(-4 u_{1}^{3}+u_{2}^{2}\right) u_{1}{ }^{-5} \text {, } \\
& u_{512}=u_{1} u_{2}{ }^{-1} \text {, } \\
& u_{1}=u_{512}{ }^{-2}\left(4+u_{511} u_{512}^{2}\right)^{-1}, \\
& u_{2}=u_{512}{ }^{-3}\left(4+u_{511} u_{512}^{2}\right)^{-1}, \\
& \dot{u}_{511}=u_{512}{ }^{-1}\left(4+u_{511} u_{512}\right)^{-1} \\
& \times\left(-8 u_{511}+128 u_{512}{ }^{2}-5 u_{511}{ }^{2} u_{512}{ }^{2}+128 u_{511} u_{512}{ }^{4}+40 u_{511}{ }^{2} u_{512}{ }^{6}\right. \\
& \left.+4 u_{511}{ }^{3} u_{512}{ }^{8}-2(5 z)^{-1} u_{511} u_{512}\left(4+u_{511} u_{512}^{2}\right)\right) \text {, } \\
& \dot{u}_{512}=\left(4+u_{511} u_{512}^{2}\right)^{-1} \\
& \times\left(-2+u_{511} u_{512}{ }^{2}-16 u_{512}{ }^{4}-8 u_{511} u_{512}{ }^{6}-u_{511}{ }^{2} u_{512}{ }^{8}\right. \\
& \left.+(5 z)^{-1} u_{512}\left(4+u_{511} u_{512}^{2}\right)\right) \text {, } \\
& w_{51}=u_{512}^{4}\left(4+u_{511} u_{512}^{2}\right)^{3}, \\
& {\left[w_{51} u_{511}{ }^{-1}\right]^{\bullet}=2 u_{512}{ }^{4}\left(4+u_{511} u_{512}{ }^{2}\right)^{3} u_{511}{ }^{-2}\left(-u_{512}\left(4+u_{511} u_{512}{ }^{2}\right)^{2}+3(5 z)^{-1} u_{511}\right) \text {, }} \\
& E w_{51}=2^{-1} u_{511}-u_{512}^{2}\left(4+u_{511} u_{512}^{2}\right)^{2}, \\
& \dot{E} w_{51}=(5 z)^{-1}\left(-3 u_{511}+2 u_{512}^{2}\left(4+u_{511} u_{512}^{2}\right)^{2}\right) \text {. }
\end{aligned}
$$

Then $u_{512}=0$ defines $L_{5}$ and $4+u_{511} u_{512}^{2}=0$ defines the proper transform $L_{0}^{(5)}$ of $L_{0}^{(4)}$. The proper transforms of the other lines on which the Painlevé vector field is infinite are not visible in this chart. The Painlevé vector field and the anticanonical pencil both have a base point $b_{5}$ given by $u_{511}=0, u_{512}=0$.

The second coordinate chart after the fifth blowup is defined by

$$
\begin{aligned}
u_{411}= & u_{521}, \\
u_{412}= & u_{522} u_{411}, \\
u_{521}= & u_{1}{ }^{-4}\left(-4 u_{1}{ }^{3}+u_{2}{ }^{2}\right) u_{2}=u_{511} u_{512}, \\
u_{522}= & u_{1}{ }^{5}\left(-4 u_{1}{ }^{3}+u_{2}{ }^{2}\right)^{-1} u_{2}{ }^{-2}=u_{511}{ }^{-1}, \\
u_{1}= & u_{521}{ }^{-2} u_{522}{ }^{-2}\left(4+u_{521}{ }^{2} u_{522}\right)^{-1}, \\
u_{2}= & u_{521}{ }^{-3} u_{522}{ }^{-3}\left(4+u_{521}{ }^{2} u_{522}\right)^{-1}, \\
\dot{u}_{521}= & u_{522}{ }^{-1}\left(4+u_{521}{ }^{2} u_{522}\right)^{-1} \\
& \times\left(-10-4 u_{521}{ }^{2} u_{522}+128 u_{521}{ }^{2} u_{522}{ }^{3}+112 u_{521}{ }^{4} u_{522}{ }^{4}+32 u_{521}{ }^{6} u_{522}{ }^{5}\right. \\
& \left.+3 u_{521}{ }^{5} u_{522}{ }^{6}-(5 z)^{-1} u_{521} u_{522}\left(4+u_{521}{ }^{2} u_{522}\right)\right), \\
& \times\left(8+5 u_{521}{ }^{2} u_{522}-128 u_{521}{ }^{2} u_{522}{ }^{3}-128 u_{521}{ }^{4} u_{522}{ }^{4}-40 u_{521}{ }^{6} u_{522}{ }^{5}\right. \\
\dot{u}_{522}= & u_{521}{ }^{-1}\left(4+u_{521}{ }^{2} u_{522}{ }^{6}+2(5 z)^{-1} u_{521} u_{522}\left(4+u_{521}{ }^{2} u_{522}\right)\right), \\
w_{52}= & u_{521}{ }^{4} u_{522}{ }^{5}\left(4+u_{521}{ }^{2} u_{522}\right)^{3}, \\
\dot{w}_{52}= & 2 u_{521}{ }^{4} u_{522}{ }^{5}\left(4+u_{521}{ }^{2} u_{522}\right)^{3}\left(-u_{521} u_{522}{ }^{2}\left(4+u_{521}{ }^{2} u_{522}\right)^{2}+3(5 z)^{-1}\right), \\
E w_{52}= & 2^{-1}-u_{521}{ }^{2} u_{522}{ }^{3}\left(4+u_{521}{ }^{2} u_{522}\right)^{2}, \\
\dot{E} w_{52}= & (5 z)^{-1}\left(-3+2 u_{521}{ }^{2} u_{522}{ }^{3}\left(4+u_{521}{ }^{2} u_{522}\right)^{2}\right) .
\end{aligned}
$$


The equations $u_{521}=0,4+u_{521}^{2} u_{522}=0$, and $u_{522}=0$ define $L_{5}, L_{0}^{(5)}$, and and $L_{4}^{(1)}$, respectively. The proper transforms of the other lines on which the Painlevé vector field is infinite are not visible in this chart. Both the Painlevé vector field and the anticanonical pencil have no base point in this chart.

A.7. Resolution of the flow at $b_{5}$. Blowing up $S_{5}$ at $b_{5}$ leads to $S_{6}$. First coordinate chart:

$$
\begin{aligned}
& u_{511}=u_{611} u_{512} \text {, } \\
& u_{512}=u_{612} \text {, } \\
& u_{611}=u_{1}{ }^{-6} u_{2}^{3}\left(-4 u_{1}^{3}+u_{2}^{2}\right), \\
& u_{612}=u_{1} u_{2}^{-1} \text {, } \\
& u_{1}=u_{612}{ }^{-2}\left(4+u_{611} u_{612}\right)^{-1}, \\
& u_{2}=u_{612}{ }^{-3}\left(4+u_{611} u_{612}\right)^{-1} \text {, } \\
& \dot{u}_{611}=u_{612}{ }^{-1}\left(4+u_{611} u_{612}{ }^{3}\right)^{-1} \\
& \times\left(-6 u_{611}+128 u_{612}-6 u_{611}{ }^{2} u_{612}{ }^{3}\right. \\
& +144 u_{611} u_{612}{ }^{4}+48 u_{611}{ }^{2} u_{612}{ }^{7}+5 u_{611}{ }^{3} u_{612}{ }^{10} \\
& \left.-3(5 z)^{-1} u_{611} u_{612}\left(4+u_{611} u_{612}{ }^{3}\right)\right), \\
& \dot{u}_{612}=\left(4+u_{611} u_{612}{ }^{3}\right)^{-1} \\
& \times\left(-2+u_{611} u_{612}{ }^{3}-16 u_{612}{ }^{4}-8 u_{611} u_{612}{ }^{7}\right. \\
& \left.\left.-u_{611}{ }^{2} u_{612}{ }^{10}+(5 z)^{-1} u_{612}\left(4+u_{611} u_{612}{ }^{3}\right)\right)\right) \\
& w_{61}=u_{612}{ }^{3}\left(4+u_{611} u_{612}{ }^{3}\right)^{3}, \\
& {\left[w_{61} u_{611}{ }^{-1}\right]^{\bullet}=2 u_{612}{ }^{3}\left(4+u_{611} u_{612}{ }^{3}\right)^{3} u_{611}{ }^{-2}} \\
& \times\left(-\left(4+u_{611} u_{612}\right)^{2}+3(5 z)^{-1} u_{611}\right), \\
& E w_{61}=2^{-1} u_{611}-u_{612}\left(4+u_{611} u_{612}\right)^{2} \text {, } \\
& \dot{E} w_{61}=(5 z)^{-1}\left(-3 u_{611}+2 u_{612}\left(4+u_{611} u_{612}{ }^{3}\right)^{2}\right) \text {. }
\end{aligned}
$$

Then $u_{612}=0$ defines $L_{6}$ and $4+u_{611} u_{612}{ }^{3}=0$ defines the proper transform $L_{0}^{(6)}$ of $L_{0}^{(5)}$. The proper transforms of the other lines on which the Painlevé vector field is infinite are not visible in this chart. The Painlevé vector field and the anticanonical pencil both have a base point $b_{6}$ given by $u_{611}=0, u_{612}=0$. 
The second coordinate chart after the sixth blowup is defined by

$$
\begin{aligned}
u_{511}= & u_{621}, \\
u_{512}= & u_{622} u_{511}, \\
u_{621}= & u_{1}{ }^{-5} u_{2}{ }^{2}\left(-4 u_{1}{ }^{3}+u_{2}{ }^{2}\right)=u_{611} u_{612}, \\
u_{622}= & u_{1}{ }^{6} u_{2}{ }^{-3}\left(-4 u_{1}{ }^{3}+u_{2}{ }^{2}\right)^{-1}=u_{611}{ }^{-1}, \\
u_{1}= & u_{621}{ }^{-2} u_{622}{ }^{-2}\left(4+u_{621}{ }^{3} u_{622}{ }^{-1},\right. \\
u_{2}= & u_{621}{ }^{-3} u_{622}{ }^{-3}\left(4+u_{621}{ }^{3} u_{622}{ }^{2}\right)^{-1} \\
\dot{u}_{621}= & u_{622}{ }^{-1}\left(4+u_{621}{ }^{3} u_{622}\right)^{-1} \\
& \times\left(-8+128 u_{621} u_{622}{ }^{2}-5 u_{621}{ }^{3} u_{622}{ }^{2}\right. \\
& +128 u_{621}{ }^{4} u_{622}{ }^{4}+40 u_{621}{ }^{7} u_{622}{ }^{6}+4 u_{621}{ }^{10} u_{622}{ }^{8} \\
& \left.-2(5 z)^{-1} u_{621} u_{622}\left(4+u_{621}{ }^{3} u_{622}{ }^{2}\right)\right), \\
\dot{u}_{622}= & u_{621}{ }^{-1}\left(4+u_{621}{ }^{3} u_{622}\right)^{-1} \\
& \times\left(6-128 u_{621} u_{622}{ }^{2}+6 u_{621}{ }^{3} u_{622}{ }^{2}\right. \\
& -144 u_{621}{ }^{4} u_{622}{ }^{4}-48 u_{621}{ }^{7} u_{622}{ }^{6}-5 u_{621}{ }^{10} u_{622}{ }^{8} \\
& \left.+3(5 z)^{-1} u_{621} u_{622}\left(4+u_{621}{ }^{3} u_{622}{ }^{2}\right)\right), \\
w_{62}= & u_{621}{ }^{3} u_{622}{ }^{4}\left(4+u_{621}{ }^{3} u_{622}{ }^{2}\right)^{3}, \\
\dot{w}_{622}= & 2 u_{621}{ }^{3} u_{622}{ }^{4}\left(4+u_{621}{ }^{3} u_{622}{ }^{2}\right)^{3} \\
& \times\left(-u_{622}\left(4+u_{621}{ }^{3} u_{622}{ }^{2}\right)^{2}+3(5 z)^{-1}\right), \\
E w_{62}= & 2^{-1}-u_{621} u_{622}{ }^{2}\left(4+u_{621}{ }^{3} u_{622}{ }^{2}\right)^{2}, \\
\dot{E}_{62}= & (5 z)^{-1}\left(-3+2 u_{621} u_{622}{ }^{2}\left(4+u_{621}{ }^{3} u_{622}{ }^{2}\right)^{2}\right) .
\end{aligned}
$$

The equations $u_{621}=0,4+u_{621}{ }^{3} u_{622}{ }^{2}=0$, and $u_{622}=0$ define $L_{6}, L_{0}^{(6)}$, and and $L_{5}^{(1)}$, respectively. The proper transforms of the other lines on which the Painlevé vector field is infinite are not visible in this chart. Both the Painlevé vector field and the anticanonical pencil have no base point in this chart. 
A.8. Resolution of the flow at $b_{6}$. Blowing up $S_{6}$ at $b_{6}$ leads to $S_{7}$. First coordinate chart:

$$
\begin{aligned}
& u_{611}=u_{711} u_{612} \text {, } \\
& u_{612}=u_{712} \text {, } \\
& u_{711}=u_{1}{ }^{-7} u_{2}^{4}\left(-4 u_{1}^{3}+u_{2}^{2}\right), \\
& u_{712}=u_{1} u_{2}{ }^{-1} \text {, } \\
& u_{1}=u_{712}{ }^{-2}\left(4+u_{711} u_{712}\right)^{-1}, \\
& u_{2}=u_{712}{ }^{-3}\left(4+u_{711} u_{712}\right)^{-1} \text {, } \\
& \dot{u}_{711}=u_{712}{ }^{-1}\left(4+u_{711} u_{712}^{4}\right)^{-1} \\
& \times\left(128-4 u_{711}+160 u_{711} u_{712}{ }^{4}-7 u_{711}{ }^{2} u_{712}{ }^{4}\right. \\
& +56 u_{711}^{2} u_{712}{ }^{8}+6 u_{711}^{3} u_{712}{ }^{12} \\
& \left.-4(5 z)^{-1} u_{711} u_{712}\left(4+u_{711} u_{712}{ }^{4}\right)\right) \text {, } \\
& \dot{u}_{712}=\left(4+u_{711} u_{712}{ }^{4}\right)^{-1} \\
& \times\left(-2-16 u_{712}{ }^{4}+u_{711} u_{712}{ }^{4}-8 u_{711} u_{712}{ }^{8}\right. \\
& \left.-u_{711}{ }^{2} u_{712}{ }^{12}+(5 z)^{-1} u_{712}\left(4+u_{711} u_{712}{ }^{4}\right)\right) \text {, } \\
& w_{71}=u_{712}^{2}\left(4+u_{711} u_{712}\right)^{3}, \\
& {\left[w_{71}\left(u_{711}-32\right)^{-1}\right]^{\bullet}=-2 u_{712}{ }^{2}\left(4+u_{711} u_{712}{ }^{4}\right)^{2}\left(u_{711}-32\right)^{-2}} \\
& \times\left(u _ { 7 1 2 } { } ^ { 3 } \left(1024-64 u_{711}+512 u_{711} u_{712}{ }^{4}+12 u_{711}{ }^{2} u_{712}{ }^{4}\right.\right. \\
& \left.+64 u_{711}^{2} u_{712}^{8}+u_{711}^{3} u_{712}^{8}\right) \\
& \left.+(5 z)^{-1}\left(32-3 u_{711}\right)\left(4+u_{711} u_{712}^{4}\right)\right), \\
& E w_{71}=2^{-1} u_{711}-\left(4+u_{711} u_{712}\right)^{2} \text {, } \\
& \dot{E} w_{71}=(5 z)^{-1}\left(-3 u_{711}+2\left(4+u_{711} u_{712}{ }^{4}\right)^{2}\right) \text {. }
\end{aligned}
$$

Then $u_{712}=0$ defines $L_{7}$ and $4+u_{711} u_{712}{ }^{4}=0$ defines the proper transform $L_{0}^{(7)}$ of $L_{0}^{(6)}$. The proper transforms of the other lines on which the Painlevé vector field is infinite are not visible in this chart. The Painlevé vector field and the anticanonical pencil both have a base point $b_{7}$ given by $u_{711}=32, u_{712}=0$. Remarkably this base point in the Boutroux coordinates does not depend on the independent variable $z$, whereas the seventh base point in the unscaled system is given by $y_{711}=32 x, y_{712}=0$.

The second coordinate chart after the seventh blowup is defined by

$$
\begin{aligned}
u_{611} & =u_{721}, \\
u_{612} & =u_{722} u_{611}, \\
u_{721} & =u_{1}{ }^{-6} u_{2}{ }^{3}\left(-4 u_{1}{ }^{3}+u_{2}^{2}\right)=u_{711} u_{712}, \\
u_{722} & =u_{1}{ }^{7} u_{2}{ }^{-4}\left(-4 u_{1}{ }^{3}+u_{2}{ }^{2}\right)^{-1}=u_{711}{ }^{-1}, \\
u_{1} & =u_{721}{ }^{-2} u_{722}{ }^{-2}\left(4+u_{721}{ }^{4} u_{722}{ }^{3}\right)^{-1}, \\
u_{2} & =u_{721}{ }^{-3} u_{722}{ }^{-3}\left(4+u_{721}{ }^{4} u_{722}{ }^{3}\right)^{-1}
\end{aligned}
$$




$$
\begin{aligned}
\dot{u}_{721}= & u_{722}{ }^{-1}\left(4+u_{721}{ }^{4} u_{722}{ }^{3}\right)^{-1} \\
& \times\left(-6+128 u_{722}-6 u_{721}{ }^{4} u_{722}{ }^{3}+144 u_{721}{ }^{4} u_{722}{ }^{4}+48 u_{721}{ }^{8} u_{722}{ }^{7}\right. \\
& \left.+5 u_{721}{ }^{12} u_{722}{ }^{10}-3(5 z)^{-1} u_{721} u_{722}\left(4+u_{721}{ }^{4} u_{722}{ }^{3}\right)\right), \\
\dot{u}_{722}= & -u_{721}{ }^{-1}\left(4+u_{721}{ }^{4} u_{722}{ }^{3}\right)^{-1} \\
& \times\left(4-128 u_{722}+7 u_{721}{ }^{4} u_{722}{ }^{3}-160 u_{721}{ }^{4} u_{722}{ }^{4}-56 u_{721}{ }^{8} u_{722}{ }^{7}\right. \\
& \left.-6 u_{721}{ }^{12} u_{722}{ }^{10}+4(5 z)^{-1} u_{721} u_{722}\left(4+u_{721}{ }^{4} u_{722}{ }^{3}\right)\right), \\
w_{72}= & u_{721}{ }^{2} u_{722}{ }^{3}\left(4+u_{721}{ }^{4} u_{722}{ }^{3}\right)^{3}, \\
{\left[w_{72}\left(32 u_{722}-1\right)^{-1}\right]^{\bullet}=} & 2 u_{721}{ }^{2} u_{722}{ }^{3}\left(4+u_{721}{ }^{4} u_{722}{ }^{2}\right)^{2}\left(32 u_{722}-1\right)^{-2} \\
& \times\left(u _ { 7 2 1 } { } ^ { 3 } u _ { 7 2 2 } { } ^ { 3 } \left(-64+1024 u_{722}+12 u_{721}{ }^{4} u_{722}{ }^{3}\right.\right. \\
& \left.+512 u_{721}{ }^{4} u_{722}{ }^{4}+u_{721}{ }^{8} u_{722}{ }^{6}+64 u_{721}{ }^{8} u_{722}{ }^{7}\right) \\
& \left.+(5 z)^{-1}\left(-3+32 u_{722}\right)\left(4+u_{721}{ }^{4} u_{722}{ }^{3}\right)\right), \\
E w_{72}= & 2^{-1}-u_{722}\left(4+u_{721}{ }^{4} u_{722}{ }^{3}\right)^{2}, \\
\dot{E} w_{72}= & (5 z)^{-1}\left(-3+2 u_{722}\left(4+u_{721}{ }^{4} u_{722}{ }^{3}\right)^{2}\right) .
\end{aligned}
$$

The equations $u_{721}=0,4+u_{721}{ }^{4} u_{722}{ }^{3}=0$, and $u_{722}=0$ define $L_{7}, L_{0}^{(7)}$, and and $L_{6}^{(1)}$, respectively. The proper transforms of the other lines on which the Painlevé vector field is infinite are not visible in this chart. The Painlevé vector field and the anticanonical pencil have the base point $u_{721}=0$, $u_{722}=1 / 32$ in this chart, which is equal to the previously found base point $b_{7}$.

A.9. Resolution of the flow at $b_{7}$. Blowing up $S_{7}$ at $b_{7}$ leads to $S_{8}$. First coordinate chart:

$$
\begin{aligned}
& u_{711}-32=u_{811} u_{712}, \\
& u_{712}=u_{812} \text {, } \\
& u_{811}=-u_{1}{ }^{-8} u_{2}\left(32 u_{1}^{7}+4 u_{1}^{3} u_{2}^{4}-u_{2}{ }^{6}\right), \\
& u_{812}=u_{1} u_{2}^{-1} \text {, } \\
& u_{1}=u_{812}{ }^{-2}\left(4+32 u_{812}{ }^{4}+u_{811} u_{812}{ }^{5}\right)^{-1}, \\
& u_{2}=u_{812}{ }^{-3}\left(4+32 u_{812}{ }^{4}+u_{811} u_{812}{ }^{5}\right)^{-1}, \\
& \dot{u}_{811}=u_{812}{ }^{-1}\left(4+32 u_{812}{ }^{4}+u_{811} u_{812}{ }^{5}\right)^{-1} \\
& \times\left[-2\left(u_{811}+1024 u_{812}{ }^{3}+152 u_{811} u_{812}{ }^{4}+4 u_{811}{ }^{2} u_{812}{ }^{5}\right)\right. \\
& +u_{812}{ }^{7}\left(32+u_{811} u_{812}\right)\left(1792+64 u_{811} u_{812}+6144 u_{812}{ }^{4}+416 u_{811} u_{812}{ }^{5}\right. \\
& \left.\left.+7 u_{811}{ }^{2} u_{812}{ }^{6}\right)-(5 z)^{-1}\left(128+5 u_{811} u_{812}\right)\left(4+32 u_{812}{ }^{4}+u_{811} u_{812}{ }^{5}\right)\right] \text {, } \\
& \dot{u}_{812}=-\left(4+32 u_{812}{ }^{4}+u_{811} u_{812}\right)^{-1} \\
& \times\left[2-16 u_{812}{ }^{4}-u_{811} u_{812}{ }^{5}+256 u_{812}{ }^{8}+8 u_{811} u_{812}{ }^{9}+1024 u_{812}{ }^{12}\right. \\
& \left.+64 u_{811} u_{812}{ }^{13}+u_{811}{ }^{2} u_{812}{ }^{14}-(5 z)^{-1} u_{812}\left(4+u_{811} u_{812}{ }^{5}+32 u_{812}{ }^{9}\right)\right] \text {, } \\
& w_{81}=u_{812}\left(4+32 u_{812}{ }^{4}+u_{811} u_{812}{ }^{5}\right)^{3}, \\
& E w_{81}=2^{-1} u_{811}-u_{812}{ }^{3}\left(32+u_{811} u_{812}\right)\left(8+32 u_{812}{ }^{4}+u_{811} u_{812}{ }^{5}\right) \text {, } \\
& \dot{E} w_{81}=(5 z)^{-1} u_{812}{ }^{-1}\left(-64-3 u_{811} u_{812}\right. \\
& \left.+2 u_{812}^{4}\left(32+u_{811} u_{812}\right)\left(8+32 u_{812}{ }^{4}+u_{811} u_{812}{ }^{5}\right)\right) \text {. }
\end{aligned}
$$


Furthermore

$$
\begin{aligned}
& {\left[w_{81}\left(u_{811}+256(5 z)^{-1}\right)^{-1}\right]^{\bullet}=2 u_{812}\left(4+32 u_{812}{ }^{4}+u_{811} u_{812}{ }^{5}\right)^{2}\left(u_{811}+256(5 z)^{-1}\right)^{-2}} \\
& \times\left[-u_{812}{ }^{2}\left(-2^{10}-2^{6} u_{811} u_{812}+2^{12} \cdot 7 u_{812}{ }^{4}+2^{8} \cdot 5 u_{811} u_{812}{ }^{5}+2^{2} \cdot 3 u_{811}{ }^{2} u_{812}{ }^{6}\right.\right. \\
& \left.+2^{15} \cdot 3 u_{812}{ }^{8}+2^{10} \cdot 7 u_{811} u_{812}{ }^{9}+2^{5} \cdot 5 u_{811}{ }^{2} u_{812}{ }^{10}+u_{811}{ }^{3} u_{812}{ }^{11}\right) \\
& +(5 z)^{-1}\left(2^{2} \cdot 3 u_{811}-2^{12} \cdot 5 u_{812^{3}} 2^{5} \cdot 3^{3} u_{811} u_{812}{ }^{4}+3 u_{811}{ }^{2} u_{812}{ }^{5}+2^{15} \cdot 5 u_{812}{ }^{7}\right. \\
& \left.+2^{10} \cdot 5 u_{811} u_{812}{ }^{8}+2^{17} \cdot 5 u_{812}{ }^{11}+2^{13} \cdot 5 u_{811} u_{812}{ }^{12}+2^{7} \cdot 5 u_{811}{ }^{2} u_{812}{ }^{13}\right) \\
& \left.+768(5 z)^{-2}\left(4+32 u_{812}{ }^{4}+u_{811} u_{812}{ }^{5}\right)\right] .
\end{aligned}
$$

The equation $u_{812}=0$ defines $L_{8}$ and $4+32 u_{812}{ }^{4}+u_{811} u_{812}{ }^{5}=0$ defines the proper transform $L_{0}^{(8)}$ of $L_{0}^{(7)}$. The proper transforms of the other lines on which the Painlevé vector field is infinite are not visible in this chart. The Painlevé vector field has a base point $b_{8}$ given by $u_{811}=-256(5 z)^{-1}$, $u_{812}=0$. In the Boutroux coordinates, this is the first base point which depends on the independent variable $z$. The anticanonical pencil has a base point $b_{8}^{\text {ell }}$ given by $u_{811}=, u_{812}=0$. We have $b_{8}^{\text {ell }} \neq b_{8}$ with a distance between both base point vanishing of order $1 / z$ as $z \rightarrow \infty$.

The second coordinate chart after the eighth blowup is defined by

$$
\begin{aligned}
& u_{711}-32=u_{821}, \\
& u_{712}=u_{822}\left(u_{711}-32\right) \text {, } \\
& u_{821}=-u_{1}{ }^{-7}\left(32 u_{1}{ }^{7}+4 u_{1}{ }^{3} u_{2}{ }^{4}-u_{2}{ }^{6}\right)=u_{811} u_{812} \text {, } \\
& u_{822}=-u_{1}{ }^{8} u_{2}{ }^{-1}\left(32 u_{1}{ }^{7}+4 u_{1}{ }^{3} u_{2}{ }^{4}-u_{2}{ }^{6}\right)^{-1}=u_{811}{ }^{-1} \text {, } \\
& u_{1}=u_{821}{ }^{-2} u_{822}{ }^{-2}\left(4+32 u_{821}{ }^{4} u_{822}{ }^{4}+u_{821}{ }^{5} u_{822}{ }^{4}\right)^{-1} \text {, } \\
& u_{2}=u_{821}{ }^{-3} u_{822}{ }^{-3}\left(4+32 u_{821}{ }^{4} u_{822}{ }^{4}+u_{821}{ }^{5} u_{822}{ }^{4}\right)^{-1}, \\
& \dot{u}_{821}=u_{822}{ }^{-1}\left(4+32 u_{821}{ }^{4} u_{822}{ }^{4}+u_{821}{ }^{5} u_{822}{ }^{4}\right)^{-1} \\
& \times\left[-4-2048 u_{821}{ }^{3} u_{822}{ }^{4}-288 u_{821}{ }^{4} u_{822}{ }^{4}-7 u_{821}{ }^{5} u_{822}{ }^{4}\right. \\
& +2 u_{821}{ }^{7}\left(32+u_{821}\right)^{2} u_{822}{ }^{8}\left(28+96 u_{821}{ }^{4} u_{822}{ }^{4}+3 u_{821}{ }^{5} u_{822}{ }^{4}\right) \\
& \left.-4(5 z)^{-1}\left(32+u_{821}\right) u_{822}\left(4+32 u_{821}{ }^{4} u_{822}{ }^{4}+u_{821}{ }^{5} u_{822}{ }^{4}\right)\right] \text {, } \\
& \dot{u}_{822}=-u_{821}{ }^{-1}\left(4+32 u_{821}{ }^{4} u_{822}{ }^{4}+u_{821}{ }^{5} u_{822}{ }^{4}\right)^{-1} \\
& \times\left[-2-8 u_{821}{ }^{3}\left(256+38 u_{821}+u_{821}^{2}\right) u_{822}{ }^{4}\right. \\
& +u_{821}{ }^{7}\left(32+u_{821}\right) u_{822}{ }^{8}\left(1792+64 u_{821}+6144 u_{821}{ }^{4} u_{822}{ }^{4}+416 u_{821}{ }^{5} u_{822}{ }^{4}\right. \\
& \left.\left.+7 u_{821}{ }^{6} u_{822}{ }^{4}\right)-(5 z)^{-1}\left(128+5 u_{821}\right) u_{822}\left(4+32 u_{821}{ }^{4} u_{822}{ }^{4}+u_{821}{ }^{5} u_{822}{ }^{4}\right)\right] \text {, } \\
& w_{82}=u_{821} u_{822}{ }^{2}\left(4+32 u_{821}{ }^{4} u_{822}{ }^{4}+u_{821}{ }^{5} u_{822}{ }^{4}\right)^{3}, \\
& E w_{82}=2^{-1}-u_{821}{ }^{3}\left(32+u_{821}\right) u_{822}{ }^{4}\left(8+32 u_{821}{ }^{4} u_{822}{ }^{4}+u_{821}{ }^{5} u_{822}{ }^{4}\right) \text {, } \\
& \dot{E} w_{82}=(5 z)^{-1} u_{821}{ }^{-1}\left(-64-3 u_{821}\right. \\
& \left.+2 u_{821}{ }^{4}\left(32+u_{821}\right) u_{822}{ }^{4}\left(8+32 u_{821}{ }^{4} u_{822}{ }^{4}+u_{821}{ }^{5} u_{822}{ }^{4}\right)\right) \text {. }
\end{aligned}
$$


Furthermore,

$$
\begin{aligned}
& {\left[w_{82}\left(256(5 z)^{-1} u_{822}+1\right)^{-1}\right]^{\bullet}=} \\
& 2 u_{822}{ }^{2}\left(4+32 u_{821}{ }^{4} u_{822}{ }^{4}+u_{821}{ }^{5} u_{822}{ }^{4}\right)^{2}\left(256(5 z)^{-1} u_{822}+1\right)^{-2} \\
& \times\left[-u_{821}{ }^{3} u_{822}{ }^{3}\left(-2^{10}-2^{6} u_{821}+2^{17} \cdot 7 u_{821}{ }^{4} u_{822}{ }^{4}+2^{8} \cdot 5 u_{821}{ }^{5} u_{822}{ }^{4}+2^{2} \cdot 3 u_{821}{ }^{6} u_{822}{ }^{4}\right.\right. \\
& \left.+2^{15} \cdot 3 u_{821}{ }^{8} u_{822}{ }^{8}+2^{10} \cdot 7 u_{821}{ }^{9} u_{822}{ }^{8}+2^{5} \cdot 5 u_{821}{ }^{10} u_{822}{ }^{8}+u_{821}{ }^{11} u_{822}{ }^{8}\right) \\
& -(5 z)^{-1}\left(-2^{8}-2^{2} \cdot 3 u_{821}-2^{18} u_{821}{ }^{3} u_{822}{ }^{4}-2^{11} \cdot 3^{2} u_{821}{ }^{4} u_{822}{ }^{4}\right. \\
& -2^{5} \cdot 5 u_{821}{ }^{5} u_{822}{ }^{4}-3 u_{821}{ }^{6} u_{822}{ }^{4}+2^{20} \cdot 7 u_{821}{ }^{7} u_{822}{ }^{8} \\
& +2^{16} \cdot 5 u_{821}{ }^{8} u_{822}{ }^{8}+2^{10} \cdot 3 u_{821}{ }^{9} u_{822}{ }^{8}+2^{23} \cdot 3 u_{821}{ }^{11} u_{822}{ }^{12} \\
& \left.+2^{18} \cdot 7 u_{821}{ }^{12} u_{822}{ }^{12}+2^{13} \cdot 5 u_{821}{ }^{13} u_{822}{ }^{12}+2^{8} u_{821}{ }^{14} u_{822}{ }^{12}\right) \\
& \left.+2^{7}(5 z)^{-2}\left(2^{7}+11 u_{821}\right)\left(4+32 u_{821}{ }^{4} u_{822}{ }^{4}+u_{821}{ }^{5} u_{822}{ }^{4}\right) u_{822}\right] .
\end{aligned}
$$

The equations $u_{821}=0,4+32 u_{821}{ }^{4} u_{822}{ }^{4}+u_{821}{ }^{5} u_{822}{ }^{4}=0$, and $u_{822}=0$ define $L_{8}, L_{0}^{(8)}$, and and $L_{7}^{(1)}$, respectively. The proper transforms of the other lines on which the Painlevé vector field is infinite are not visible in this chart. The Painlevé vector field has the base point $b_{8}$ defined by the equations $u_{821}=0,256(5 z)^{-1} u_{822}+1=0$. The base point $b_{8}^{\text {ell }}$ of the anticanonical pencil is not visible in this chart.

A.10. Resolution of the flow at $b_{8}$. Blowing up $S_{8}$ at $b_{8}=b_{8}(z)$ leads to $S_{9}=S_{9}(z)$. First coordinate chart:

$$
\begin{aligned}
& u_{811}+256(5 z)^{-1}=u_{911} u_{812}, \\
& u_{812}=u_{912} \text {, } \\
& u_{911}=u_{1}^{-9} u_{2}\left(-32 u_{1}^{7} u_{2}-4 u_{1}^{3} u_{2}^{5}+u_{2}^{7}+256(5 z)^{-1} u_{1}^{8}\right) \text {, } \\
& u_{912}=u_{1} u_{2}^{-1} \text {, } \\
& u_{1}=u_{912}{ }^{-2}\left(4+32 u_{912}{ }^{4}+u_{911} u_{912}{ }^{6}-256(5 z)^{-1} u_{912}\right)^{-1} \text {, } \\
& u_{2}=u_{912}{ }^{-3}\left(4+32 u_{912}{ }^{4}+u_{911} u_{912}{ }^{6}-256(5 z)^{-1} u_{912}\right)^{-1} \text {, } \\
& \dot{u}_{911}=\left(4+32 u_{912}{ }^{4}+u_{911} u_{912}{ }^{6}-256(5 z)^{-1} u_{912}{ }^{5}\right)^{-1} \\
& \times\left[u _ { 9 1 2 } \left(-2^{11}-2^{6} \cdot 5 u_{911} u_{912}{ }^{2}+2^{13} \cdot 7 u_{912}{ }^{4}-3^{2} u_{911}{ }^{2} u_{912}{ }^{4}\right.\right. \\
& +2^{12} u_{911} u_{912}{ }^{6}+2^{16} \cdot 3 u_{912}{ }^{8}+2^{3} \cdot 3^{2} u_{911}{ }^{2} u_{912}{ }^{8} \\
& \left.+2^{12} \cdot 5 u_{911} u_{912}{ }^{10}+2^{6} \cdot 11 u_{911^{2}} u_{912}{ }^{12}+2^{3} u_{911^{3}} u_{912}{ }^{14}\right) \\
& -2(5 z)^{-1}\left(2^{2} \cdot 3 u_{911}-2^{12} \cdot 3^{2} u_{912}{ }^{2}-2^{5} \cdot 3^{2} \cdot 7 u_{911} u_{912}{ }^{4}\right. \\
& +2^{15} \cdot 3 \cdot 5 u_{912}{ }^{6}+3 u_{911}{ }^{2} u_{912}{ }^{6}+2^{10} \cdot 17 u_{911} u_{912}{ }^{8} \\
& \left.+2^{17} \cdot 19 u_{912}{ }^{10}+2^{13} \cdot 3 \cdot 7 u_{911} u_{912}{ }^{12}+2^{7} \cdot 23 u_{911}{ }^{2} u_{912}{ }^{14}\right) \\
& +2^{9}(5 z)^{-2} u_{912}{ }^{3}\left(-2^{6} \cdot 3 \cdot 5+3 u_{911} u_{912}{ }^{2}\right. \\
& \left.+2^{13} u_{912}{ }^{4}+2^{14} \cdot 5 u_{912}{ }^{8}+2^{8} \cdot 11 u_{911} u_{912}{ }^{10}\right) \\
& \left.-2^{24} \cdot 7(5 z)^{-3} u_{912}{ }^{12}\right]
\end{aligned}
$$




$$
\begin{aligned}
& \dot{u}_{912}=-\left(4+32 u_{912}{ }^{4}+u_{911} u_{912}{ }^{6}-256(5 z)^{-1} u_{912}\right)^{-1} \\
& \times\left[2-2^{4} u_{912}{ }^{4}-u_{911} u_{912}{ }^{6}+2^{8} u_{912}{ }^{8}+2^{3} u_{911} u_{912}{ }^{10}\right. \\
& +2^{10} u_{912}{ }^{12}+2^{6} u_{911} u_{912}{ }^{14}+u_{911}{ }^{2} u_{912}{ }^{16} \\
& -(5 z)^{-1} u_{912}\left(2^{2}-2^{5} \cdot 7 u_{912}{ }^{4}+u_{911} u_{912}{ }^{6}\right. \\
& \left.+2^{11} u_{912}{ }^{8}+2^{14} u_{912}{ }^{12}+2^{9} u_{911} u_{912}{ }^{14}\right) \\
& \left.+2^{8}(5 z)^{-2} u_{912}{ }^{6}\left(1+2^{8} u_{912}{ }^{8}\right)\right] \text {, } \\
& w_{91}=\left(4+32 u_{912}{ }^{4}+u_{911} u_{912}{ }^{6}-256(5 z)^{-1} u_{912}{ }^{5}\right)^{3}, \\
& \left.\dot{w}_{91}=3 u_{912}{ }^{3}\left(4+32 u_{912}{ }^{4}+u_{911} u_{912}{ }^{6}-256(5 z)^{-1} u_{912}{ }^{5}\right)^{2}\right) \\
& \times\left[-2^{6}-3 u_{911} u_{912}{ }^{2}+2^{9} u_{912}{ }^{4}+2^{4} u_{911} u_{912}{ }^{6}+2^{11} u_{912}{ }^{8}\right. \\
& +2^{7} u_{911} u_{912}{ }^{10}+2 u_{911}{ }^{2} u_{912}{ }^{12} \\
& -2^{8}(5 z)^{-1} u_{912}\left(-3+2^{4} u_{912}{ }^{4}+2^{7} u_{912}{ }^{8}+2^{2} u_{911} u_{912}{ }^{10}\right) \\
& \left.+2^{17}(5 z)^{-2} u_{912}{ }^{10}\right] \text {, } \\
& E w_{91}=-2^{-1} u_{912}{ }^{-1}\left[u _ { 9 1 2 } \left(-u_{911}+2^{9} u_{912}{ }^{2}+2^{4} u_{911} u_{912}{ }^{4}\right.\right. \\
& \left.+2^{11} u_{912}{ }^{6}+2^{7} u_{911} u_{912}{ }^{8}+2 u_{911}{ }^{2} u_{912}{ }^{10}\right) \\
& -2^{8}(5 z)^{-1}\left(-1+2^{4} u_{912}{ }^{4}+2^{7} u_{912}{ }^{8}+4 u_{911} u_{912}{ }^{10}\right) \\
& \left.+2^{17}(5 z)^{-2} u_{912}{ }^{9}\right] \text {, } \\
& \dot{E} w_{91}=(5 z)^{-1} u_{912}{ }^{-2}\left[-2^{6}-3 u_{911} u_{912}{ }^{2}+2^{9} u_{912}{ }^{4}+2^{4} u_{911} u_{912}{ }^{6}\right. \\
& +2^{11} u_{912}{ }^{8}+2^{7} u_{911} u_{912}{ }^{10}+2 u_{911}{ }^{2} u_{912}{ }^{12} \\
& -2^{8}(5 z)^{-1} u_{912}\left(-3+2^{4} u_{912}{ }^{4}+2^{7} u_{912}{ }^{8}+2^{2} u_{911} u_{912}{ }^{10}\right) \\
& \left.2^{17}(5 z)^{-2} u_{912}{ }^{10}\right] \text {. }
\end{aligned}
$$

As the change of coordinates from $\left(u_{1}, u_{2}\right)$ to all previous coordinate systems $\left(u_{i j 1}, u_{i j 2}\right)$ for $i \leq 8$ do not depend on $z$ the limiting system of differential equations ${ }^{0} \dot{u}_{1}=u_{2},{ }^{0} \dot{u}_{2}=6 u_{1}{ }^{2}+1$ in the coordinate systems $\left(u_{i j 1}, u_{i j 2}\right)$ for $i \leq 8$ is obtained by deleting the term in $\dot{u}_{i j 1}$ and $\dot{u}_{i j 2}$ which have a factor $1 / z$ in front. This is no longer true in the coordinate system $\left(u_{911}, u_{912}\right)$. However, the difference between the Painlevé-Boutroux system and the limiting system still has a relatively simple expression:

$$
\begin{array}{ccc}
\dot{u}_{911}-{ }^{0} \dot{u}_{911}= & -2(5 z)^{-1} u_{912}{ }^{-2}\left(64-640(5 z)^{-1} u_{912}+3 u_{911} u_{912}{ }^{2}\right) \\
\dot{u}_{912}-{ }^{0} \dot{u}_{912}= & (5 z)^{-1} u_{912} .
\end{array}
$$

The equation $u_{912}=0$ defines $L_{9}$ and $4+32 u_{912}{ }^{4}+u_{911} u_{912}{ }^{6}-256(5 z)^{-1} u_{912}{ }^{5}=0$ defines the proper transform $L_{0}^{(9)}$ of $L_{0}^{(8)}$. The proper transforms of the other lines on which the Painlevé vector field is infinite are not visible in this chart. The Painlevé vector field is regular along $L_{9}$, nonzero, and transversal to it. Moreover, the Painleveé vector field has no base points in this chart. On the other hand the blowing up of $S_{8}$ in the point $b_{8}$, which is not the base point of the anticanonical pencil, causes $E w_{91}$ to be infinite along $L_{9}$, the line determined by the equation $u_{912}=0$. The image ${ }^{(1)} b_{8}^{\text {ell }}$ of $b_{8}^{\text {ell }}$ in $S_{9}$ is not visible in this coordinate chart. 
The second coordinate chart after the ninth blowup is defined by

$$
\begin{aligned}
& u_{921}=u_{811}+2^{8}(5 z)^{-1}, \\
& u_{812}=u_{922}\left(u_{811}+2^{8}(5 z)^{-1}\right) \text {, } \\
& u_{921}=u_{1}^{-8}\left(-2^{5} u_{1}^{7} u_{2}-2^{2} u_{1}^{3} u_{2}^{5}+u_{2}^{7}+2^{8}(5 z)^{-1} u_{1}^{8}\right)=u_{911} u_{912} \text {, } \\
& u_{922}=u_{1}{ }^{9} u_{2}{ }^{-1}\left(-2^{5} u_{1}{ }^{7} u_{2}-2^{2} u_{1}{ }^{3} u_{2}{ }^{5}+u_{2}{ }^{7}+2^{8}(5 z)^{-1} u_{1}^{8}\right)^{-1}=u_{911}{ }^{-1} \text {, } \\
& u_{1}=u_{921}{ }^{-2} u_{922}{ }^{-2}\left(2^{2}+2^{5} u_{921}{ }^{4} u_{922}{ }^{4}+u_{921}{ }^{6} u_{922}{ }^{5}-2^{8}(5 z)^{-1} u_{921}{ }^{5} u_{922}{ }^{5}\right)^{-1} \text {, } \\
& u_{2}=u_{921}{ }^{-3} u_{922}{ }^{-3}\left(2^{2}+2^{5} u_{921}{ }^{4} u_{922}{ }^{4}+u_{921}{ }^{6} u_{922}{ }^{5}-2^{8}(5 z)^{-1} u_{921}{ }^{5} u_{922}{ }^{5}\right)^{-1} \text {, } \\
& \dot{u}_{921}=-u_{922}{ }^{-1}\left(2^{2}+2^{5} u_{921}{ }^{4} u_{922}{ }^{4}+u_{921}{ }^{6} u_{922}{ }^{5}-2^{8}(5 z)^{-1} u_{921}{ }^{5} u_{922}{ }^{5}\right)^{-1} \\
& \times\left[2+2^{11} u_{921}{ }^{2} u_{922}{ }^{3}+2^{4} \cdot 19 u_{921}{ }^{4} u_{922}{ }^{4}+2^{3} u_{921}{ }^{6} u_{922}{ }^{5}\right. \\
& -2^{13} \cdot 7 u_{921}{ }^{6} u_{922}{ }^{7}-2^{8} \cdot 3 \cdot 5 u_{921}{ }^{8} u_{922}{ }^{8}-2^{6} u_{921}{ }^{10} u_{922}{ }^{9} \\
& -2^{16} \cdot 3 u_{921}{ }^{10} u_{922}{ }^{11}-2^{10} \cdot 19 u_{921}{ }^{12} u_{922}{ }^{12}-2^{7} \cdot 5 u_{921}{ }^{14} u_{922}{ }^{13} \\
& -7 u_{921}{ }^{16} u_{922}{ }^{14}+(5 z)^{-1} u_{921} u_{922} \\
& \times\left(2^{2} \cdot 5-2^{13} 3^{2} u_{921}{ }^{2} u_{922}{ }^{3}-2^{5} \cdot 7 \cdot 17 u_{921}{ }^{4} u_{922}{ }^{4}\right. \\
& +5 u_{921}{ }^{6} u_{922}{ }^{5}+2^{16} \cdot 3 \cdot 5 u_{921}{ }^{6} u_{922}{ }^{7}+2^{15} u_{921}{ }^{8} u_{922}{ }^{8} \\
& \left.+2^{18} \cdot 19 u_{921}{ }^{10} u_{922}{ }^{11}+2^{16} \cdot 5 u_{921}{ }^{12} u_{922}{ }^{12}+2^{8} \cdot 3 \cdot 7 u_{921}{ }^{14} u_{922}{ }^{13}\right) \\
& -2^{8}(5 z)^{-2} u_{921}{ }^{4} u_{922}{ }^{5}\left(-2^{7} \cdot 3 \cdot 5+5 u_{921}{ }^{2} u_{922}\right. \\
& \left.+2^{14} u_{921}{ }^{4} u_{922}{ }^{4}+2^{15} \cdot 5 u_{921}{ }^{8} u_{922}{ }^{8}+2^{8} \cdot 3 \cdot 7 u_{921}{ }^{10} u_{922}{ }^{9}\right) \\
& \left.2^{24} \cdot 7(5 z)^{-3} u_{921}{ }^{13} u_{922}{ }^{14}\right] \text {, } \\
& \dot{u}_{922}=-\left(2^{2}+2^{5} u_{921}{ }^{4} u_{922}{ }^{4}+u_{921}{ }^{6} u_{922}{ }^{5}-2^{8}(5 z)^{-1} u_{921}{ }^{5} u_{922}{ }^{5}\right)^{-1} u_{922} \\
& \times\left[u _ { 9 2 1 } u _ { 9 2 2 } { } ^ { 2 } \left(-2^{11}-2^{6} \cdot 5 u_{921}{ }^{2} u_{922}-3^{2} u_{921}{ }^{4} u_{922}{ }^{2}\right.\right. \\
& +2^{13} \cdot 7 u_{921}{ }^{4} u_{922}{ }^{4}+2^{12} u_{921}{ }^{6} u_{922}{ }^{5}+2^{3} \cdot 3^{2} u_{921}{ }^{8} u_{922}{ }^{6} \\
& +2^{16} \cdot 3 u_{921}{ }^{8} u_{922}{ }^{8}+2^{12} \cdot 5 u_{921}{ }^{10} u_{922}{ }^{9}+2^{6} \cdot 11 u_{921}{ }^{12} u_{922}{ }^{10} \\
& \left.+2^{3} u_{921}{ }^{14} u_{922}{ }^{11}\right)-2(5 z)^{-1}\left(2^{2} \cdot 3-2^{12} \cdot 3^{2} u_{921}{ }^{2} u_{922}{ }^{3}\right. \\
& -2^{5} \cdot 3^{2} \cdot 7 u_{921}{ }^{4} u_{922}{ }^{4}+3 u_{921}{ }^{6} u_{922}{ }^{5}+2^{15} \cdot 3 \cdot 5 u_{921}{ }^{6} u_{922}{ }^{7} \\
& +2^{10} \cdot 17 u_{921}{ }^{8} u_{922}{ }^{8}+2^{17} \cdot 19 u_{921}{ }^{10} u_{922}{ }^{11}+2^{13} \cdot 3 \cdot 7 u_{921}{ }^{12} u_{922}{ }^{12} \\
& \left.+2^{7} \cdot 23 u_{921}{ }^{14} u_{922}{ }^{13}\right)+2^{9}(5 z)^{-2} u_{921}{ }^{3} u_{922}{ }^{4}\left(-2^{6} \cdot 3 \cdot 5+3 u_{921}{ }^{2} u_{922}\right. \\
& \left.+2^{13} u_{921}{ }^{4} u_{922}{ }^{4}+2^{14} \cdot 5 u_{921^{8}} u_{922}{ }^{8}+2^{8} \cdot 11 u_{921}{ }^{10} u_{922}{ }^{9}\right) \\
& \left.-2^{24} \cdot 7(5 z)^{-3} u_{921}{ }^{12} u_{922}{ }^{13}\right] \\
& w_{92}=u_{922}\left(2^{2}+2^{5} u_{921}{ }^{4} u_{922}{ }^{4}+u_{921}{ }^{6} u_{922}{ }^{5}-2^{8}(5 z)^{-1} u_{921}{ }^{5} u_{922}{ }^{5}\right)^{3} \text {, } \\
& \dot{w}_{92}=2 u_{922}\left(2^{2}+2^{5} u_{921}{ }^{4} u_{922}{ }^{4}+u_{921}{ }^{6} u_{922}{ }^{5}-2^{8}(5 z)^{-1} u_{921}{ }^{5} u_{922}{ }^{5}\right)^{2} \\
& \times\left[-u_{921} u_{922}{ }^{2}\left(-2^{10}-2^{6} u_{921}{ }^{2} u_{922}+2^{12} \cdot 7 u_{921}{ }^{4} u_{922}{ }^{4}\right.\right. \\
& +2^{8} \cdot 5 u_{921}{ }^{6} u_{922}{ }^{5}+2^{2} \cdot 3 u_{921}{ }^{8} u_{922}{ }^{6}+2^{15} \cdot 3 u_{921}{ }^{8} u_{922}{ }^{8} \\
& \left.+2^{10} \cdot 7 u_{921}{ }^{10} u_{922}{ }^{9}+2^{5} \cdot 5 u_{921}{ }^{12} u_{922}{ }^{10}+u_{921}{ }^{14} u_{922}{ }^{11}\right) \\
& +(5 z)^{-1}\left(2^{2} \cdot 3-2^{12} \cdot 3^{2} u_{921}{ }^{2} u_{922}{ }^{3}-2^{5} \cdot 3^{3} u_{921}{ }^{4} u_{922}{ }^{4}\right. \\
& +3 u_{921}{ }^{6} u_{922}{ }^{5}+2^{15} \cdot 3 \cdot 5 u_{921}{ }^{6} u_{922}{ }^{7}+2^{10} \cdot 11 u_{921}{ }^{8} u_{922}{ }^{8} \\
& \left.+2^{17} \cdot 19 u_{921}{ }^{10} u_{922}{ }^{11}+2^{13} \cdot 3 \cdot 5 u_{921}{ }^{12} u_{922}{ }^{12}+2^{7} \cdot 11 u_{921}{ }^{14} u_{922}{ }^{13}\right) \\
& -2^{8}(5 z)^{-2} u_{921}{ }^{3} u_{922}{ }^{4}\left(-2^{6} \cdot 3 \cdot 5+3 u_{921}{ }^{2} u_{922}+2^{13} u_{921}{ }^{4} u_{922}{ }^{4}\right. \\
& \left.\left.+2^{14} \cdot 5 u_{921}{ }^{8} u_{922}{ }^{8}+2^{11} u_{921}{ }^{10} u_{922}{ }^{9}\right)+2^{23} \cdot 7(5 z)^{-3} u_{921}{ }^{12} u_{922}{ }^{13}\right]
\end{aligned}
$$




$$
\begin{aligned}
E w_{92}= & -2^{-1} u_{921}{ }^{-1}\left[u _ { 9 2 1 } \left(-1+2^{9} u_{921}{ }^{2} u_{922}{ }^{3}+2^{4} u_{921}{ }^{4} u_{922}{ }^{4}\right.\right. \\
& \left.+2^{11} u_{921}{ }^{6} u_{922}{ }^{7}+2^{7} u_{921}{ }^{8} u_{922}{ }^{8}+2 u_{921}{ }^{10} u_{922}{ }^{9}\right) \\
& -2^{8}(5 z)^{-1}\left(-1+2^{4} u_{921}{ }^{4} u_{922}{ }^{4}+2^{7} u_{921}{ }^{8} u_{922}{ }^{8}+2^{2} u_{921}{ }^{10} u_{922}{ }^{9}\right) \\
& \left.+2^{17}(5 z)^{-2} u_{921}{ }^{9} u_{922}\right], \\
\dot{E} w_{92}= & (5 z)^{-1} u_{921}{ }^{-2} u_{922}{ }^{-1} \\
& \times\left[-2^{6}-3 u_{921}{ }^{2} u_{922}+2^{9} u_{921}{ }^{4} u_{922}{ }^{4}+2^{4} u_{921}{ }^{6} u_{922}{ }^{5}\right. \\
& +2^{11} u_{921}{ }^{8} u_{922}{ }^{8}+2^{7} u_{921}{ }^{10} u_{922}{ }^{9}+2 u_{921}{ }^{12} u_{922}{ }^{10} \\
& -2^{8}(5 z)^{-1} u_{921} u_{922}\left(-3+2^{4} u_{921}{ }^{4} u_{922}{ }^{4}+2^{7} u_{921}{ }^{8} u_{922}{ }^{8}\right. \\
& \left.\left.+2^{2} u_{921}{ }^{10} u_{922}{ }^{9}\right)+2^{17}(5 z)^{-2} u_{921}{ }^{10} u_{922}{ }^{10}\right] .
\end{aligned}
$$

The difference between the system and the limiting system is given by

$$
\begin{aligned}
& \dot{u}_{921}-{ }^{0} \dot{u}_{921}=-(5 z)^{-1} u_{921}{ }^{-1} u_{922}{ }^{-1}\left(128-1280(5 z)^{-1} u_{921} u_{922}+5 u_{921}{ }^{2} u_{922}\right) \\
& \dot{u}_{922}-^{0} \dot{u}_{922}=2(5 z)^{-1} u_{921}{ }^{-2}\left(64-640(5 z)^{-1} u_{921} u_{922}+3 u_{921}{ }^{2} u_{922}\right) .
\end{aligned}
$$

The equations $u_{921}=0,2^{2}+2^{5} u_{921}{ }^{4} u_{922}{ }^{4}+u_{921}{ }^{6} u_{922}{ }^{5}-2^{8}(5 z)^{-1} u_{921}{ }^{5} u_{922}{ }^{5}=0$, and $u_{922}=0$ define $L_{9}, L_{0}^{(9)}$, and and $L_{8}^{(1)}$, respectively. The proper transforms of the other lines on which the Painlevé vector field is infinite are not visible in this chart.

\section{REFERENCES}

[1] D.V. Anosov: Geodesic Flows on Closed Riemann Manifolds with Negative Curvature. English translation of the Proceedings of the Steklov Institute of mathematics, No. 90, 1967. American Mathematical Society, Providence, Rhode Island, 1969.

[2] P. Boutroux: Recherches sur les transcendantes de M. Painlevé et l'étude asymptotique des équations différentielles du second ordre. Ann. Sci. École Norm. Sup., Série 330 (1913) 255-375. (suite) (3) 31 (1914) 99-159.

[3] H. Bruns: Ueber die Perioden der elliptischen Integrale erster und zweiter Gattung. Festschrift, Dorpat, 1875. Reprinted in Mathematische Annalen 27 (1886) 234-252.

[4] E.A. Coddington and N. Levinson: Theory of Ordinary Differential Equations. McGraw-Hill, New York, etc., 1955.

[5] O. Costin: On Borel summation and Stokes phenomena for rank one nonlinear systems of ODE's. Duke Math. J. 93 (1998) 289-344.

[6] O. Costin: Correlation between pole location and asymptotic behavior for Painlevé I solutions. Comm. Pure Appl. Math. 52 (1999) 461-478.

[7] O. Costin and R.D. Costin: On the formation of singularities of solutions of nonlinear differential systems in antistokes directions. Invent. math. 145 (2001) 425-485.

[8] E. Cotton: Sur les solutions asymptotiques des équations différentielles. Ann. Sci. École Norm. Sup., Série 328 (1911) $473-521$.

[9] B. Dubrovin, T. Grava, and C. Klein: On universality of critical behavior in the focusing nonlinear Schrödinger equation, elliptic umbilic catastrophe and the tritronquée solution to the Painlevé-I equation. J. Nonlinear Sci. 19 (2009) 57-94.

[10] J.J. Duistermaat: Okamoto's space for the Painlevé equation $P_{I}$. Preprint, May 2009.

[11] R. Gérard et A.H.M. Levelt: Sur les connexions à singularités régulières dans le cas de plusieurs variables. Funkcialaj Ekvacioj 19 (1976) 149-173.

[12] J. Hadamard: Sur l'itération et les solutions asymptotiques des équations différentielles. Bull. Soc. Math.France 29 (1901) $224-228$.

[13] P. Holmes and D. Spence: On a Painlev-type boundary value problem. Quart. J. Mech. Appl. Math. 37 (1984), 525-538.

[14] N. Joshi and A.V. Kitaev: On Boutroux's tritronquée solutions of the first Painlevé equation. Studies in Applied Mathematics 107 (2001) 253-291.

[15] N. Joshi and M.D. Kruskal: An asymptotic approach to the connection problem for the first and the second Painlevé equations. Physics Letters A 130 (1988) 129-137.

[16] N. Joshi and M.D. Kruskal: The Painlevé connection problem: an asymptotic approach. I. Studies in Applied Mathematics 86 (1992) 315-376.

[17] K. Kajiwara, T. Masuda, M. Noumi, Y. Ohta, and Y. Yamada: Cubic pencils and Painlevé Hamiltonians. Funkcialaj Ekvacioj 48 (2005) 147-160.

[18] A.V. Kitaev: Elliptic asymptotics of the first and second Painlevé transcendents. Uspekhi Mat. Nauk 49 (1994) $77-140$.

[19] A.A. Kapaev: Quasi-linear Stokes phenomenon for the Painlevé first equation. J. Phys. A 37 (2004) 11149-11167.

[20] B. Malgrange: Sur le théorème de Maillet. Asymptotic Anal. 2 (1989) 1-4. 
[21] K. Okamoto: Sur les feuilletages associés aux équation du second ordre à points critiques fixes de P. Painlevé. Espaces de conditions initiales. Japanese J. Math. 5 (1979) 1-79.

[22] O. Perron: Über die Stabilität und asymptotisches Verhalten der Integrale von Differentialgleichungensystemen. Math. Zeitshcr. 29 (1928) 129-160.

[23] U. Persson: Configurations of Kodaira fibers on rational elliptic surfaces. Math. Z. 205 (1990) 1-47.

$[24]$ U. Schmickler-Hirzebruch: Elliptische Flächen über $\mathbb{P}_{1}(\mathbb{C})$ mit drei Ausnahmefasern und die hypergeometrische Differentialgleichung. Schriftenreihe des Mathematischen Instituts der Universität Münster, No. 33, 1985.

[25] T. Shioda and K. Takano: On some Hamiltonian structures of Painlevé systems, I. Funkcialaj Ekvacioj 40 (1997) $271-291$.

[26] G.G. Stokes: On the discontinuity of arbitrary constants which appear in divergent developments. Trans. Cambridge Phil. Soc. 10 (1857) 106-128 = pp. 77-109 in Mathematical and Physical Papers by late sir George Gabriel Stokes, vol. 4. Cambridge University Press, 1904.

School of Mathematics and Statistics F07, The University of Sydney, NSW 2006, Australia, Tel: +61 29351 2172, FAX: +61293514534

E-mail address: nalini.joshi@sydney.edu.au 


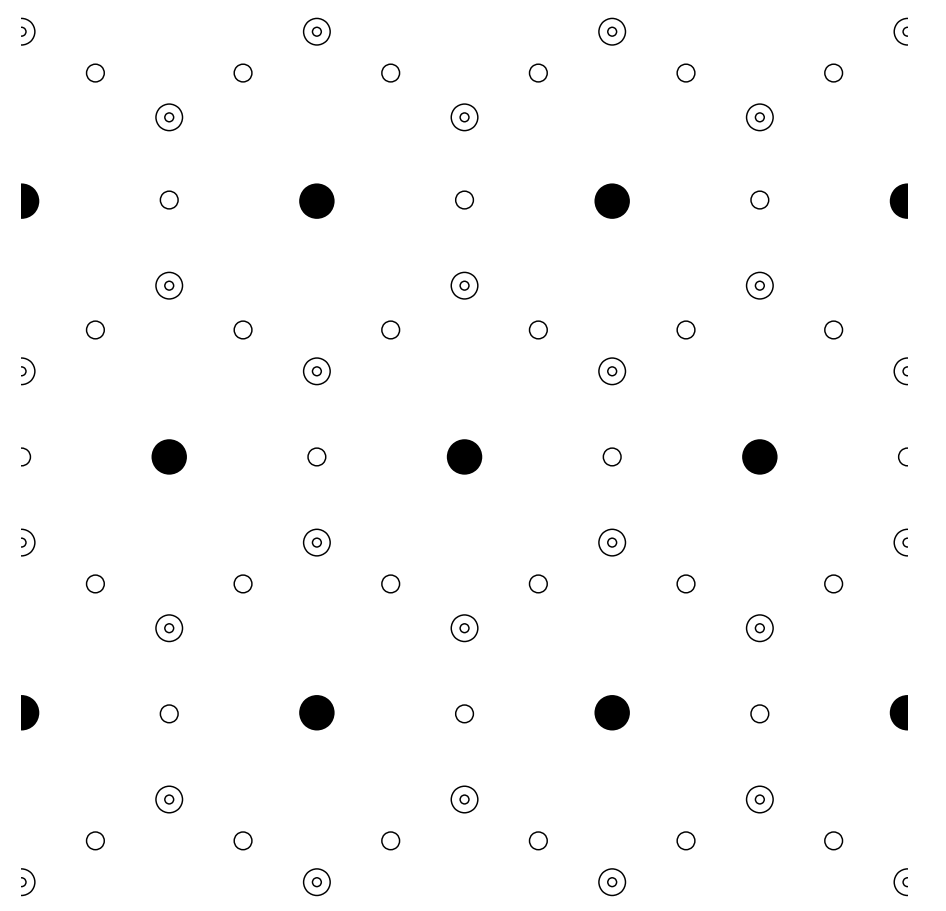

Figure 4.1. Near the components of the infinity set

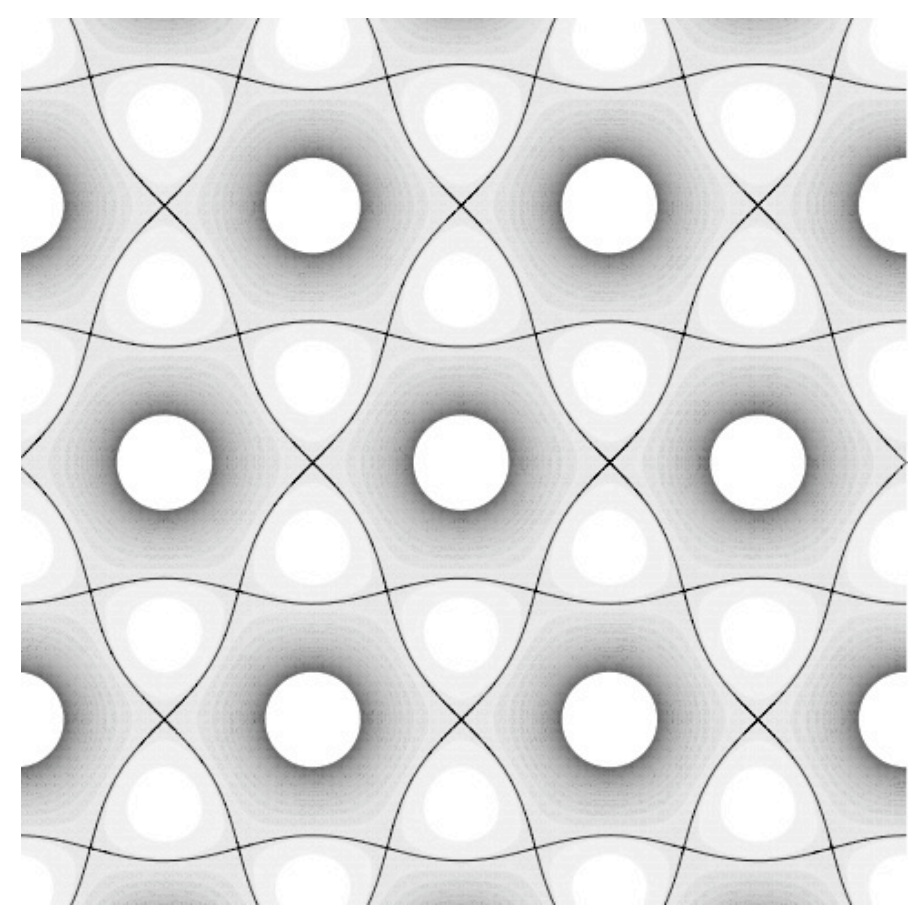

Figure 4.2. The absolute value of the Weierstrass $\wp$ function 


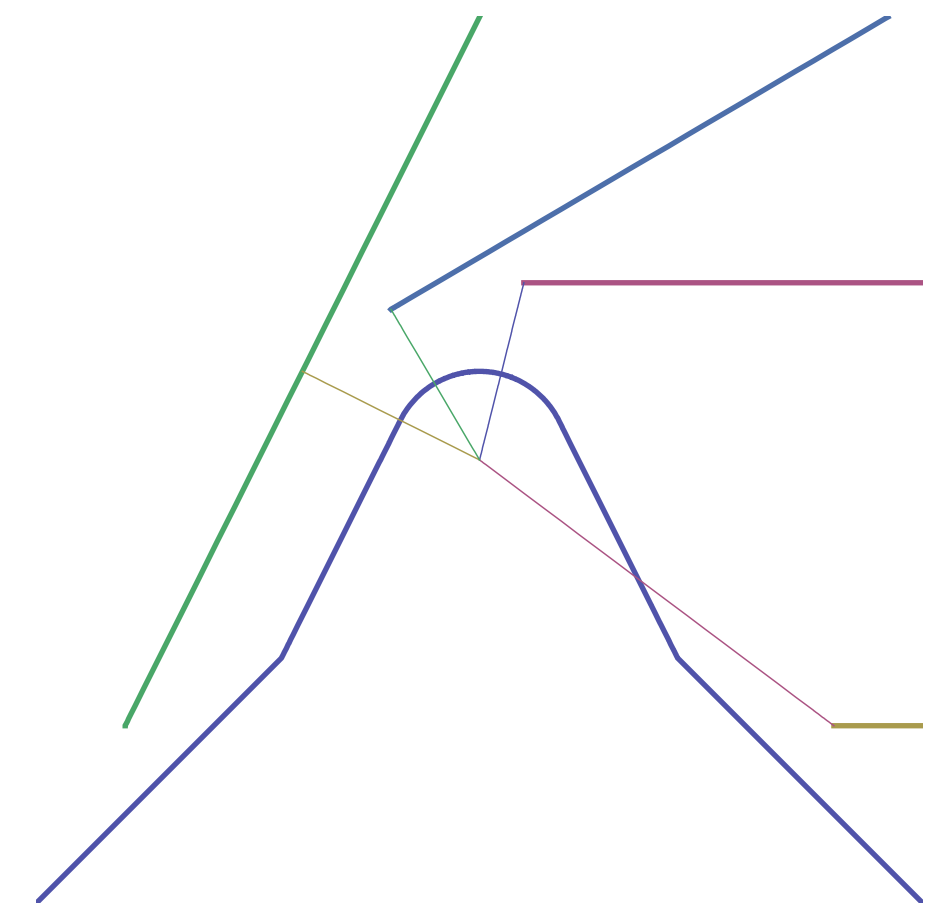

Figure 5.1. The domain $V$, four half-lines $L_{t}^{+} \subset V$, and their distances to the origin 


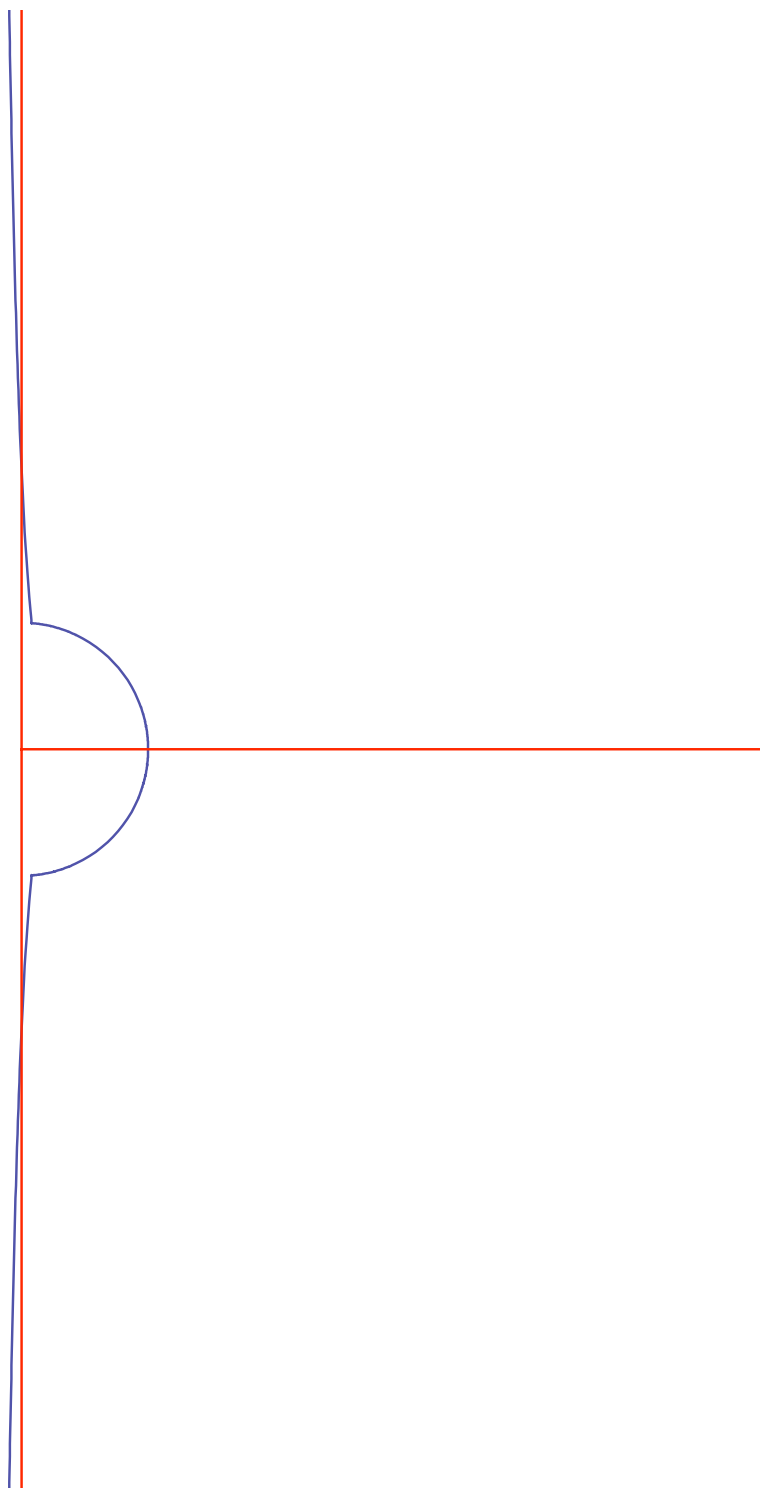

FIgURE 5.2. The region $R_{\eta, r}$, to the right of the curved boundary 


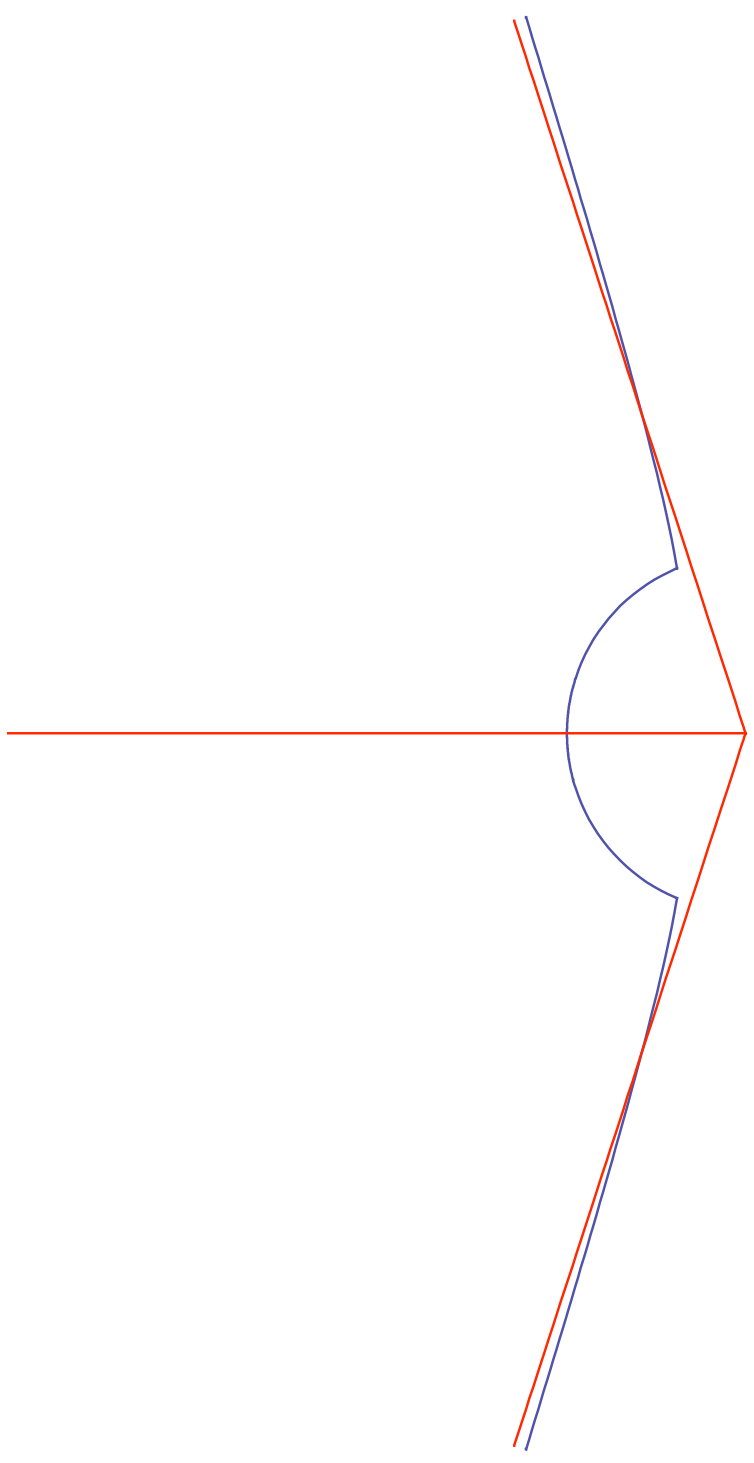

Figure 5.3. Truncated region in the $x$-plane, to the left of the curved boundary 


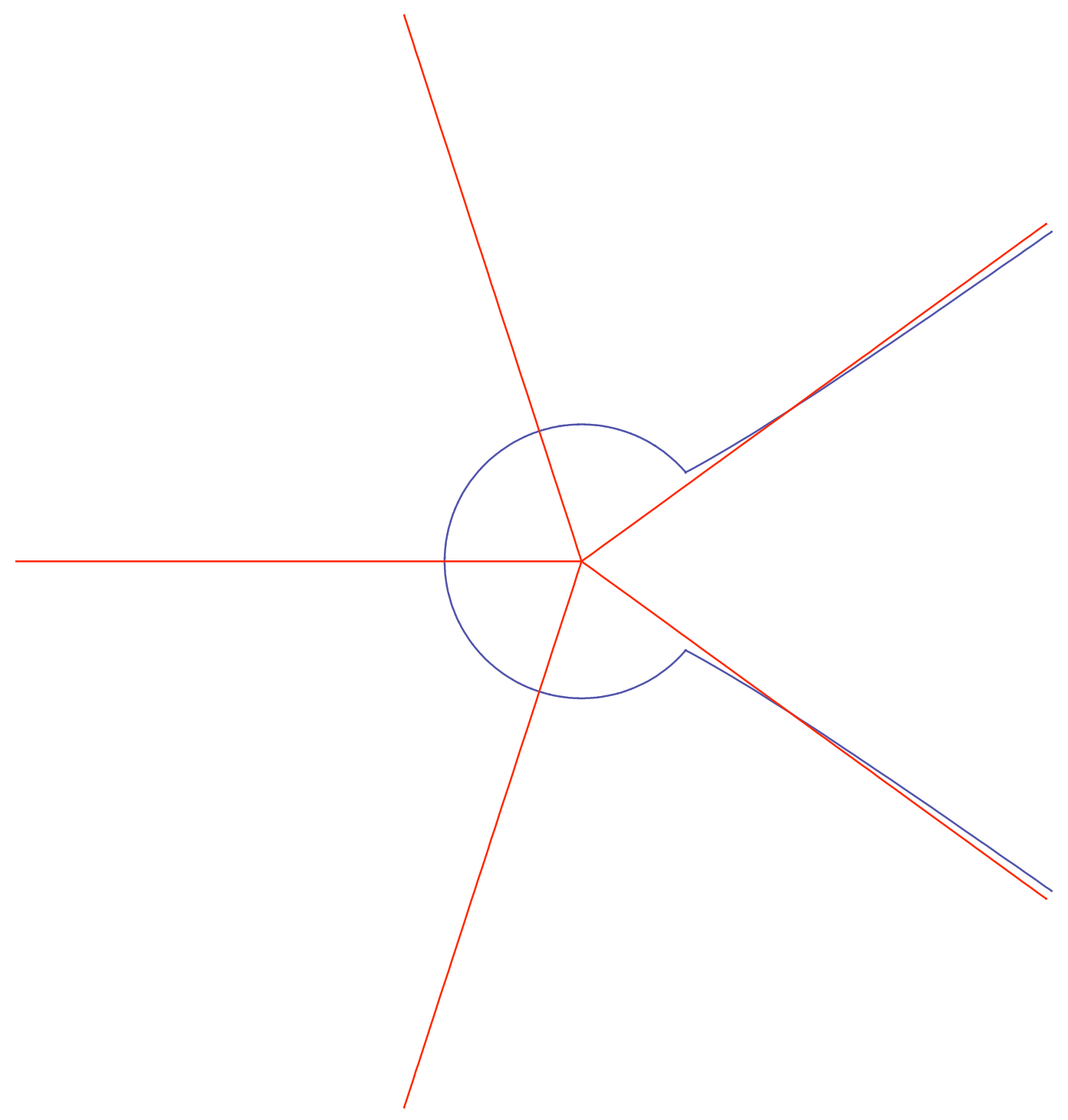

FiguRE 5.4. Triply truncated region in the $x$-plane, to the left of the curved boundary 


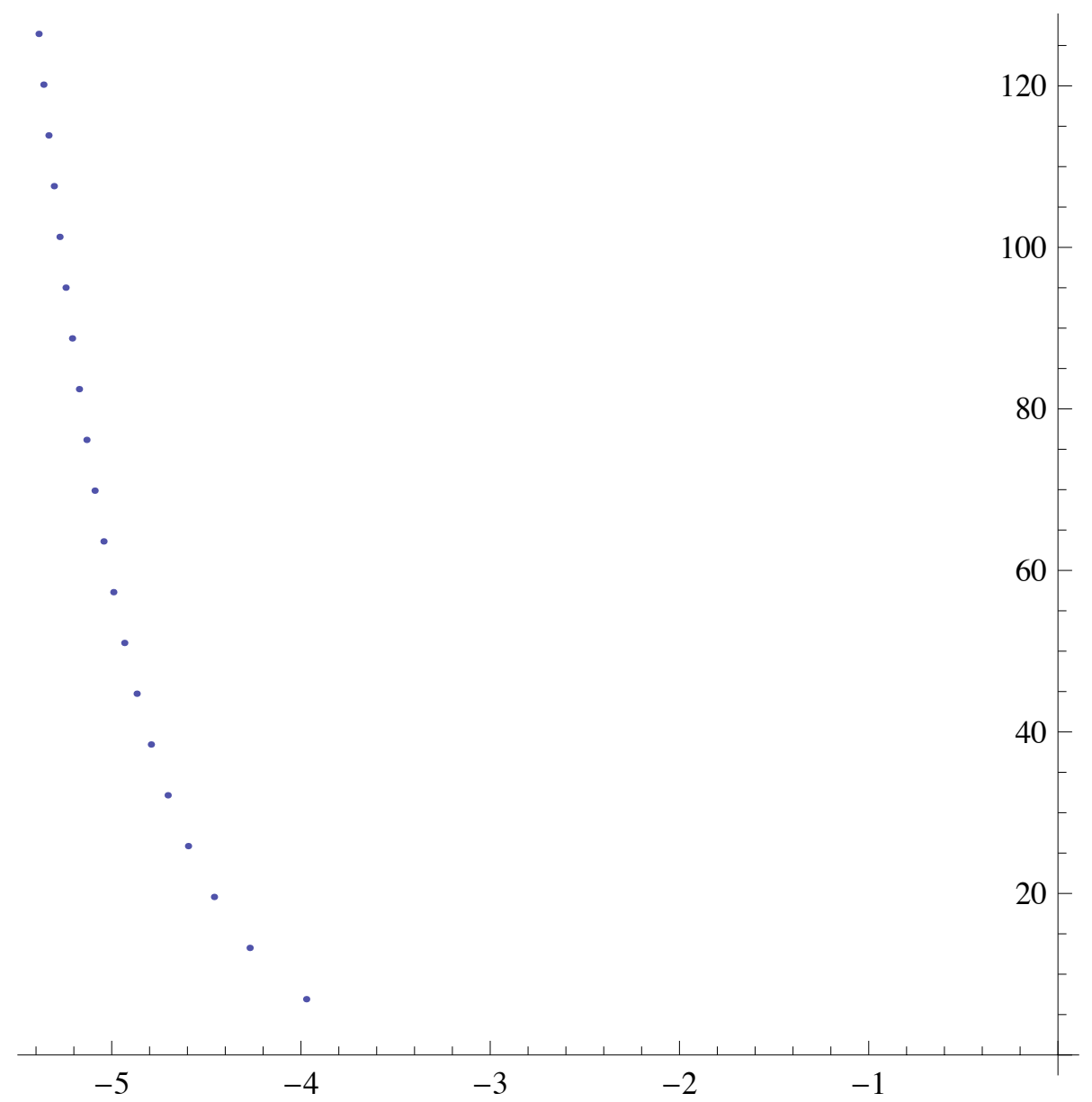

FiguRE 5.5. The first twenty of the asymptotic approximations in 5.68) of the poles of $p_{\downarrow}(t)$ 